\title{
Difusão rotacional em membrana e anisotropia de fluorescência.
}

\author{
Jozismar Rodrigues Alves
}

Dissertação apresentada ao Instituto de Física para a obtenção do título de Mestre em Ciências.

Orientadora: Profa. Dra. Vera Bohomoletz Henriques

Banca Examinadora:

Profa. Dra. Vera Bohomoletz Henriques (IFUSP)

Prof. Dr. Amando Siuiti Ito (FFCLRP)

Prof. Dr. Eduardo Fontes Henriques (UFPEL)

São Paulo

2012 


\section{FICHA CARTALOGRÁFICA}

PREPARADA PELO SERVIÇO DE BIBLIOTECA E INFORMAÇÕES DO INSTITUTO DE FÍSICA DA UNIVERSIDADE DE SÃO PAULO

Alves, Jozismar Rodrigues

Difusão rotacional em membrana e anisotropia de fluorescência. São Paulo, 2012

Dissertação (Mestrado) - Universidade de São Paulo. Instituto de Física - Depto. de Física Geral

Orientador: Profa. Dra. Vera Bohomoletz Henqriques

Área de Concentração: Física da Matéria Condensada

Unitermos: 1.Fluídos complexos;2.Física computacional; 3.Fenômeno de difusão de massa.

USP/IF/SBI-101/2012 


\section{Agradecimentos}

Agradeço,

À minha mãe Dalva Rodrigues, meu irmão Wiliam Rodrigues e ao meu grande amigo Antonio Francisco de Paula Filho (Tico), pelo amor, por sempre acreditarem na minha capacidade e me incentivar.

À professora Vera B. Henriques, pela orientação neste trabalho, pela confiança depositada, pelo acolhimento, carinho e amizade.

À minha companheira Edna de Oliveira Telles, pelo amor, carinho, paciência e compreensão pela minha ausência em certos momentos, principalmente nas etapas finais do trabalho.

Aos meus companheiros de grupo, Wagner Gomes Rodrigues Junior e Henrique Guidi, que me ajudaram durante todo o trabalho. Em especial ao Wagner, que em uma, das muitas conversas sobre meu trabalho, ajudou a elucidar muitas dúvidas.

Aos meus amigos Tiago Mendes de Almeida, Graziella Bento e Marcello Magri Amaral e todos que me acompanharam desde a graduação.

Ao meu amigo Oscar A. Barbosa, pela troca cultural e pela ajuda em algumas dúvidas de programação.

Ao Instituto de Física da USP, pela oportunidade e por disponibilizar a estrutura para a execução desse trabalho. À agência de fomento, CAPES, pelo apoio financeiro através da bolsa a mim concedida e a todos que participaram, direta ou indiretamente da minha formação. 


\section{Resumo}

Propriedades estruturais de membranas modelo, tais como as vesículas lipídicas, podem ser investigadas através da adição de sondas fluorescentes. Após sua incorporação aos sistemas biomoleculares, as moléculas fluorescentes são excitadas por luz linearmente polarizada e a emissão de fluorescência é despolarizada, devido à difusão translacional e também à difusão rotacional do fluoróforo, durante o tempo de vida do estado excitado. O monitoramento da luz emitida é feito através da técnica de fluorescência resolvida no tempo, em que são acompanhadas, por um lado, a intensidade da luz emitida, que fornece o tempo de decaimento da fluorescência, dependente do meio, e, por outro, o decaimento da anisotropia nas componentes da luz emitida, cujos tempos de correlação rotacional informam sobre a fluidez do meio. Uma molécula muito usada como sonda fluorescente é o DPH. Este fluoróforo, bastante hidrofóbico, possui simetria molecular uniaxial, e seus momentos de transição de absorção e emissão são colineares. Um dos usos desta sonda é no acompanhamento da fluidez da bicamada lipídica ao longo da transição de fase das cadeias.

A interpretação de dados experimentais requer modelos para a localização das moléculas fluorescentes, bem como para as possíveis restrições a seu movimento. Neste estudo, apresentamos três modelos para a difusão de moléculas fluorescentes uniaxiais, como o DPH, sugeridos em diversos artigos da literatura. O primeiro modelo consiste de um dipolo com rotação livre aleatória em solução homogênea, e serve como base para os modelos de difusão em meios anisotrópicos. No segundo modelo, consideramos rotações aleatórias dos dipolos reemissores no interior de cones distribuídos com seus eixos perpendiculares à vesícula esférica, geometria que pode refletir o movimento da sonda com eixo em média paralelo às cadeias lipídicas, restrito por elas. No terceiro modelo, o dipolo rotacional no plano da bicamada de geometria esférica, movimento que poderia ocorrer entre as monocamadas que constituem a bicamada. 
Para cada um dos modelos propostos analisamos a difusão rotacional da sonda através de dois métodos: (i) resolvemos a equação de difusão, para uma única molécula, levando em conta as condições de contorno impostas pelos modelos e determinamos a probabilidade da molécula ser encontrada com uma dada configuração em um tempo $t$. Considerando a distribuição de moléculas na geometria proposta, obtemos a expressão analítica para a anisotropia de fluorescência. No caso da geometria do cone, a solução é obtida numericamente; (ii) realizamos simulações numéricas do passeio aleatório rotacional restrito, nas geometrias correspondentes, método muito útil no caso de geometrias de baixa simetria ou de composição de geometrias.

Realizamos um estudo exploratório dos dados experimentais para DPH em membrana modelo, à luz dos resultados para os dois modelos propostos para este meio anisotrópico. 


\section{Abstract}

Structural properties of model membranes, such as lipid vesicles, may be investigated through the addition of fluorescent probes. After incorporation into the biomolecular system, the fluorescent molecules are excited with linearly polarized light and the fluorescence emission is depolarized due to translational as well as rotational diffusion during the lifetime of the excited state. The monitoring of emitted light is made using the technique of time-resolved fluorescence: the intensity of the emitted light emitted informs on fluorescence decay times, and the decay of the components of the emitted light yield rotational correlation times which inform on the fluidity of the medium. The fluorescent molecule DPH, of uniaxial symmetry, is rather hydrophobic and has collinear transition and emission moments. It has been used frequently as a probe for the monitoring of the fluidity of the lipid bilayer along the phase transition of the chains.

The interpretation of experimental data requires models for localization of fluorescent molecules as well as for possible restrictions on their movement. In this study, we develop calculations for three models for uniaxial diffusion of fluorescent molecules, such as DPH, suggested in several articles in the literature. The first model consists of a free randomly rotating dipole in a homogeneous solution, and serves as the basis for the study of the diffusion of models in anisotropic media. In the second model, we consider random rotations of emitting dipoles distributed within cones with their axes perpendicular to the vesicle spherical geometry: this could mimic the movement of the probe axis parallel to an average lipid chain. In the third model, the dipole rotates in the plane of the of bilayer spherical geometry, within a movement that might occur between the monolayers forming the bilayer.

For each of the models proposed, two methods are used by us in order to analyse the rotational diffusion: (I) solution of the corresponding rotational diffusion equation for a sin- 
gle molecule, taking into account the boundary conditions imposed by the models, for the probability of the fluorescent molecule to be found with a given configuration at time t. Considering the distribution of molecules in the geometry proposed, we obtain the analytical expression for the fluorescence anisotropy, except for the cone geometry, for which the solution is obtained numerically; (II) numerical simulations of a rotational random walk restricted in geometries corresponding method very useful in case of low-symmetry geometries or geometries of composition. In this study, we have reproduced existing calculations, in some cases, extended calculations developed previously by other authors, in others, or, still, developed new calculations.

As an application of our methodology for the analysis of diffusional rotations, we have conducted an exploratory study of the experimental data for DPH in lipid bilayers, in the light of the results for the two models proposed for anisotropic media. 


\section{Sumário}

1 Introdução $\quad 1$

2 Anisotropia de fluorescência $\quad 5$

3 Modelos para difusão rotacional $\quad 13$

3.1 Rotação livre . . . . . . . . . . . . . . . . . . . . . 15

3.2 Rotação paralela à superfície da membrana . . . . . . . . . . . . . . . . . 16

3.3 Rotação restrita no cone . . . . . . . . . . . . . . . . . . 17

4 Difusão rotacional e anisotropia de fluorescência - cálculo analítico $\quad 19$

4.1 A equação da difusão rotacional - 3D . . . . . . . . . . . . . . . . . . 19

4.2 A equação da difusão rotacional - 2D . . . . . . . . . . . . . . . . . 23

4.3 A função densidade probabilidade $G(\Omega, t) \ldots \ldots \ldots$. . . . . . . . 25

4.3 .1 Rotação livre . . . . . . . . . . . . . . . . 25

4.3 .2 Rotação no plano . . . . . . . . . . . . . . . . . . . . 27

4.3 .3 Difusão rotacional no cone . . . . . . . . . . . . . . . . . . 28

4.4 Excitação do dipolo e anisotropia da fluorescência . . . . . . . . . . . . . . . 32

4.5 Fluorescência de luz polarizada e anisotropia . . . . . . . . . . . . . . 35

4.5.1 Rotação livre . . . . . . . . . . . . . . . . . . 36

4.5.2 Rotação no plano . . . . . . . . . . . . . . . . . . . 38

4.5 .3 Rotação no cone . . . . . . . . . . . . . . . . . . . . . . 40

4.6 Resultados da solução numérica . . . . . . . . . . . . . . . . . . . . 45

5 Difusão rotacional e anisotropia de fluorescência - simulações numéricas 51

5.1 Rotação livre . . . . . . . . . . . . . . . . . . . . 51 
5.1 .1 Distribuição inicial . . . . . . . . . . . . . . . . 52

5.1 .2 Passeio aleatório . . . . . . . . . . . . . . . . . 54

5.1 .3 Resultados . . . . . . . . . . . . . . . . . . 56

5.2 Rotação restrita . . . . . . . . . . . . . . . . . . . 61

5.2.1 Distribuição inicial dos vetores dipolos . . . . . . . . . . . . . 62

5.2 .2 Difusão . . . . . . . . . . . . . . . . . 66

5.2 .3 Resultados ........................... 68

$\begin{array}{lll}6 & \text { Conclusão } & 79\end{array}$

$\begin{array}{ll}\text { A Parâmetros } & 81\end{array}$

A.1 Rotação livre . . . . . . . . . . . . . . . . . . . . . 81

A.1.1 Ângulo polar médio . . . . . . . . . . . . . . . . . . . . . . 81

A.1.2 Ângulo polar médio quadrático . . . . . . . . . . . . . . . 82

A.1.3 Dispersão . . . . . . . . . . . . . . . . . . . . . 83

B Coeficientes simulação numérica $\quad 85$

$\begin{array}{ll}\text { C Matriz de rotação } & 91\end{array}$

D Anisotropia do Wobbling-cone $\quad 95$

$\begin{array}{llr}\text { E } & \text { Trigonometria esférica } & 99\end{array}$

$\begin{array}{ll}\text { Referências Bibliográficas } & 105\end{array}$ 


\section{Capítulo 1}

\section{Introdução}

Estudos do comportamento da bicamada lipídica na presença de outras moléculas, da localização de proteínas na bicamada, de estrutura da bicamada em diferentes fases termodinâmicas e da restrição de movimento de sondas fluorescentes na bicamada podem ser feitas através de técnicas experimentais de fluorescência, que podem ser divididas em estáticas ou resolvidas no tempo. A escolha entre fluorescência estática e resolvida no tempo é feita de acordo com os parâmetros desejados.

Na fluorescência estática, as medidas são feitas com iluminação e observação constante, ou seja, a amostra é iluminada com luz contínua e a intensidade é medida [1]. Esse tipo de medida pode, por exemplo, fornecer a localização da sonda fluorescente na membrana, indicar se ela está em um meio mais, ou menos, hidrofóbico, através do deslocamento do espectro de emissão. Ao passo que na fluorescência resolvida no tempo, a amostra é exposta a um pulso de luz, cuja duração é menor que o tempo de decaimento da amostra. Esse tipo de medida pode fornecer o coeficiente de difusão, o tempo de decaimento da fluorescência e o tempo de correlação rotacional ou translacional da sonda fluorescente incorporada na membrana, através da anisotropia de fluorescência. Uma vez estabelecidos os parâmetros relacionados à sonda, é possível obter informações sobre o comportamento da membrana.

Em nosso trabalho, iremos considerar apenas a fluorescência resolvida no tempo, mais especificamente, a anisotropia de fluorescência. A partir do decaimento da anisotropia de fluorescência, podemos obter informações sobre a restrição do movimento da sonda na membrana. A análise dos coeficientes de difusão e dos tempos de correlação rotacional nos per- 
mitem fazer suposições a respeito da localização da sonda na membrana.

Os laboratórios de Biofísica do Instituto de Física da USP-SP e da FFCL da USP-RP utilizam ambas técnicas de fluorescência, e desenvolvem trabalhos em que observam a estrutura da bicamada na presença de outra molécula [2, 3], investigam a localização de proteínas na bicamada lipídica [4] e a estrutura da bicamada em diferentes fases termodinâmicas [5]. Mais recentemente, iniciaram-se trabalhos com fluorescência resolvida no tempo [6]. Ambos laboratórios têm interesse em desenvolver instrumentos de análise teórica para auxiliar na interpretação de dados experimentais de fluorescência resolvida no tempo.

A interpretação de dados experimentais de fluorescência necessita de modelos para uma ou mais localizações possíveis da sonda fluorescente na membrana, bem como para a descrição da dinâmica da sonda nesses sistemas. Esses instrumentos de análises teóricas podem ser desenvolvidos analiticamente, através da solução das equações de difusão correspondentes [7] ou através da simulação numérica para a difusão estocástica, rotacional ou translacional $[8,9,10]$.

Em relação à simulação da difusão, estudos recentes usam simulação de Monte Carlo para descrever a difusão translacional na superfície da esfera [8] e a difusão rotacional da sonda na superfície da esfera [9]. Em outro trabalho é apresentada uma outra proposta usando a técnica de simulação de dinâmica browniana [10], baseada na equação de Langevin, para a difusão rotacional restrita em um cone. Estes três trabalhos inspiraram o estudo apresentado nesta dissertação.

Neste trabalho, temos como objetivo o estudo de modelos para auxiliar na interpretação de dados experimentais de anisotropia de fluorescência da sonda fluorescente DPH (diphenylhexatriene) em bicamadas lipídicas, pelo fato dessa ser uma das moléculas mais usadas no monitoramento de bicamadas lipídicas.

No capítulo 2, é feita uma breve descrição das propriedades e características da sonda fluorescente DPH, e apresentada uma descrição física e matemática da anisotropia de fluorescência. No capítulo 3, são descritos os modelos sob investigação, sugeridos em trabalhos teóricos e experimentais. No capítulo 4, são resolvidas as equações de difusão para cada um dos modelos descritos no capítulo anterior e determinadas as expressões analíticas para a anisotropia de fluorescência. No capítulo 5, são descritas as simulações e comparados os 
resultados obtidos através das duas técnicas consideradas. No capítulo 6, são apresentadas breves conclusões. 
4 INTRODUÇÃO 


\section{Capítulo 2}

\section{Anisotropia de fluorescência}

Ao iluminarmos com luz linearmente polarizada uma amostra contendo moléculas fluorescentes, parte das moléculas dessa amostra irá absorver essa radiação, indo para um estado excitado. Na abordagem da mecânica quântica, o momento de transição é introduzido para caracterizar a transição entre um estado inicial e um estado final. O momento de transição representa o dipolo transiente resultante do deslocamento de cargas durante a transição, portanto ele não é estritamente um momento de dipolo [11].

A figura 2.1 ilustra propriedades moleculares da fluorescência, através do exemplo da molécula de antraceno, que tem dois momentos de transição de absorção, um orientado ao longo do eixo menor e o outro orientado ao longo do eixo maior, que são excitados em comprimentos de onda diferentes.
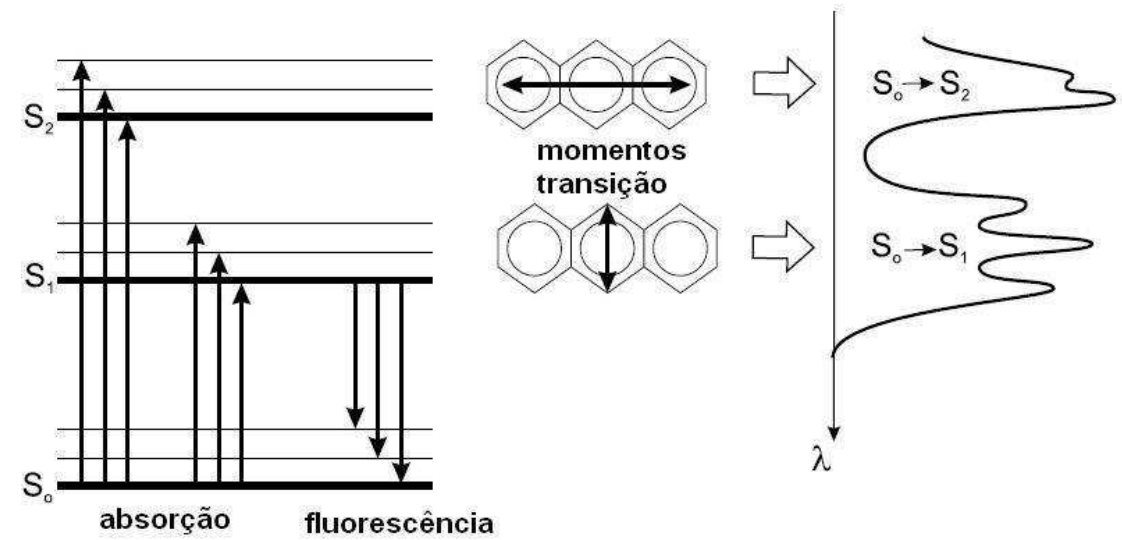

Figura 2.1: Molécula de antraceno com as duas direçôes do momento de transição de absorção, que dependem do comprimento de onda de excitação

Os momentos de dipolo de transição de absorção e de emissão podem estar alinhados 
paralelamente, ou ao longo de direções distintas. A existência de um ângulo finito entre estes dois dipolos depende dos estados eletrônicos envolvidos na transição, que são relacionados com a estrutura do fluoróforo.

Uma amostra que contém moléculas fluorescentes, predominantemente no seu estado fundamental, tem seus momentos de absorção orientados aleatoriamente. A absorção das moléculas fluorescentes é proporcional ao quadrado do cosseno do ângulo entre a direção do momento de transição de absorção e o vetor campo elétrico da luz linearmente polarizada incidente.

No retorno para o estado fundamental, há vários mecanismos para a despolarização da luz emitida pela molécula: vibração torsional, transferência de energia de excitação para outra molécula com diferente orientação e o movimento browniano rotacional durante o tempo de vida do estado excitado.

O mecanismo de interesse em nosso trabalho é a despolarização devido ao movimento browniano rotacional durante o tempo de vida do estado excitado, de moléculas com momentos de transição de absorção e de emissão paralelos.

Vamos considerar uma população de $N$ moléculas aleatoriamente orientadas segundo alguma distribuição, com momentos de transição de absorção e emissão paralelos e excitadas, no instante $t=0$, por um pulso de luz linearmente polarizada ao longo do eixo z. Como sabemos, do eletromagnetismo, a intensidade da luz é proporcional ao quadrado do campo elétrico, então a intensidade de luz absorvida pelas moléculas no instante $t=0$ é

$$
I_{0}=C E_{0}^{2}\left\langle\cos ^{2} \theta(0)\right\rangle
$$

onde $\theta(0)$ é o ângulo entre o momento de dipolo e a direção de polarização, $C$ é uma constante e $\rangle\langle\ldots\rangle\rangle$ constitui a média sobre a distribuição de orientações das moléculas excitadas. Durante o estado excitado, as moléculas rotacionam, voltando ao estado fundamental em um instante $t>0$, com o ângulo $\theta$ entre a direção de polarização e o momento de dipolo diferentes do inicial. A figura 2.2 ilustra essas mudanças do ângulo $\theta$ em função do tempo para uma molécula.

No instante de tempo $t>0$, o momento de transição das moléculas apresenta uma 


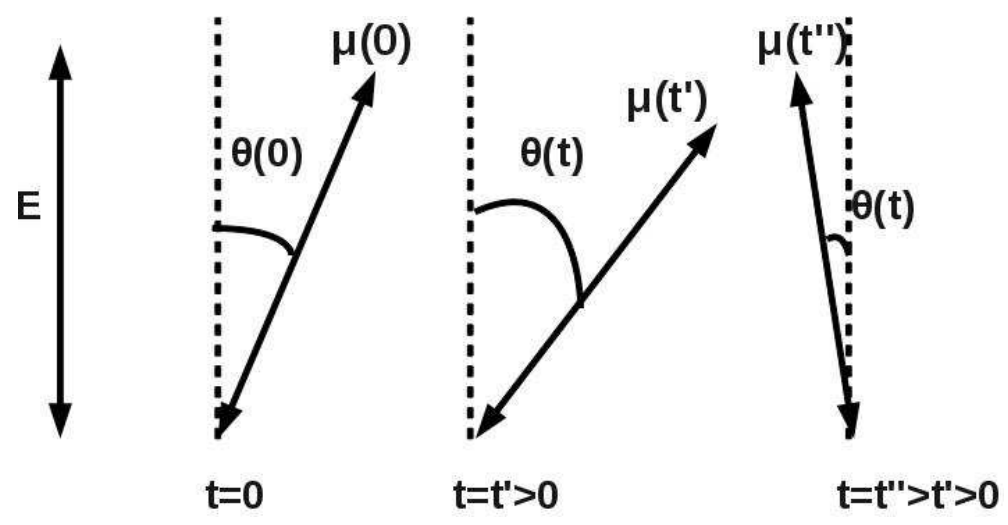

Figura 2.2: Uma molécula absorve luz linearmente polarizada no instante $t=0$ e emite a luz despolarizada, em um instante de tempo t, após o movimento rotacional aleatório dos momentos de transição colineares.

certa distribuição angular dos ângulos $\theta$, formados entre o momento de transição e o eixo z. A intensidade total de fluorescência no instante $t>0$ é obtida pela soma sobre todas as moléculas que emitem naquele instante.

Cada uma das $N$ moléculas oferece uma contribuição para as componentes da intensidade da luz emitida ao longo dos eixos $x, y, z$ que são proporcionais ao quadrado da componente do momento de transição ao longo desses eixos. A soma sobre todas as moléculas conduz às seguintes expressões para as componentes da intensidade de emissão de fluorescência:

$$
I_{x}(t)=I_{0}\left\langle\left(\mu_{x}(t)\right)^{2}\right\rangle, \quad I_{y}(t)=I_{0}\left\langle\left(\mu_{y}(t)\right)^{2}\right\rangle \quad \text { e } \quad I_{z}(t)=I_{0}\left\langle\left(\mu_{z}(t)\right)^{2}\right\rangle
$$

Por causa da simetria axial em torno do eixo $z$, a intensidade da emissão perpendicular à direção da polarização é a mesma nas direções $x$ e $y$, isto é, $I_{x}(t)=I_{y}(t)=I_{\perp}(t)$. Com isso, temos que

$$
I_{\|}=I_{0}\left\langle\cos ^{2} \theta(t)\right\rangle
$$

e como

$$
I_{\|}+2 I_{\perp}=I_{0}
$$

temos

$$
2 I_{\perp}=I_{0}\left\langle\sin ^{2} \theta(t)\right\rangle
$$


A anisotropia da fluorescência é definida como

$$
r(t)=\frac{I_{/ /}-I_{\perp}}{I_{/ /}+2 I_{\perp}}
$$

Substituindo as equações (2.3) e (2.5) na (2.6) temos que

$$
r(t)=\frac{3}{2}<\cos ^{2}(\theta(t))>-\frac{1}{2}
$$

onde $\theta(t)$ é o ângulo entre o dipolo de emissão e a direção da luz polarizada.

No caso do momento de transição de absorção e emissão paralelos, com rotação livre no solvente, inicialmente temos uma distribuição uniforme em todas as direções. A probabilidade de encontrar o dipolo excitado com direção $\left(\theta_{0}, \phi_{0}\right)$ em $t=0$ é

$$
\gamma\left(\theta_{0}\right) \propto \cos ^{2} \theta_{0}
$$

com normalização dada por

$$
\begin{array}{r}
C \int_{0}^{\pi} \cos ^{2} \theta \sin \theta d \theta \int_{0}^{2 \pi} d \phi=1 \\
C \cdot 2 \pi \frac{2}{3}=1 \rightarrow C=\frac{3}{4 \pi} .
\end{array}
$$

Portanto a média calculada na equação (2.7) em $t=0$

$$
\begin{aligned}
\left\langle\cos ^{2} \theta(0)\right\rangle & =\int d \theta_{0} \sin \theta_{0} \int d \phi_{0} \cos ^{2} \theta(0) \gamma\left(\theta_{0}\right) \\
& =\frac{3}{4 \pi} \int_{0}^{2 \pi} d \phi \int_{0}^{\pi} \cos ^{4} \theta \sin \theta d \theta=\frac{3}{5}
\end{aligned}
$$

Substituindo o resultado na equação (2.7), a emissão de anisotropia de fluorescência em $t=0$, fica

$$
r_{0}=0,4
$$

conhecida como anisotropia fundamental.

A seguir são apresentados alguns exemplos de gráficos de decaimento de fluorescência e 
decaimento de anisotropia resolvida no tempo.

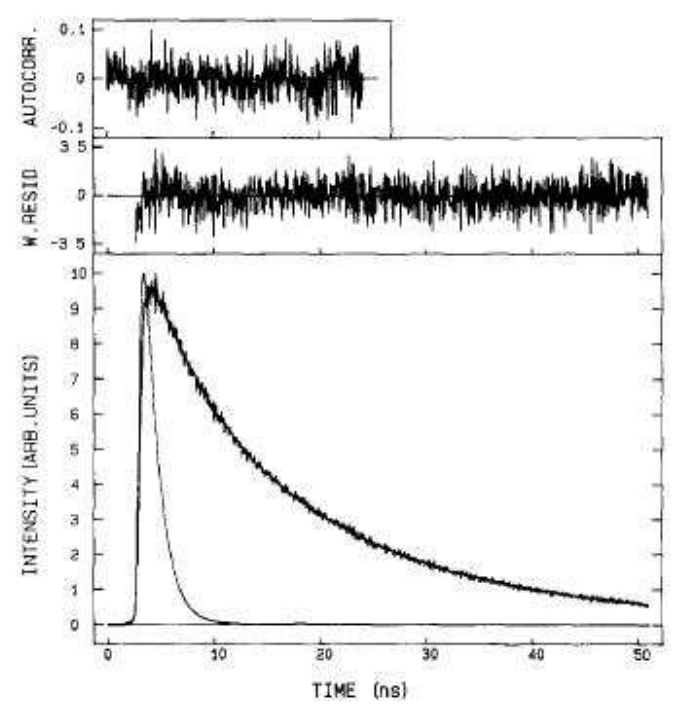

Figura 2.3: Decaimento de Fluorescência de 2-parinaroyl-PI incorporado em acetilcolina [12]
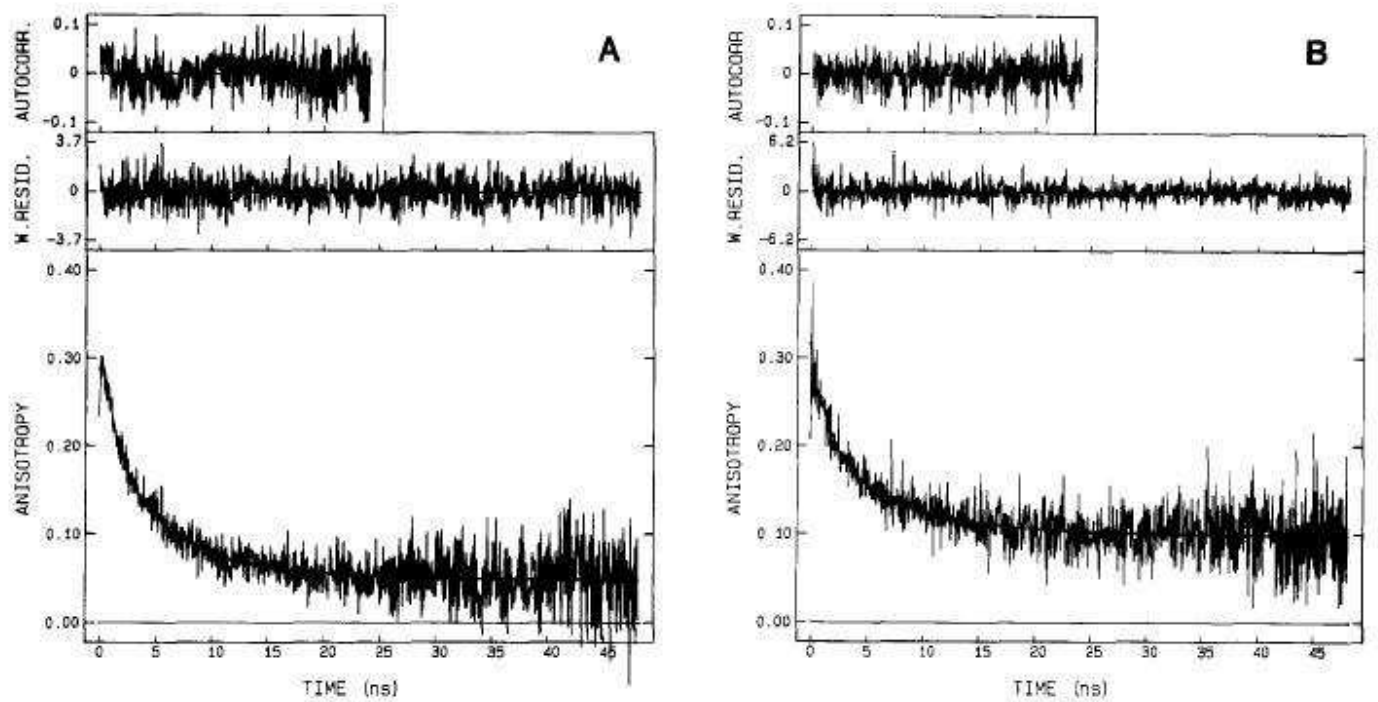

Figura 2.4: Decaimento da anisotropia de fluorescência do 2-parinaroyl-PI em vesículas lipídicas artificiais e membrana de Torpedo $m$. Medidas feitas a $4^{\circ} C$. A e B representa 2-parinaroyl-PI presente em vesícula de fosfolipídeos (PC:PI, 95:5 mol \%) e incorporado em acetilcolina, receptor rico em membrana de Torpedo $m$. [12]

As figuras 2.3 e 2.4 ilustram o decaimento e a anisotropia de fluorescência em diferentes meios. Verifica-se que o tempo de decaimento da fluorescência $(\tau \approx 50 n s)$ é bem maior que o tempo de difusão $(\tau \approx 10 n s)$.

Além disso, observa-se na figura 2.4, que o valor da anisotropia para tempos longos não é zero, como para uma molécula rotacionando livremente em um solvente. O movimento da 
molécula está restrito pelas moléculas constituintes da vesícula, que pode ser verificado pelos valores de anisotropia, para tempos longos, nos dois gráficos.

No caso em que os momentos de transição e emissão não são paralelos, existirá um ângulo entre a direção do momento de absorção e o de emissão. A figura 2.5 ilustra a disposição dos momentos de transição de absorção e de emissão $\vec{\mu}_{E}$ e $\vec{\mu}_{E}$, que fazem ângulos $\theta_{A}$ e $\theta_{E}$ com a direção de polarização. Podemos perceber que é formado um triângulo esférico, os lados

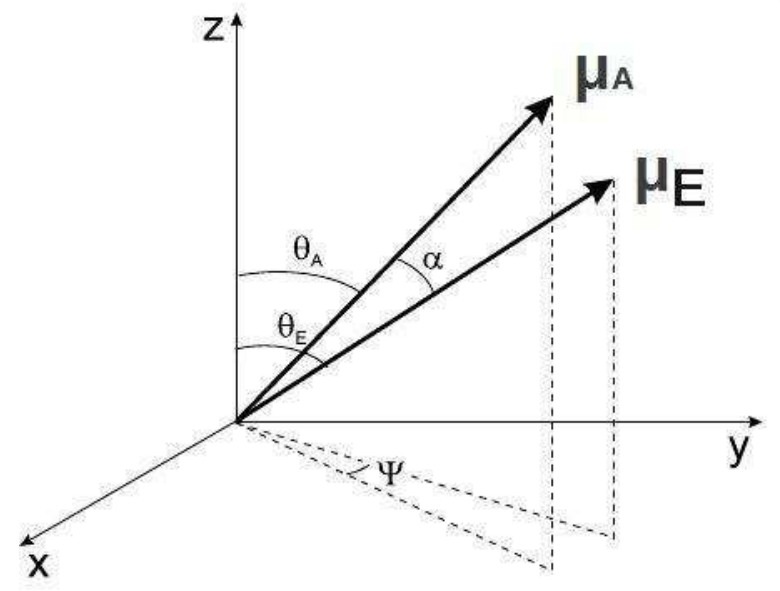

Figura 2.5: Momento de transição de absorção e de emissão não paralelos

do triângulo são os arcos esféricos formados pelos momento de absorção e de emissão, com relação à direção de polarização e o arco esférico entre os dois momentos de transição.

De acordo com a trigonometria esférica, apresentada no apêndice (E), $\cos \theta_{E}$ pode ser escrito em termos dos ângulos do dipolo de absorção $\theta_{A}$ e do ângulo $\alpha$ entre os dois dipolos como

$$
\cos \theta_{E}=\cos \theta_{A} \cos \alpha+\cos \psi \sin \theta_{A} \sin \alpha,
$$

onde $\psi$ é o ângulo entre os planos $\left(O z, \vec{\mu}_{A}\right)$ e $\left(O z, \vec{\mu}_{E}\right)$. Elevando ao quadrado a equação (2.12), temos

$$
\cos ^{2} \theta_{E}=\cos ^{2} \theta_{A} \cos ^{2} \alpha+2 \cos \theta_{A} \cos \alpha \cos \psi \sin \theta_{A} \sin \alpha+\cos ^{2} \psi \sin ^{2} \theta_{A} \sin ^{2} \alpha .
$$

Levando em conta que $\alpha$ é constante e que todos os valores de $\psi$ são equiprováveis, portanto

$$
\langle\cos \psi\rangle=0 \quad e \quad\left\langle\cos ^{2} \psi\right\rangle=\frac{1}{2},
$$


assim temos

$$
\begin{aligned}
\left\langle\cos ^{2} \theta_{E}\right\rangle & =\cos ^{2} \alpha\left\langle\cos ^{2} \theta_{A}\right\rangle+\frac{1}{2} \sin ^{2} \alpha\left\langle\sin ^{2} \theta_{A}\right\rangle \\
& =\cos ^{2} \alpha\left\langle\cos \theta_{A}\right\rangle+\frac{1}{2}\left(1-\cos ^{2} \alpha\right)\left(1-\left\langle\cos ^{2} \theta_{A}\right\rangle\right) \\
& =\frac{3}{2} \cos ^{2} \alpha\left\langle\cos ^{2} \theta_{A}\right\rangle-\frac{1}{2}\left\langle\cos ^{2} \theta_{A}\right\rangle-\frac{1}{2} \cos ^{2} \alpha+\frac{1}{2}
\end{aligned}
$$

Substituindo a equação (2.15) na equação (2.7) para a anisotropia de fluorescência, temos

$$
r(t)=\left(\frac{3}{2}\left\langle\cos ^{2} \theta_{A}\right\rangle-\frac{1}{2}\right)\left(\frac{3}{2} \cos ^{2} \alpha-\frac{1}{2}\right) .
$$

A diferença entre as expressões para a anisotropia de fluorescência para o caso colinear e o caso não colinear é apenas um fator multiplicativo, que depende de $\alpha$.

Usamos o mesmo raciocínio desenvolvido para o cálculo da anisotropia fundamental, para momentos de transição paralelos, para calcular a média $\left\langle\cos ^{2} \theta_{A}\right\rangle$, sendo assim

$$
\frac{3}{2}\left\langle\cos ^{2} \theta_{A}\right\rangle-\frac{1}{2}=0,4
$$

Portanto, para momentos de transição não paralelos, a anisotropia fundamental irá depender do ângulo entre os momentos de absorção e emissão, ou seja,

$$
r(0)=0,4\left(\frac{3}{2} \cos ^{2} \alpha-\frac{1}{2}\right)
$$

Isso mostra que a anisotropia fundamental pode variar entre $-0,2 \leq r(0) \leq 0,4$. O limite superior corresponde ao caso colinear $(\alpha=0)$, deduzido anteriormente, equação (2.11).

A anisotropia em $t=0, r(0)$, chamada de anisotropia de emissão, fornece informação importante sobre o fluoróforo. A figura 2.6 ilustra este fato.

A figura 2.6, mostra o gráfico anisotropia fundamental, $r(0)$, em função do comprimento de onda de excitação, para a molécula de perilene. Através do gráfico, podemos observar que existe um ângulo entre o momento de transição de absorção e de emissão, e que esse ângulo pode aumentar ou diminuir, de acordo com os comprimentos de onda de excitação. 


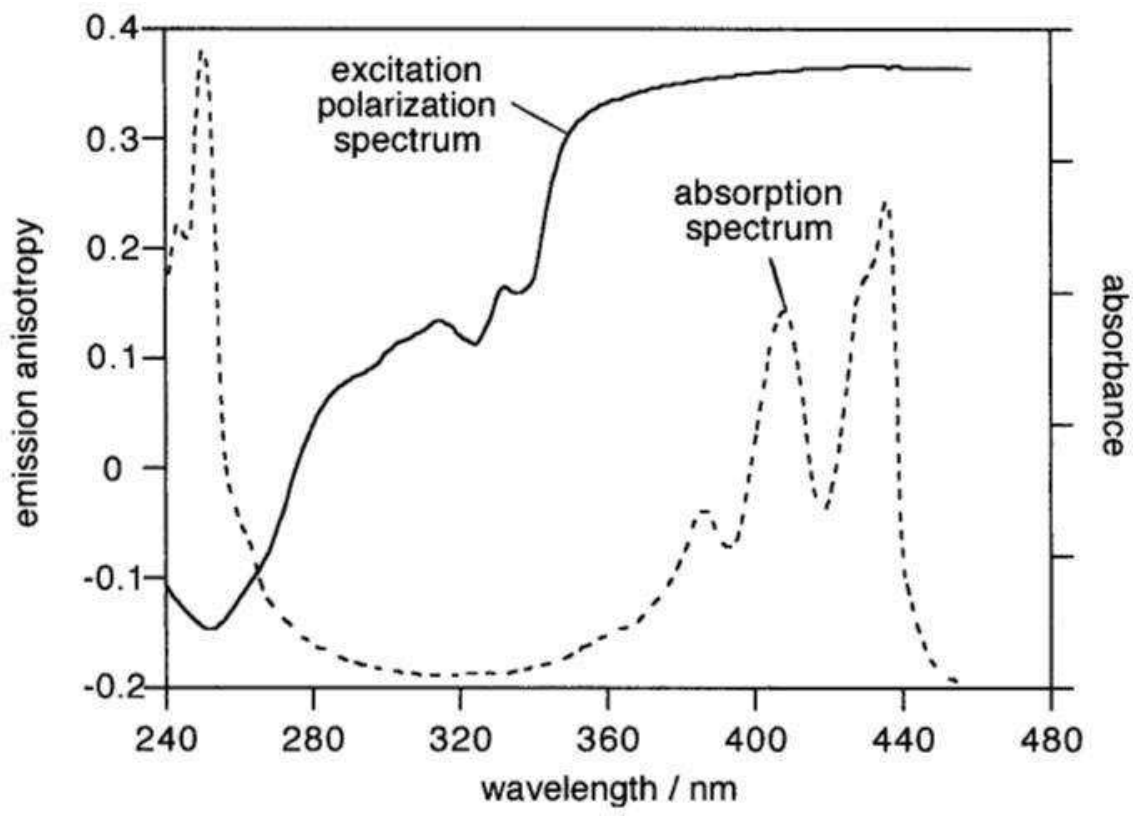

Figura 2.6: Perilene em propane 1,2-diol a $60^{\circ} \mathrm{C}$ 


\section{Capítulo 3}

\section{Modelos para difusão rotacional}

Nossos modelos tentam descrever o comportamento da anisotropia de fluorescência da molécula DPH (1,6 Diphenyl - 1,3,5 Hexatriene). A sonda fluorescente DPH, representada na figura 3.1, é uma molécula de simetria uniaxial, que tem como volume de revolução um elipsóide prolato, com dois eixos iguais e um eixo diferente, maior que os outros dois. Os momentos de transição de absorção (A) e emissão (E) estão alinhados ao longo do eixo maior da molécula [13, 1].

As propriedades rotacionais dos elipsóides são descritas em termos de dois coeficientes de difusão rotacional, $D_{\|}$e $D_{\perp}$, ao redor de cada um dos eixos. Então uma molécula como o DPH poderia exibir dois tempos de correlação de rotação. A figura 3.1 mostra a estrutura da molécula de DPH, o elipsóide correspondente ao volume ocupado por ela sob rotação, bem como a localização dos momentos de transição de absorção (A) e emissão (E).

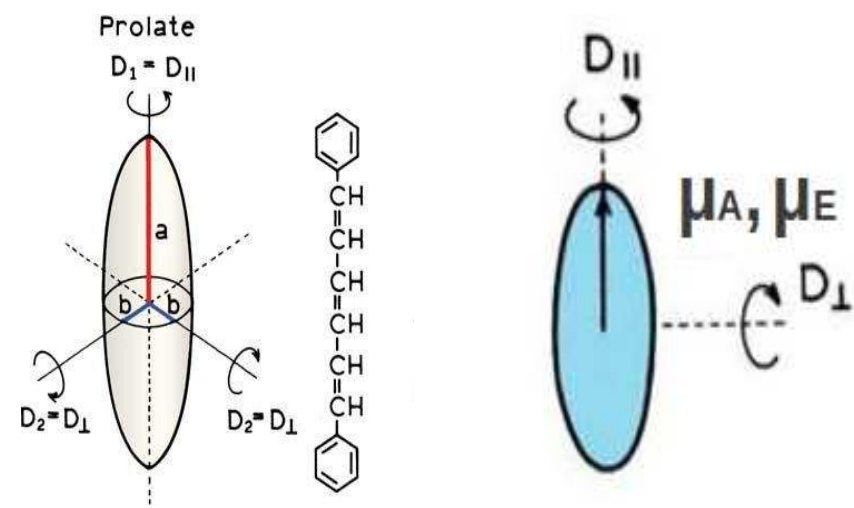

Figura 3.1: Esquerda - Volume ocupado pela molécula de DPH, com seus eixos de rotaçôes e a sua estrutura molecular. Direita - Volume ocupado levando em consideração as direçôes dos momentos de absorção e emissão. 
Na rotação livre na solução homogênea o DPH pode ter seu comportamento considerado como um rotor isotrópico [1, 14]. A sua rotação ao redor do eixo maior $\left(D_{\|}\right)$não desloca os momentos de transição colineares e assim não decresce a anisotropia, ou seja, não despolariza a luz emitida. Somente a rotação que desloca os momentos de transição resulta em despolarização $[13,1,14]$. Com isso temos que apenas um dos tempos de correlação rotacional e um dos coeficientes de difusão, $D_{\perp}$, contribuem para a anisotropia de fluorescência.

Dados experimentais de intensidade e despolarização de fluorescência do DPH em meio isotrópico como glicerina e parafina líquida, levando em conta várias temperaturas, são apresentados em [13]. O trabalho apresenta dados que dão suporte à ideia da existência de um único tempo de correlação. Em outro trabalho [14], usando a técnica experimental de domínio da frequência, que difere da apresentada em [13], e usando um outro solvente, é mostrada a existência de um único tempo de correlação rotacional para o DPH rotacionando livremente.

Kinosita et al. [7] foi um dos pioneiros no desenvolvimento do modelo teórico para moléculas fluorescentes incorporadas entre as cadeias hidrofóbicas da bicamada lipídica. Segundo o autor, o fluoróforo tem seu movimento de rotação restrito, dentro de um cone. A partir desse modelo determina-se um parâmetro de ordem que descreve a rigidez das moléculas da membrana. Em outro trabalho [13], são apresentados resultados experimentais para DPH em membrana de DPPC, em que os parâmetros são extraídos levando em conta o modelo do cone. O trabalho é baseado na hipótese de que o DPH fica somente entre as cadeias dos lipídios constituintes da membrana.

Mais recentemente, foi introduzida a ideia de que haveria duas populações na distribuição do DPH em membranas. Levine et al. [15], em trabalho de 1996, interpreta os dados da intensidade de fluorescência em termos de uma dupla exponencial, com dois tempos de decaimento de fluorescência, que corresponderiam a dois meios distintos. O ajuste dos dados, que não são apresentados, levam os autores à proposta de uma fração grande da população do fluoróforo no plano paralelo à superfície e no centro da bicamada. Em trabalho anterior, de 1994, E. Pebay-Peyroula et al. [16], utilizando a técnica de difração de nêutrons, e analisando o respectivo fator de espalhamento, também propõem a existência de duas populações, mas com localização diferente da população de fluoróforo com eixo paralelo ao plano: 
os fluoróforos estariam próximos à superfície da membrana. No trabalho mais recente de Konopasek et al. [17], a análise do deslocamento do espectro de intensidade de fluorescência estática os leva a ter a mesma conclusão de E. Pebay et al.[16], isto é, a de duas populações de fluoróforos na bicamada. Não há trabalhos conclusivos com relação à real distribuição dessas populações.

Como contribuição para o esclarecimento dessa questão, iremos analisar três modelos para fluoróforos uniaxiais, como o DPH: i) livre em uma solução homogênea; ii) com sua localização entre as cadeias lipídicas da bicamada lamelar, tendo seu movimento restrito dentro de um cone e iii) rotação paralela à superfície da membrana.

\subsection{Rotação livre}

No modelo de rotação livre, estamos considerando que os momentos de transição alinhados com relação ao eixo maior da molécula rotacionam livremente, de forma aleatória, em uma solução homogênea. A figura 3.2 descreve essa situação para uma distribuição de moléculas em uma dada solução. A equação de difusão a ser considerada é a equação para a distribuição de probabilidade $G(\theta, t)$, onde $\theta$ é o ângulo entre o momento de transição e a direção de polarização.
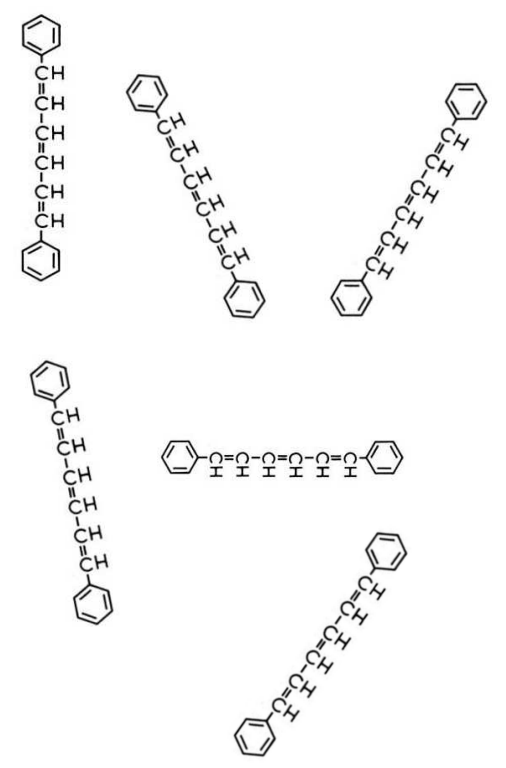

Figura 3.2: Moléculas de DPH, rotacionando livremente na solução 


\subsection{Rotação paralela à superfície da membrana}

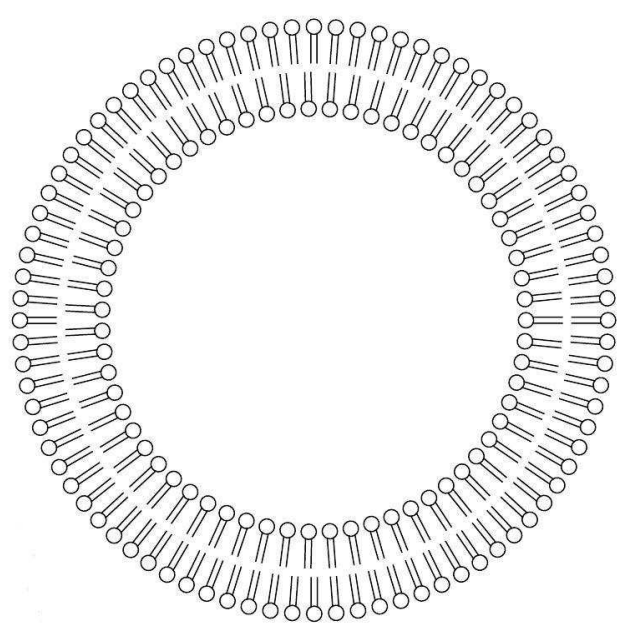

Figura 3.3: Membrana em vesículas esférica

Nesse outro modelo, a molécula de DPH rotaciona no plano paralelo à superfície da vesícula lipídica, figura 3.4(a). A bicamada lipídica é representada por uma esfera, levando em conta que estamos considerando que a membrana, onde será incorporada a sonda fluorescente define uma simetria esférica. A equação de difusão é resolvida em um referencial fixo na superfície da esfera $R$, como na figura 3.4(b). Obtemos a distribuição de probabilidade $G(\phi, t)$, onde $\phi$ é o ângulo de rotação do dipolo no plano tangente à superfície esférica neste referencial. Para as componentes da intensidade da luz emitida pela distribuição de dipolos, temos que considerar uma distribuição de planos paralelos à superfície esférica, ou seja um conjunto $(\alpha, \beta)$, como na figura 3.4(a). A anisotropia deve ser calculada no referencial $R^{\prime}$.

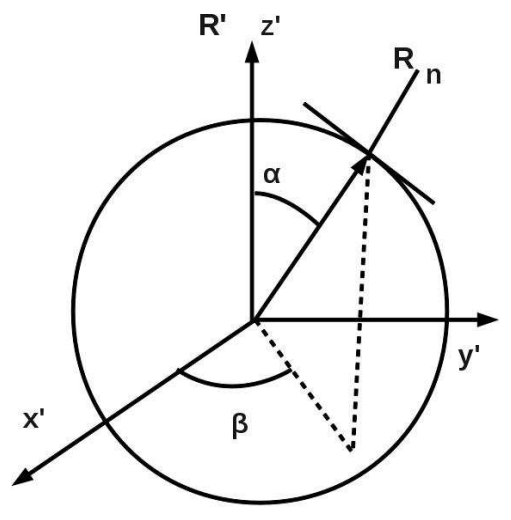

(a) Esfera onde serão distribuídos os planos paralelos à superfície

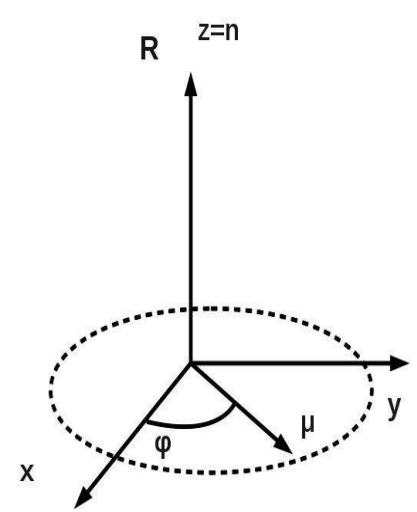

(b) Referencial onde será resolvida a equação da difusão

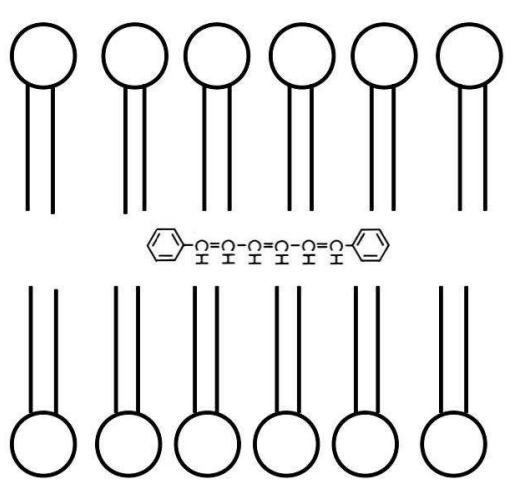

(c) Esquema de como a sonda DPH pode estar na membrana

Figura 3.4 


\subsection{Rotação restrita no cone}

Nesse modelo, o DPH está localizado entre as cadeias lipídicas das moléculas constituintes da bicamada. Devido à localização do DPH, seu movimento é restrito pelas cadeias lipídicas e por conta da simetria do DPH, seu movimento rotacional fica restrito ao que se assemelha a um cone. A equação de difusão é resolvida em um referencial fixo na superfície da esfera, como na figura 3.5(a). Obtemos a distribuição de probabilidade $G(\theta, \phi, t)$, onde $\theta$ e $\phi$ são os ângulos de rotação do dipolo no referencial tangente à superfície esférica, $R$. Para as componentes da intensidade da luz emitida pela distribuição de dipolos, temos que considerar uma distribuição de referenciais na superfície esférica, ou seja um conjunto $(\alpha, \beta)$, como na figura 3.4(a).

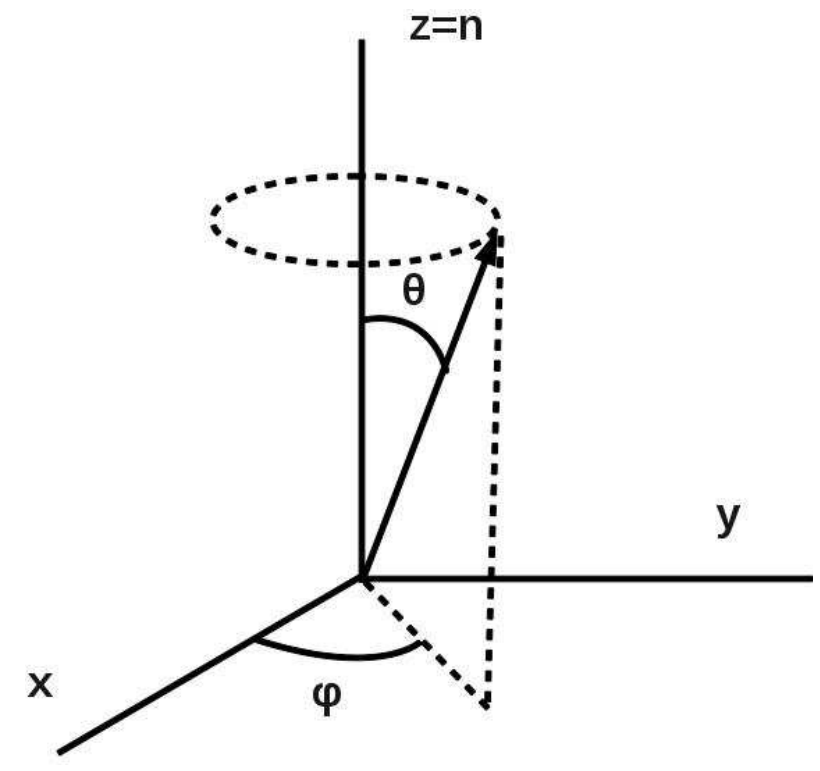

(a) Referencial onde será resolvida a equação da difusão, para o movimento restrito no cone
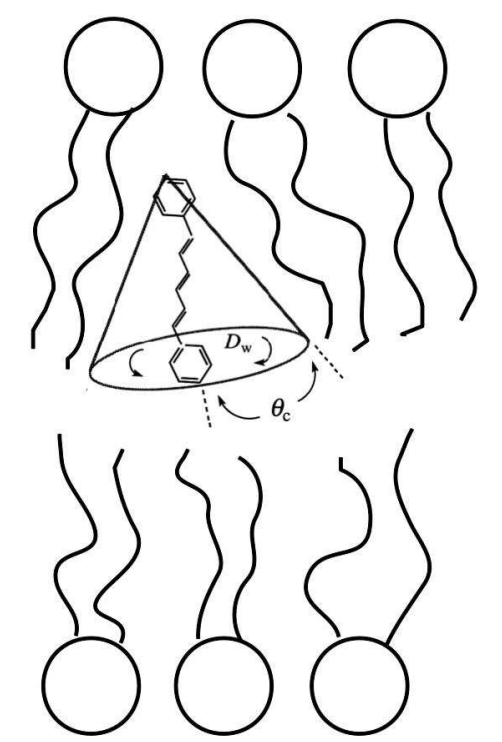

(b) Esquema de como a sonda $\mathrm{DPH}$ pode estar incorporada na membrana

Figura 3.5 


\section{Capítulo 4}

\section{Difusão rotacional e anisotropia de}

\section{fluorescência - cálculo analítico}

A anisotropia de fluorescência média é uma grandeza que varia no tempo de acordo com a configuração orientacional instantânea dos momentos de emissão. Essa configuração é descrita pela solução da equação de difusão rotacional, cuja solução depende das condições de contorno impostas. Neste capítulo, vamos resolver a equação de difusão rotacional e obter expressões para a anisotropia resolvida no tempo, $r(t)$, para os três modelos propostos no capítulo anterior.

\subsection{A equação da difusão rotacional - 3D}

Nos nossos modelos estamos considerando que a rotação do momento de dipolo de simetria uniaxial. Então definimos que $G$ é a probabilidade de encontrar essa sonda em uma dada configuração espacial $\Omega(\theta, \phi, t)$ no instante $t$. Na figura 4.1 os ângulos $\theta$ e $\phi$ correspondem aos ângulos polar e azimutal, respectivamente.

A difusão rotacional desse momento de dipolo $\vec{\mu}$ é obtida através da solução da equação de difusão

$$
\frac{\partial}{\partial t} G(\theta, \phi, t)=D_{r} \nabla^{2} G(\theta, \phi, t)
$$

em que $D_{r}$ é o coeficiente de difusão rotacional e $G(\theta, \phi, t)$ é a probabilidade descrita ante- 


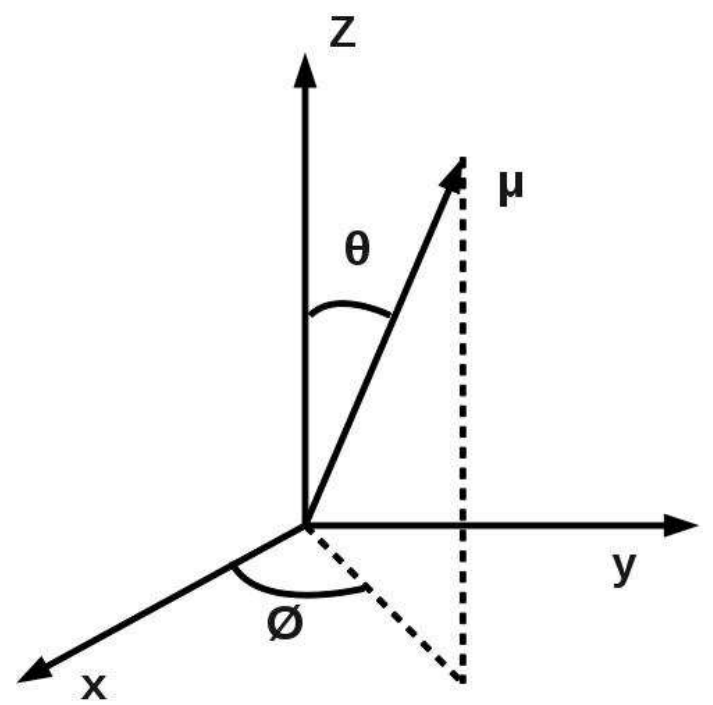

Figura 4.1: Dipolo associado a sonda fluorescente com suas respectivas coordenadas espacial

riormente.

Devido à simetria do problema, escrevemos o laplaciano na seguinte forma:

$$
\nabla^{2}=\frac{1}{r^{2}} \frac{\partial}{\partial r}\left(r^{2} \frac{\partial}{\partial r}\right)+\frac{1}{r^{2} \sin \theta} \frac{\partial}{\partial \theta}\left(\sin \theta \frac{\partial}{\partial \theta}\right)+\frac{1}{r^{2} \sin ^{2} \theta} \frac{\partial^{2}}{\partial \phi^{2}}
$$

onde $\theta$ e $\phi$ são os ângulos polar e azimutal mostrados na figura 4.1. A coordenada radial é uma constante, o que permite escrever o laplaciano apenas em termos das dependências angulares:

$$
\nabla^{2}=\frac{1}{\sin \theta} \frac{\partial}{\partial \theta}\left(\sin \theta \frac{\partial}{\partial \theta}\right)+\frac{1}{\sin ^{2} \theta} \frac{\partial^{2}}{\partial \phi^{2}}
$$

para $R=1$. Substituindo a equação (4.3) na equação (4.1), obtemos a seguinte expressão para a equação da difusão

$$
\frac{\partial}{\partial t} G(\theta, \phi, t)=\frac{D_{r}}{\sin \theta} \frac{\partial}{\partial \theta}\left(\sin \theta \frac{\partial}{\partial \theta} G(\theta, \phi, t)\right)+\frac{D_{r}}{\sin ^{2} \theta} \frac{\partial^{2}}{\partial \phi^{2}} G(\theta, \phi, t)
$$

que é uma equação diferencial parcial com 3 variáveis, cuja solução é obtida através do método de separação de variáveis.

Esta técnica separa a equação diferencial parcial de $n$ variáveis em $n$ equações diferenciais ordinárias. A cada separação é introduzida uma constante arbitrária de separação. Então para $n$ variáveis teremos $n-1$ constantes, determinadas de acordo com as condições impostas pelo problema a ser resolvido [18]. 
Para aplicarmos o método de separação de variáveis ao problema, começamos escrevendo a probabilidade $G(\theta, \phi, t)$ como sendo um produto de três funções, uma em cada variável

$$
G(\theta, \phi, t)=H(\theta) F(\phi) T(t)
$$

Por simplicidade, utilizaremos $H(\theta)=H, F(\phi)=F$ e $T(t)=T$. Substituindo a equação (4.5) na equação (4.4), temos

$$
\frac{\partial}{\partial t}(H F T)=\frac{D_{r}}{\sin \theta} \frac{\partial}{\partial \theta}\left(\sin \theta \frac{\partial}{\partial \theta}(H F T)\right)+\frac{D_{r}}{\sin ^{2} \theta} \frac{\partial^{2}}{\partial \phi^{2}}(H F T)
$$

Se levarmos em conta a dependência de cada uma das três funções, a equação (4.6) passa a ser escrita na forma

$$
H F \frac{d T}{d t}=\frac{F T D_{r}}{\sin \theta} \frac{d}{d \theta}\left(\sin \theta \frac{d H}{d \theta}\right)+\frac{H T D_{r}}{\sin ^{2} \theta} \frac{d^{2} F}{d \phi^{2}}
$$

Dividindo ambos os lados da equação (4.7) por HFT, obtemos

$$
\frac{1}{T} \frac{d T}{d t}=\frac{D_{r}}{H \sin \theta} \frac{d}{d \theta}\left(\sin \theta \frac{d H}{d \theta}\right)+\frac{D_{r}}{F \sin ^{2} \theta} \frac{d^{2} F}{d \phi^{2}} .
$$

Sendo $\theta, \phi$ e $t$ variáveis independentes, podemos escrever

$$
\begin{gathered}
\frac{1}{D_{r}} \frac{1}{T} \frac{d T}{d t}=\frac{1}{H \sin \theta} \frac{d}{d \theta}\left(\sin \theta \frac{d H}{d \theta}\right)+\frac{1}{F \sin ^{2} \theta} \frac{d^{2} F}{d \phi^{2}}=-k \\
\frac{1}{F} \frac{d^{2} F}{d \phi^{2}}=m^{2}
\end{gathered}
$$

e

$$
\frac{\sin \theta}{H} \frac{d}{d \theta}\left(\sin \theta \frac{d H}{d \theta}\right)-m^{2}=-k \sin \theta
$$

onde $k$ é uma constante positiva e $m$ um inteiro. Assim, a equação diferencial ordinária com relação à variável $t$ é

$$
\frac{d T(t)}{d t}=-k D_{r} T(t)
$$


uma equação diferencial homogênea de primeira ordem, cuja solução é

$$
T(t)=T(0) \exp \left(-k D_{r} t\right)
$$

A equação diferencial correspondente à variável $\phi$ é

$$
\frac{d^{2} F(\phi)}{d \phi^{2}}=-m^{2} F(\phi)
$$

e sua solução é

$$
F(\phi)=\sum_{m=0}^{\infty}\left(A_{m} \cos m \phi+B_{m} \sin m \phi\right) .
$$

A equação diferencial com relação à variável $\theta$ é

$$
\sin \theta \frac{d}{d H}\left(\sin \theta \frac{d H(\theta)}{d \theta}\right)+\left(k \sin ^{2} \theta-m^{2}\right) H(\theta)=0
$$

chamada de equação associada de Legendre e $k=l(l+1)$ por convenção. Então temos $m$ e $l$ constantes, sendo $m$ um inteiro e $l$ um número real obtido, de acordo com as condições de contorno do problema em $\theta$. Podemos reescrever a equação (4.16) com um outro formato. Fazendo a mudança de variável $\mu=\cos \theta$, temos

$$
\frac{d}{d \mu} \frac{d \mu}{d \theta} \Longrightarrow-\sin \theta \frac{d}{d \mu}
$$

Usando a relação trigonométrica

$$
\cos ^{2} \theta+\sin ^{2} \theta=1 \Longrightarrow \sin ^{2} \theta=1-\mu^{2}
$$

e substituindo as equações (4.17) e (4.18) na equação (4.16) obtemos

$$
\left(1-\mu^{2}\right) \frac{d^{2} H(\theta)}{d \mu^{2}}-2 \mu \frac{d H(\theta)}{d \mu}+\left[l(l+1)-\frac{m^{2}}{1-\mu^{2}}\right] H(\theta)=0 .
$$

Em resumo temos as soluções das equações diferenciais para $t$ e $\phi$ respectivamente

$$
T(t)=T(0) \exp \left[-l(l+1) D_{r} t\right]
$$




$$
F(\phi)=\sum_{m=0}^{\infty}\left(A_{m} \cos m \phi+B_{m} \sin m \phi\right),
$$

enquanto a solução da equação diferencial (4.19) com respeito a $\theta$ vai depender da geometria dos modelos.

\subsection{A equação da difusão rotacional - 2D}

Nesse outro modelo consideramos também uma sonda de simetria uniaxial, porém com seu movimento rotacional restrito a um plano. Então definimos da mesma forma que no modelo 3D uma função $G$, que é a probabilidade de se encontrar esse dipolo em uma dada configuração do plano $\Omega(\phi, t)$ no tempo $t$. A figura 4.2 representa o plano onde rotaciona o dipolo e o ângulo polar $\phi$.

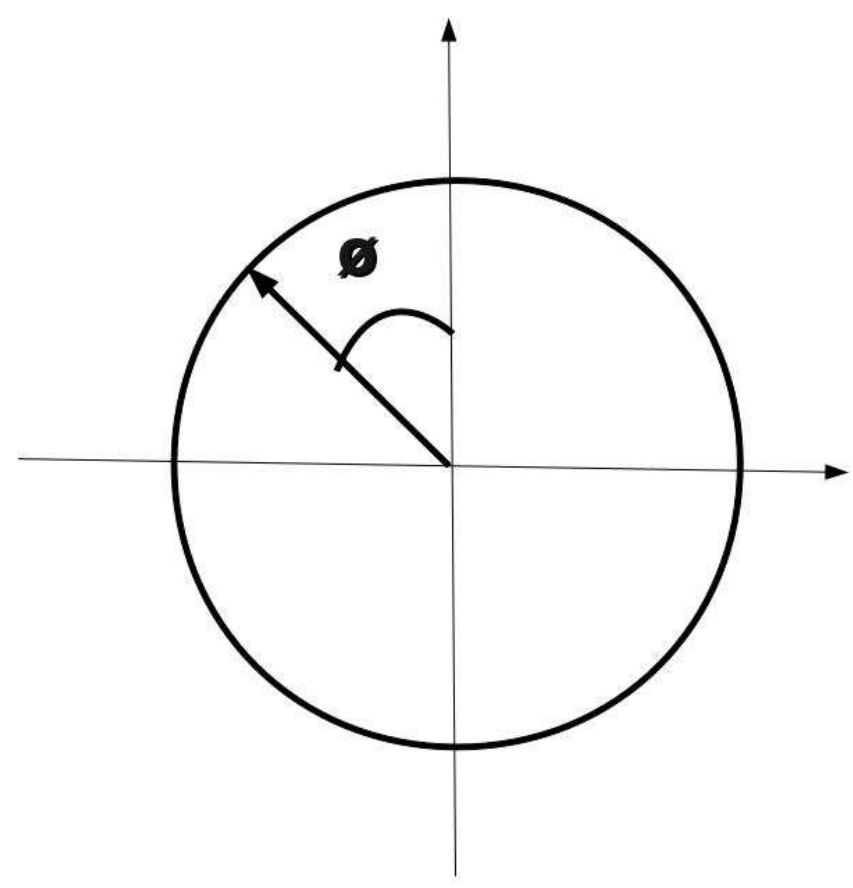

Figura 4.2: Dipolo associado a sonda fluorescente com sua respectiva coordenada polar

A função $G$ é obtida através da solução da equação da difusão bidimensional

$$
\frac{\partial}{\partial t} G(\phi, t)=D_{r} \nabla^{2} G(\phi, t)
$$

em que $D_{r}$ é o coeficiente de difusão e $G(\phi, t)$ é a probabilidade.

O laplaciano da equação (4.22) é dado em coordenadas polares devido à simetria do 
problema

$$
\nabla^{2}=\frac{1}{r} \frac{\partial}{\partial r}\left(r \frac{\partial}{\partial r}\right)+\frac{1}{r^{2}} \frac{\partial^{2}}{\partial \phi^{2}}
$$

onde $\phi$ é o ângulo polar.

O problema não tem dependência radial, portanto a coordenada radial é uma constante, simplificando o laplaciano apenas para dependência angular

$$
\nabla^{2}=\frac{1}{r^{2}} \frac{\partial^{2}}{\partial \phi^{2}}
$$

Substituindo a equação (4.24) na equação (4.22) e fazendo $R=1$, temos

$$
\frac{\partial}{\partial t} G(\phi, t)=D_{r} \frac{\partial^{2}}{\partial \phi^{2}} G(\phi, t)
$$

A solução da equação (4.25) é obtida através do uso do método de separação de variáveis, escrevendo a função $G(\phi, t)$ como o produto de 2 funções,

$$
G(\phi, t)=T(t) Q(\phi)
$$

que por simplicidade apenas escreveremos $T(t)=T$ e $Q(\phi)=Q$. Substituímos a equação (4.26) na equação (4.25) obtemos

$$
\frac{\partial}{\partial t} T Q=D_{r} \frac{\partial^{2}}{\partial \phi^{2}} T Q
$$

Levando em conta as dependências de cada uma das funções, podemos escrever a equação (4.27) como

$$
Q \frac{d}{d t} T=D_{r} T \frac{d^{2}}{d \phi^{2}} Q
$$

Dividimos ambos os lados da equação (4.28) por $T Q$ e igualando a uma constante temos

$$
\frac{1}{D_{r} T} \frac{d}{d t}=\frac{1}{Q} \frac{d^{2}}{d \phi^{2}} Q=-\lambda
$$


A equação (4.29) vai dar origem a 2 equações diferenciais. Uma com relação à variável $t$

$$
\frac{d T(t)}{d t}=-\lambda D_{r} T(t)
$$

cuja solução é

$$
T(t)=T(0) \exp \left(-\lambda D_{r} t\right)
$$

A equação diferencial com relação à variável $\phi$ é

$$
\frac{d^{2}}{d \phi^{2}} Q(\phi)+\lambda Q(\phi)=0
$$

e tomando $\lambda=l^{2}$, sendo $l$ inteiros, temos a seguinte solução

$$
Q(\phi)=\sum_{l=0}^{\infty}\left[A_{l} \cos l \phi+B_{l} \sin l \phi\right]
$$

Assim, a solução da equação de difusão no plano é dada por

$$
G(\phi, t)=\sum_{l=0}^{\infty}\left[A_{l} \cos l \phi+B_{l} \sin l \phi\right] T_{l}(0) \exp \left(-l^{2} D_{r} t\right)
$$

\subsection{A função densidade probabilidade $\mathrm{G}(\Omega, \mathrm{t})$}

Nesta seção vamos desenvolver o cálculo analítico da função densidade probabilidade para a orientação do dipolo emissor, no instante $t$, para cada um dos 3 modelos.

\subsubsection{Rotação livre}

Para o modelo de rotação livre, devemos ter uma solução para a função distribuição de probabilidade $F(\phi)$ que seja independente de $\phi$. A equação (4.21) só pode ser assim satisfeita para $m=0$, com $F(\phi)=A_{0}$. Com $m=0$, a equação (4.19) passa a ser uma equação diferencial de Legendre, cuja solução é dada por

$$
H(\theta)=\sum_{l} A_{l} P_{l}(\cos \theta), \quad l=0,1,2, \ldots
$$


Substituindo as equações (4.20) e (4.35) em (4.5), obtemos

$$
G(\theta, t)=\sum_{l} A_{l} T_{l}(0) P_{l}(\cos \theta) \exp \left[-l(l+1) D_{r} t\right]
$$

onde a cosntante $A_{0}$ foi incorporada nos coeficientes da função $H(\theta)$.

O objetivo agora é determinarmos o valor das constantes $A_{l}$ e $T_{l}(0)$. Para isso multiplicamos ambos os membros da equação (4.36) por $P_{k}(\cos \theta)$,

$$
G(\theta, t) P_{k}(\cos \theta)=\sum_{l} A_{l} T_{l}(t) P_{l}(\cos \theta) P_{k}(\cos \theta) \exp \left[\left(-l(l+1) D_{r} t\right]\right.
$$

Em seguida, integramos os dois lados da equação em relação a $\theta$ e para $t=0$ ficamos com

$$
\int_{-1}^{1} G(\theta, 0) P_{k}(\cos \theta) d(\cos \theta)=\sum_{l} A_{l} T_{l}(0) \int_{-1}^{1} P_{l}(\cos \theta) P_{k}(\cos \theta) d(\cos \theta)
$$

Usando a propriedade de ortogonalidade dos polinômios de Legendre, em que

$$
\int_{-1}^{1} P_{l}(\cos \theta) P_{k}(\cos \theta) d(\cos \theta)=\frac{2}{2 k+1} \delta_{l k}
$$

com

$$
\delta_{n n^{\prime}}=\left\{\begin{array}{ccc}
1, & \text { se } & l=k \\
0, & \text { se } & l \neq k
\end{array}\right.
$$

temos

$$
A_{k} T_{k}(0)=\frac{2 k+1}{2} \int_{-1}^{1} G(\theta, 0) P_{k}(\cos \theta) d(\cos \theta) .
$$

Em $t=0$, definimos $\theta=\theta_{0}$, ou

$$
G\left(\theta_{0}, 0\right)=\delta\left(\cos \theta_{0}\right)
$$


com a função delta de Dirac

$$
\delta\left(\cos \theta_{0}\right)=\left\{\begin{array}{ccc}
0, & \text { se } & \cos \theta \neq \cos \theta_{0} \\
\infty, & \text { se } & \cos \theta=\cos \theta_{0}
\end{array}\right.
$$

ficando

$$
A_{k} T_{k}(0)=\frac{2 k+1}{2} \int_{-1}^{1} \delta\left(\cos \theta_{0}\right) P_{k}(\cos \theta) d(\cos \theta)
$$

$\mathrm{Ou}$

$$
A_{k} T_{k}(0)=\frac{2 k+1}{2} P_{k}\left(\cos \theta_{0}\right)
$$

Finalmente, podemos a partir da equação (4.36), escrever a probabilidade do dipolo estar em uma configuração $\theta$, em $t$, dada uma orientação inicial $\theta_{0}$, em $t=0, G\left(\theta, t ; \theta_{0}, 0\right)$, a equação

$$
G\left(\theta, t ; \theta_{0}, 0\right)=\sum_{l} \frac{2 l+1}{2} P_{l}\left(\cos \theta_{0}\right) P_{l}(\cos \theta) \exp \left(-l(l+1) D_{r} t\right)
$$

\subsubsection{Rotação no plano}

A partir da equação (4.34) devemos calcular as constantes $A_{l} T_{l}(0)$ e $B_{l} T_{l}(0)$. Para isso começamos multiplicando ambos os membros da equação (4.34) por cos $n \phi$ e integramos em relação a $\phi$. Com isso temos

$$
\begin{array}{r}
\int_{0}^{2 \pi} \cos n \phi G(\phi, t) d \phi=\sum_{l}\left[A_{l} T_{l}(0) \int_{0}^{2 \pi} \cos n \phi \cos l \phi d \phi\right. \\
\left.+B_{l} T_{l}(0) \int_{0}^{2 \pi} \cos n \phi \sin l \phi d \phi\right] \exp \left(-l^{2} D_{r} t\right) .
\end{array}
$$

Agora multiplicamos ambos os membros da equação (4.34) por sin n申 e integramos. Com isso temos

$$
\begin{array}{r}
\int_{0}^{2 \pi} \sin n \phi G(\phi, t) d \phi=\sum_{l}\left[A_{l} T_{l}(0) \int_{0}^{2 \pi} \sin n \phi \cos l \phi d \phi\right. \\
\left.+B_{l} T_{l}(0) \int_{0}^{2 \pi} \sin n \phi \sin l \phi d \phi\right] \exp \left(-l^{2} D_{r} t\right)
\end{array}
$$


Usando as propriedades de ortogonalidade do seno e cosseno

$$
\begin{aligned}
& \int_{0}^{2 \pi} \cos n \phi \cos m \phi d \phi=\epsilon \pi \delta_{n, m} \\
& \int_{0}^{2 \pi} \sin n \phi \sin m \phi d \phi=\pi \delta_{n, m} \\
& \int_{0}^{2 \pi} \sin n \phi \cos m \phi d \phi=0
\end{aligned}
$$

ficamos com a equação (4.46)

$$
\int_{0}^{2 \pi} \frac{\cos n \phi G(\phi, t) d \phi}{\pi \epsilon}=A_{n} T_{n}(0)
$$

e com a equação (4.47)

$$
\int_{0}^{2 \pi} \frac{\sin n \phi G(\phi, t) d \phi}{\pi}=B_{n} T_{n}(0)
$$

Definimos $\phi(0)=\phi_{0}$, então

$$
G\left(\phi_{0}, 0\right)=\delta\left(\phi_{0}\right)
$$

portanto as equações (4.51) e (4.52) ficam

$$
A_{n} T_{n}(0)=\frac{\cos n \phi_{0}}{\pi \epsilon}, \quad B_{n} T_{n}(0)=\frac{\sin n \phi_{0}}{\pi}
$$

A solução da equação da difusão no plano fica

$$
G\left(\phi, t ; \phi_{0}, 0\right)=\sum_{l}\left[\frac{\cos l \phi_{0} \cos l \phi}{\pi \epsilon}+\frac{\sin l \phi_{0} \sin l \phi}{\pi}\right] \exp \left(-l^{2} D_{r} t\right)
$$

\subsubsection{Difusão rotacional no cone}

Neste modelo, o dipolo associado á molécula pode difundir livremente dentro de um cone, com ângulo polar máximo $\theta=\theta_{\max }$. Sua simetria é dada com relação ao eixo $z$.

A solução da equação (4.19) é um polinômio de legendre associado de grau $l_{n}^{m}$ e ordem $m$. A constante $l_{n}^{m}$ é a sequência de valores $l$ que satisfaz a condição de contorno para um dado valor de $m$. O índice $n$ é definido para que $l_{1}^{m}<l_{2}^{m}<l_{3}^{m}<\ldots$ para algum $m$. Como estamos tratando da rotação restrita em um cone, o ângulo polar está variando de $0<\theta<\theta_{\max }$, 


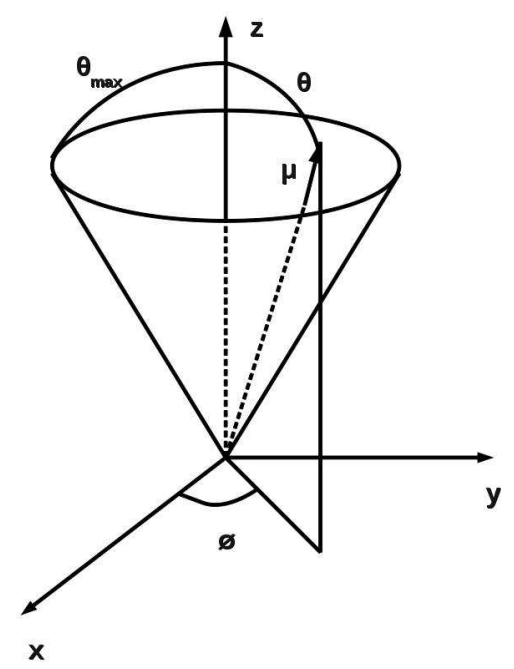

Figura 4.3: Dipolo associado à molécula difundindo livremente dentro de um cone

sendo $\theta_{\max }$ o ângulo máximo (abertura máxima do cone). O ângulo azimutal fica no intervalo $0 \leq \phi \leq 2 \pi$

A solução da equação (4.19) é um polinômio de Legendre associado dado por $P_{l_{n}^{m}}^{m}(x)=(-1)^{m}\left(1-x^{2}\right)^{m / 2}\left(-\frac{1}{2}\right)^{m}\left\{\prod_{k=1}^{m} \frac{\left(-l_{n}^{m}+k-1\right)\left(l_{n}^{m}+k\right)}{k}\right\}{ }_{2} F_{1}\left(-l_{n}^{m}+m, l_{n}^{m}+1+m ; 1+m ; z\right)$

onde $z=(1-x) / 2$ e $x=\cos (\theta)$ [19]. A função ${ }_{2} F_{1}\left(-l_{n}^{m}+m, l_{n}^{m}+1+m ; 1+m ; z\right)$ é uma função hipergeométrica definida por

$$
{ }_{2} F_{1}\left(1+m+l_{n}^{m}, m-l_{n}^{m}, 1+m ; \frac{1}{2}(1-\cos \theta)\right)=\sum_{k=0}^{\infty} \frac{\left(1+m+l_{n}^{m}\right)_{k}\left(m-l_{n}^{m}\right)_{k}}{k !(1+m)_{k}} \sin ^{2 k}\left(\frac{\theta}{2}\right) .
$$

Substituindo as soluções das equações (4.12), (4.14) e (4.56) na equação (4.5) obtemos

$$
G(\theta, \phi, t)=\sum_{n=1}^{\infty} \sum_{m=0}^{\infty} \exp \left[-l_{n}^{m}\left(l_{n}^{m}+1\right) D_{r} t\right]\left[A_{n}^{m} \cos m \phi+B_{n}^{m} \sin m \phi\right] P_{l_{n}^{m}}^{m}(\cos \theta)
$$

que é a solução da equação de difusão para o movimento restrito ao cone.

A condição de contorno do problema é

$$
\left.\frac{\partial G(\Omega, t)}{\partial \theta}\right|_{\theta=\theta_{\max }}=0
$$

Vamos definir $\lambda_{n, m}=l_{n}^{m}\left(l_{n}^{m}+1\right)$ para facilitar a abertura das somatórias da equação 4.58 
e observar como a condição de contorno interfere nos demais termos da solução. Então

$$
\begin{aligned}
G & =\left\{\exp \left(-\lambda_{1,0} t\right) A_{1}^{0} P_{l_{1}^{0}}^{0}+\exp \left(-\lambda_{1,1} t\right)\left[A_{1}^{1} \cos \phi+B_{1}^{1} \sin \phi\right] P_{l_{1}^{1}}^{1}+\ldots\right\}+ \\
& +\left\{\exp \left(-\lambda_{2,0} t\right) A_{2}^{0} P_{l_{2}^{0}}^{0}+\exp \left(-\lambda_{2,1} t\right)\left[A_{2}^{1} \cos \phi+B_{2}^{1} \sin \phi\right] P_{l_{2}^{1}}^{1}+\ldots\right\}+ \\
& +\ldots \ldots \ldots . . .+\exp \left(-\lambda_{n, m} t\right)\left[A_{n}^{m} \cos m \phi+B_{n}^{m} \sin m \phi\right] P_{l_{n}^{m}}^{m}
\end{aligned}
$$

Se aplicarmos a condição de contorno da equação (4.59) na equação (4.58) é o mesmo que fazermos

$$
\left.\frac{d P_{l_{n}^{m}}^{m}(\cos \theta)}{d \cos \theta}\right|_{\cos \theta=\cos \theta_{\max }}=0 .
$$

Uma das propriedades do polinômio de Legendre associado é

$$
\int_{\mu_{0}}^{1} P_{l_{n}^{m}}^{m}(\mu) P_{l_{n^{\prime}}^{m}}^{m}(\mu) d \mu=H_{n}^{m} \delta_{n n^{\prime}}
$$

com

$$
\delta_{n n^{\prime}}=\left\{\begin{array}{lll}
1, & \text { se } & n=n^{\prime}, \\
0, & \text { se } & n \neq n^{\prime} .
\end{array}\right.
$$

$H_{n}^{m}$ é um fator de normalização.

Outras propriedades importantes e conhecidas são as propriedade de ortogonalidade do seno e cosseno

$$
\begin{aligned}
& \int_{0}^{2 \pi} \cos m \phi \cos m^{\prime} \phi d \phi= \begin{cases}\pi \delta_{m m^{\prime}} & m \neq 0 \\
2 \pi & m=0\end{cases} \\
& \int_{0}^{2 \pi} \sin m \phi \sin m^{\prime} \phi d \phi= \begin{cases}\pi \delta_{m m^{\prime}} & m \neq 0 \\
0 & m=0\end{cases} \\
& \int_{0}^{2 \pi} \sin m \phi \cos m^{\prime} \phi d \phi=0 \\
& \text { qualquer }
\end{aligned}
$$

Para as condições iniciais temos

$$
\begin{aligned}
G(\Omega, 0) & =\delta[\Omega(t)-\Omega(0)]=\delta[\cos \theta(t)-\cos \theta(0)] \delta[\phi(t)-\phi(0)] \\
& =\sum_{n=1}^{\infty} \sum_{m=0}^{\infty}\left(A_{n}^{m} \cos m \phi+B_{n}^{m} \sin m \phi\right) P_{l_{n}^{m}}^{m}(\mu) .
\end{aligned}
$$


Com as propriedade do seno e cosseno e as condições iniciais, equação (4.64), podemos determinar as constantes $A_{n}^{m}$ e $B_{n}^{m}$

$$
\begin{aligned}
\int G(\Omega, 0) P_{l_{n^{\prime}}^{m}}^{m^{\prime}}(\mu) \cos m^{\prime} \phi d \Omega & =\int \delta[\Omega(t)-\Omega(0)] P_{l_{n^{\prime}}^{m \prime}}^{m^{\prime}}(\mu) \cos m^{\prime} \phi d \Omega \\
& =P_{l_{n^{\prime}}^{m^{\prime}}}^{m^{\prime}}(\mu(0)) \cos m^{\prime} \phi(0) .
\end{aligned}
$$

Mas ao mesmo tempo

$$
\begin{aligned}
& =\sum_{n=1}^{\infty} \sum_{m=0}^{\infty} \int_{\theta_{0}}^{\pi} \int_{0}^{2 \pi}\left(A_{n}^{m} \cos m \phi+B_{n}^{m} \sin m \phi\right) P_{l_{n}^{m}}^{m}(\cos \theta) P_{l_{n^{\prime}}^{m^{\prime}}}^{m^{\prime}}(\cos \theta) \cos m^{\prime} \phi \sin \theta d \theta d \phi= \\
& =\sum_{n=1}^{\infty} \sum_{m=0}^{\infty} \int_{\mu_{0}}^{1} P_{l_{n}^{m}}^{m} P_{l_{n^{\prime}}^{m^{\prime}}}^{m^{\prime}} d \mu\left\{\int_{0}^{2 \pi} A_{m}^{n} \cos m \phi \cos m^{\prime} \phi d \phi+B_{m}^{n} \sin m \phi \cos m^{\prime} \phi d \phi\right\}= \\
& =H_{n}^{m^{\prime}} A_{n}^{m^{\prime}} \pi \epsilon \quad \text { se } \begin{cases}m=0 & \text { se } \epsilon=2 ; \\
m \neq 0 & \text { se } \epsilon=1 .\end{cases}
\end{aligned}
$$

Esse $\epsilon$ é apenas um valor para respeitar a propriedade de ortogonalidade do cosseno. Com isso

$$
P_{l_{n}^{m}}^{m}(\mu(0)) \cos m \phi(0)=H_{n}^{m} A_{n}^{m} \pi \epsilon
$$

portanto

$$
A_{n}^{m}=\frac{\cos m \phi(0)}{\pi H_{n}^{m} \epsilon} P_{l_{n}^{m}}^{m}(\mu(0))
$$

Usaremos o mesmo raciocínio para determinar $B_{n}^{m}$

$$
\int G(\Omega, 0) P_{l_{n}^{m}}^{m} \sin m^{\prime} \phi d \Omega=P_{l_{n}^{m}}^{m}(\mu(0)) \sin m \phi(0)
$$

mas

$$
\begin{aligned}
& =\sum_{n=1}^{\infty} \sum_{m=0}^{\infty} \int_{0}^{\theta_{0}} \int_{0}^{2 \pi}\left(A_{n}^{m} \cos m \phi+B_{n}^{m} \sin m \phi\right) P_{l_{n}^{m}}^{m}(\cos \theta) P_{l_{n}^{m^{\prime}}}^{m^{\prime}}(\cos \theta) \sin m^{\prime} \phi \sin \theta d \theta d \phi \\
& =\sum_{n=1}^{\infty} \sum_{m=0}^{\infty} \int_{\mu_{0}}^{1} P_{l_{n}^{m}}^{m}(\mu) P_{l_{n}^{m^{\prime}}}^{m^{\prime}}(\mu) d \mu\left\{\int_{0}^{2 \pi} A_{n}^{m} \cos m \phi \sin m^{\prime} \phi d \phi+\int_{0}^{2 \pi} B_{n}^{m} \sin m \phi \sin m^{\prime} \phi d \phi\right\} \\
& =H_{n^{\prime}}^{m^{\prime}} \pi B_{n^{\prime}}^{m^{\prime}},
\end{aligned}
$$


portanto

$$
P_{l_{n}^{m}}^{m}(\mu(0)) \sin m \phi(0)=H_{n}^{m} B_{n}^{m} \pi
$$

e com isso

$$
B_{n}^{m}=\frac{\sin m \phi(0)}{\pi H_{n}^{m}} P_{l_{n}^{m}}^{m}(\mu(0))
$$

Conhecidos os termos $A_{n}^{m}$ e $B_{n}^{m}$, temos a seguinte densidade de probabilidade

$$
\begin{array}{r}
G\left(\theta, \phi, t ; \theta_{0}, \phi_{0}, 0\right)=\sum_{n=1}^{\infty} \sum_{m=0}^{\infty} \exp \left[-l_{n}^{m}\left(l_{n}^{m}+1\right) D_{r} t\right] \\
{\left[\frac{\cos m \phi(0)}{\pi H_{n}^{m} \epsilon} \cos m \phi+\frac{\sin m \phi(0)}{\pi H_{n}^{m}} \sin m \phi\right] P_{l_{n}^{m}}^{m}(\mu) P_{l_{n}^{m}}^{m}(\mu(0)) .}
\end{array}
$$

\subsection{Excitação do dipolo e anisotropia da fluorescência}

A energia absorvida na excitação do fluoróforo depende de sua orientação em relação à direção de polarização da luz, direção fixa no laboratório. Por outro lado, para investigar a anisotropia do meio, interessa medir a componente da luz emitida na direção paralela com à da luz polarizada.

No modelo de rotação livre, o referencial onde é resolvida a equação de difusão rotacional é coincidente com o referencial do laboratório.

Para os modelos em que o dipolo rotaciona no plano paralelo à superfície da esfera e para o modelo da rotação restrita ao cone, a equação de difusão rotacional é resolvida em um referencial fixo na superfície da esfera, com eixo $z$ paralelo ao vetor normal à superfície $\vec{n}$. Para que tenhamos as coordenadas do momento de dipolo com relação ao referencial do laboratório, referencial da polarização da luz, devemos fazer uma transformação de coordenadas. No primeiro caso, o momento de dipolo só tem componentes perpendiculares a $\vec{n}$. No caso da rotação restrita ao cone, há três componentes.

Então escrevemos as coordenadas do momento de dipolo

$$
\vec{\mu}=\left(\mu_{x}, \mu_{y}, \mu_{z}\right)_{\hat{n}},
$$

onde o índice $\hat{n}$ indica que as componentes estão dadas no referencial fixo na superfície da 

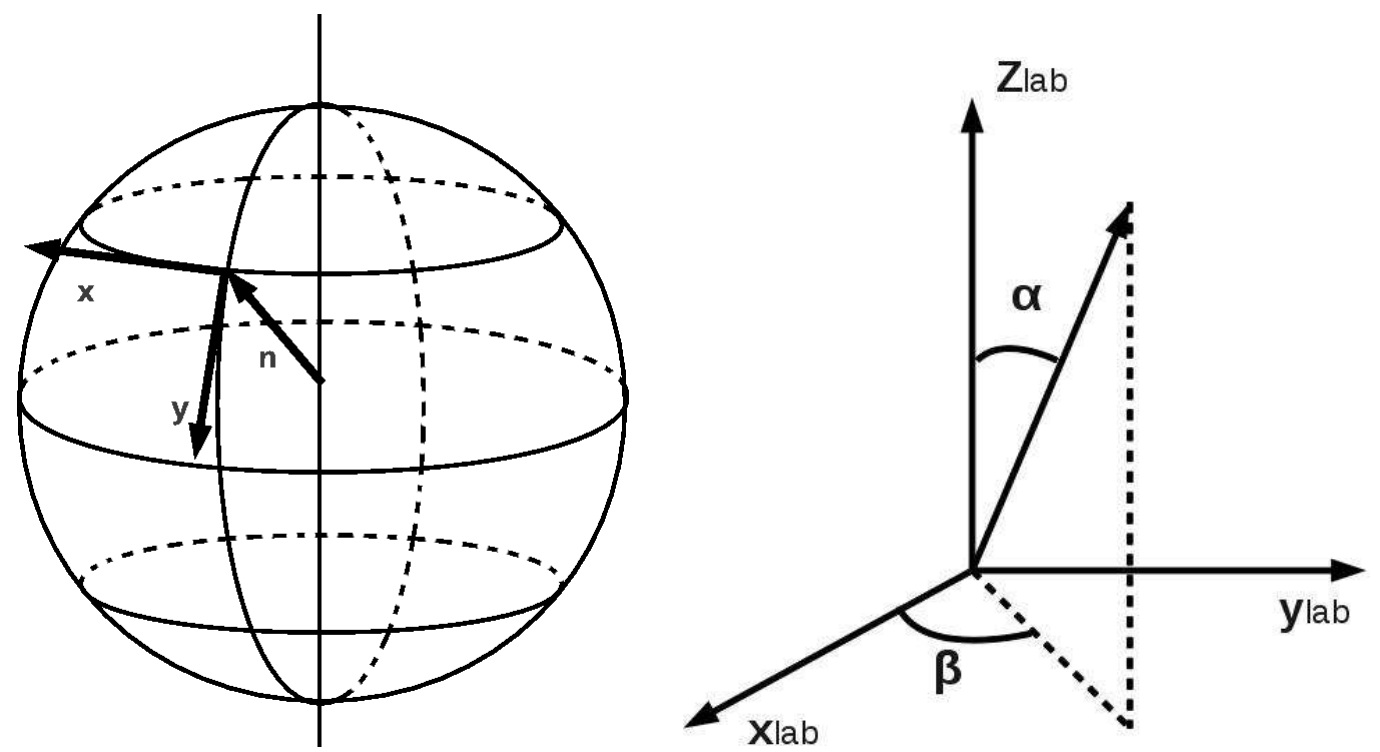

Figura 4.4: Direita - Escolha dos versores $\hat{x}$ e $\hat{y}$, tangentes à superfície da esfera. Esquerda Referencial do laboratório, onde está centrada a esfera.

esfera.

O cálculo da fração de dipolos excitados, bem como as componentes da intensidade da luz espalhada, referem-se à componente do momento de dipolo paralelo a um eixo do referencial do laboratório, que denominaremos $Z_{l a b}$. Assim, queremos obter as componentes de $\vec{\mu}$ no referencial fixo do laboratório, que representamos na forma

$$
\vec{\mu}=\left(\mu_{x}^{L}, \mu_{y}^{L}, \mu_{z}^{L}\right)_{L a b}
$$

Os eixos transversais são arbitrários tanto no referencial do laboratório quanto no referencial fixo na superfície da esfera. Assim, podemos utilizar a seguinte definição: dados os eixos fixos do laboratório, descrevemos o vetor $\vec{n}$ por meio das coordenadas esféricas $(\alpha, \beta)$. No referencial fixo na superfície da esfera, escolhemos os versores transversais com relação ao vetor $\hat{n}$, os versores $\hat{x}, \hat{y}$, da seguinte forma: $\hat{x}$ é tangente aos paralelos, com sentido antihorário em relação a $\hat{n}$, e $\hat{y}$ é tangente ao meridiano, sentido polo sul, conforme a figura 4.4 Vamos reescrever $\vec{\mu}$ no referencial $\vec{n}$, na forma

$$
\vec{\mu}=\mu_{x} \hat{x}+\mu_{y} \hat{y}+\mu_{z} \hat{z}
$$

e reescrever $\hat{x}, \hat{y}$ e $\hat{z}$ em termos das coordenadas do laboratório. A componente $\hat{z}$ é radial e 


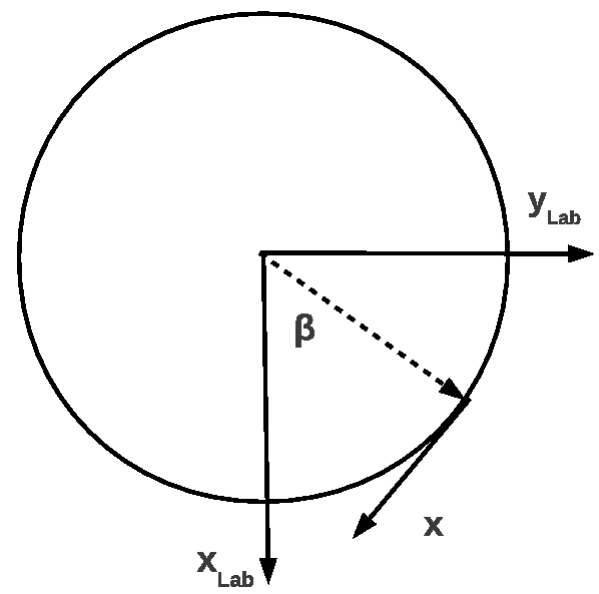

Figura 4.5: Versor $\hat{x}$ tangente aos paralelos, com sentido anti-horário em relação a $\hat{n}$

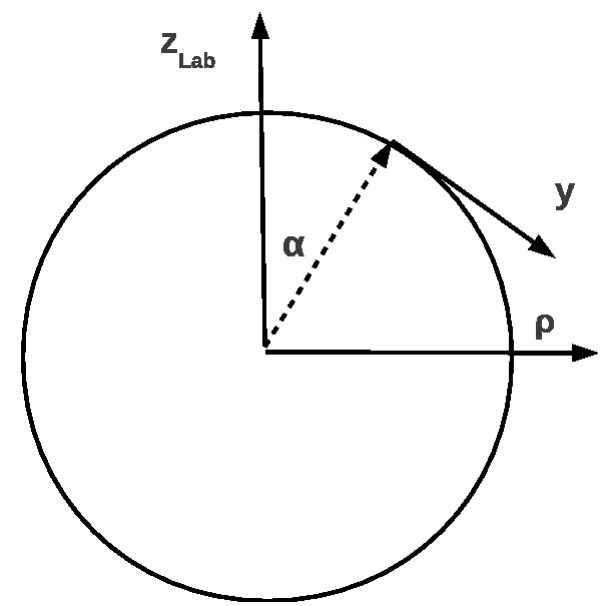

Figura 4.6: Versor $\hat{y}$ tangente ao meridiano

paralela a $\hat{n}$, portanto

$$
\hat{z}=\sin \alpha \cos \beta \hat{x}_{L a b}+\sin \alpha \sin \beta \hat{y}_{L a b}+\cos \alpha \hat{z}_{L a b} .
$$

A componente $\hat{x}$ é tangente ao paralelo e a análise da figura 4.5 mostra que podemos escrever

$$
\hat{x}=\sin \beta \hat{x}_{L a b}-\cos \beta \hat{y}_{L a b} .
$$

Finalmente, da figura 4.6, verificamos que

$$
\hat{y}=\cos \alpha \hat{\rho}-\sin \alpha \hat{z}_{L a b},
$$

onde

$$
\hat{\rho}=\cos \beta \hat{x}_{L a b}+\sin \beta \hat{y}_{L a b}
$$


portanto

$$
\hat{y}=\cos \alpha\left(\cos \beta \hat{x}_{L a b}+\sin \beta \hat{y}_{L a b}\right)-\sin \alpha \hat{z}_{L a b} .
$$

Se substituírmos as equações (4.77), (4.80) e (4.76) na equação (4.75), obtemos

$$
\begin{aligned}
\vec{\mu} & =\mu_{x}\left(\sin \beta \hat{x}_{L a b}-\cos \beta \hat{y}_{L a b}\right) \\
& +\mu_{y}\left[\cos \alpha\left(\cos \beta \hat{x}_{L a b}+\sin \beta \hat{y}_{L a b}\right)-\sin \alpha \hat{z}_{L a b}\right] \\
& +\mu_{z}\left[\sin \alpha \cos \beta \hat{x}_{L a b}+\sin \alpha \sin \beta \hat{y}_{L a b}+\cos \alpha \hat{z}_{L a b}\right]
\end{aligned}
$$

no referencial $\vec{n}$, temos, para o modelo de rotação no plano

$$
\vec{\mu}=\left(\mu_{x}, \mu_{y}, \mu_{z}\right)_{\hat{n}}=(\cos \phi, \sin \phi, 0)_{\hat{n}}
$$

e para o modelo do cone,

$$
\vec{\mu}=\left(\mu_{x}, \mu_{y}, \mu_{z}\right)_{\hat{n}}=(\sin \theta \cos \phi, \sin \theta \sin \phi, \cos \theta)_{\hat{n}}
$$

Basta agora utilizarmos a relação (4.82) para obter a componente paralela ao eixo $Z_{\text {Lab }}$ para calcular a probabilidade de excitação e a componente paralela à direção de polarização da luz incidente. Portanto, para rotação no plano temos

$$
\vec{\mu}=-\sin \phi \sin \alpha \hat{z}_{L a b}
$$

e, para o modelo do cone, temos

$$
\vec{\mu}=(-\sin \theta \sin \phi \sin \alpha+\cos \theta \cos \alpha) \hat{z}_{L a b}
$$

\subsection{Fluorescência de luz polarizada e anisotropia}

A probabilidade de excitação é

$$
\gamma(\Omega(0)) \propto \mu_{z}^{2}(t)
$$


no referencial do laboratório e consequentemente a emissão paralela à direção de polarização é

$$
I_{\|} \propto \mu_{z}^{2}(t)
$$

A distribuição de probabilidade $G\left(\Omega, t ; \Omega_{0}, 0\right)$ é a probabilidade do dipolo estar em uma orientação $\Omega$, que dependa da geometria do modelo, no instante $t$, dado que tinha uma distribuição inicial aleatória $\Omega_{0}$ no instante $t=0$. Então a fração de fluoróforos excitados, na orientação $\Omega_{0}$ no instante $t=0$ é

$$
f_{\text {exc }}(\Omega, t)=\int \gamma\left(\Omega_{0}, 0\right) G\left(\Omega, t ; \Omega_{0}, 0\right) d \Omega_{0}
$$

Devido ao fato de termos uma distribuição de momentos de dipolos, a componente de emissão paralela à polarização é descrita pela média, ou seja

$$
I_{||}=\left\langle\mu_{z}^{2}(t)\right\rangle=\int \mu_{z}^{2}(t) f_{\text {exc }}(\Omega, t) d \Omega
$$

\subsubsection{Rotação livre}

Integrando sobre todos $\theta_{0}$ possíveis para uma distribuição inicial aleatória, obtemos a fração de dipolos com orientação $\theta$ no instante $t$,

$$
f(\theta, t)=\int_{-1}^{1} \gamma\left(\theta_{0}\right) G\left(\theta, t ; \theta_{0}, 0\right) d\left(\cos \theta_{0}\right)
$$

que é a probabilidade conjunta.

Substituíndo $G\left(\theta, t ; \theta_{0}, 0\right)$ obtido na equação (4.72) e na equação (4.90), temos

$$
f(\theta, t)=\int_{-1}^{1} \gamma\left(\theta_{0}\right) \sum_{l} \frac{2 l+1}{2} P_{l}\left(\cos \theta_{0}\right) P_{l}(\cos \theta) \exp \left[-l(l+1) D_{r} t\right] d\left(\cos \theta_{0}\right) .
$$

Como a integral é sobre $\theta_{0}$,

$$
f(\theta, t)=\sum_{l} \frac{2 l+1}{2} P_{l}(\cos \theta) \exp \left[-l(l+1) D_{r} t\right] \int_{-1}^{1} \gamma\left(\theta_{0}\right) P_{l}\left(\cos \theta_{0}\right) d\left(\cos \theta_{0}\right) .
$$


Para uma distribuição inicial aleatória de ângulo $\theta_{0}$, a normalização de $\gamma\left(\theta_{0}\right)$ é dada por

$$
K \int_{-1}^{1} \cos ^{2} \theta_{0} d\left(\cos \theta_{0}\right)=1 \Longrightarrow K=\frac{3}{2}
$$

então

$$
\gamma\left(\theta_{0}\right)=\frac{3}{2} \cos ^{2} \theta_{0}
$$

O interessante é escrever essa probabilidade $f\left(\theta_{0}\right)$ como polinômios de Legendre, então

$$
\gamma\left(\theta_{0}\right)=P_{2}\left(\cos \theta_{0}\right)+\frac{1}{2}=P_{2}\left(\cos \theta_{0}\right)+\frac{1}{2} P_{0}\left(\cos \theta_{0}\right)
$$

É visível que os polinômios de Legendre relevantes são os de ordem 0 e 2, então a integral da equação (4.92) fica

$$
\int_{-1}^{1} \gamma\left(\theta_{0}\right) P_{l}\left(\cos \theta_{0}\right) d\left(\cos \theta_{0}\right)=\int_{-1}^{1}\left[\frac{1}{2} P_{0}\left(\cos \theta_{0}\right)+P_{2}\left(\cos \theta_{0}\right)\right] P_{l}\left(\cos \theta_{0}\right) d\left(\cos \theta_{0}\right)
$$

Resolvendo a equação (4.96) usando a ortogonalidade dos polinômios e substituindo o resultado na equação (4.92), temos

$$
f(\theta, t)=P_{2}(\cos \theta) \exp \left(-6 D_{r} t\right)+\frac{1}{2}
$$

que representa a probabilidade do momento de dipolo ser encontrado em uma configuração $\theta$ em $t$, dado que o momento de dipolo foi excitado e dada uma distribuição inicial aleatória.

Conhecida a probabilidade, equação (4.97), podemos obter as intensidades emitidas paralelamente e perpendicularmente com relação à direção de excitação. A intensidade paralela é dada por:

$$
\frac{I_{\|}}{I_{T}}=\int_{-1}^{1} f(\theta, t) \cos ^{2} \theta d(\cos \theta)=\frac{4}{15} \exp \left(-6 D_{r} t\right)+\frac{1}{3}
$$

A intensidade perpendicular é dada por:

$$
\frac{I_{\perp}}{I_{T}}=\int_{-1}^{1} f(\theta, t) \cos ^{2} \alpha d(\cos \theta)
$$


Como

$$
\begin{gathered}
\cos ^{2} \alpha+\cos ^{2} \beta+\cos ^{2} \theta=1 \text { e } \cos ^{2} \alpha=\cos ^{2} \beta, \\
\cos ^{2} \alpha=\frac{1}{2}-\frac{1}{2} \cos ^{2} \theta
\end{gathered}
$$

portanto,

$$
\frac{I_{\perp}}{I_{T}}=\int_{-1}^{1} f(\theta, t)\left(\frac{1}{2}-\frac{1}{2} \cos ^{2} \theta\right) d(\cos \theta)=\frac{1}{3}-\frac{2}{15} \exp \left(-6 D_{r} t\right) .
$$

A anisotropia de fluorescência é dada por:

$$
r(t)=\frac{I_{\perp}-I_{\|}}{I_{\perp}+2 I_{\|}}=\frac{I_{\perp}}{I_{T}}-\frac{I_{\|}}{I_{T}} .
$$

Substituindo os resultados obtidos para as intensidades nas equações acima, temos

$$
r(t)=0,4 \exp \left(-6 D_{r} t\right)
$$

onde $r(0)=0,4$ é a anisotropia inicial para $t=0$ e $r(\infty)=0$.

\subsubsection{Rotação no plano}

Da mesma forma que feito para a rotação livre, devemos determinar uma probabilidade conjunta entre a probabilidade do momento de dipolo ser excitado $\gamma\left(\alpha, \phi_{0}\right)$ e a probabilidade do momento de dipolo estar em uma configuração $\phi$ em $t$, dado que tenha uma orientação inicial $\phi_{0}$ em $t=0, G\left(\phi, t ; \phi_{0}, 0\right)$. Como descrito anteriormente, nesse modelo cada uma das moléculas é descrita por configurações $(\theta, \phi)$ no referencial fixo na vesícula e cada um desses referenciais possui configurações $(\alpha, \beta)$ com relação ao referencial do laboratório. Para uma distribuição aleatória de $\phi_{0}$, a probabilidade de encontrar o dipolo com orientação $\phi$ no tempo $t$, dado que rotaciona no plano perpendicular a $\vec{n}$, de ângulo azimutal $\alpha$ no referencial do laboratório, é dada por

$$
f(\phi, \alpha, t)=\int \gamma\left(\alpha, \phi_{0}\right) G\left(\phi, t ; \phi_{0}, 0\right) d \phi_{0}
$$


onde $G\left(\phi, t ; \phi_{0}, 0\right)$ é dado pela equação $(4.45)$.

A probabilidade do momento de dipolo ser excitado é proporcional ao quadrado da sua componente paralela com relação à direção de polarização da luz incidente, eixo $Z_{L a b}$. A componente paralela ao eixo $Z_{L a b}$ do dipolo localizado no ponto $(\alpha, \beta)$ da superfície da esfera é dada pela equação (4.84), portanto a probabilidade do dipolo ser excitado é

$$
\gamma\left(\alpha, \phi_{0}\right)=C \sin ^{2} \phi_{0} \sin ^{2} \alpha
$$

A normalização de $\gamma\left(\alpha, \phi_{0}\right)$, para uma distribuição aleatória de planos na esfera e uma distribuição aleatória de ângulos $\phi_{0}$ em $t=0$ dos dipolos nestes planos é dada por

$$
C \int_{0}^{\pi} \sin ^{2} \alpha \sin \alpha d \alpha \int_{0}^{2 \pi} \sin ^{2} \phi_{0} d \phi_{0}=1
$$

ficando

$$
C=\frac{3}{4 \pi}
$$

Portanto, a probabilidade de excitação para um dipolo de ângulo polar $\phi_{0}$, em um plano paralelo à superfície da esfera, é

$$
\gamma\left(\alpha, \phi_{0}\right)=\frac{3}{4 \pi} \sin ^{2} \alpha\left(\frac{1}{2}+\frac{1}{2} \cos 2 \phi_{0}\right)
$$

Devemos integrar com relação a $\phi_{0}$, supondo uma distribuição inicial aleatória dos dipolos nos planos. Substituindo as equações (4.55) e (4.109) na equação (4.105) temos

$$
\begin{gathered}
f(\alpha, \phi, t)=\int \frac{3}{4 \pi} \sin ^{2} \alpha\left(\frac{1}{2}+\frac{1}{2} \cos 2 \phi_{0}\right) G\left(\alpha, t ; \alpha_{0}, 0\right) d \phi_{0} \\
=\frac{3}{4 \pi} \sin ^{2} \alpha \int_{0}^{2 \pi}\left(\frac{1}{2}+\frac{1}{2} \cos 2 \phi_{0}\right) \sum_{l}\left[\frac{\cos l \phi_{0} \cos l \phi}{\pi \epsilon}+\frac{\sin l \phi_{0} \sin l \phi}{\pi}\right] \exp \left(-l^{2} D_{r} t\right) d \phi_{0} .
\end{gathered}
$$

Aplicando as propriedades de ortogonalidade dos senos e cossenos, observamos que apenas 
os termos $l=0$ e $l=2$ da somatória são relevantes. Com isso ficamos com

$$
f(\alpha, \phi, t)=\frac{3}{4 \pi} \sin ^{2} \alpha\left[\frac{1}{2}+\frac{1}{2} \cos 2 \phi \exp \left(-4 D_{r} t\right)\right]
$$

que é a probabilidade de se encontrar a molécula com a configuração $(\alpha, \phi)$ em $t$ dada a distribuição inicial aleatória.

A anisotropia é dada por

$$
r(t)=\frac{3}{2}\left\langle\left(\hat{z}_{L a b} \cdot \vec{\mu}\right)^{2}\right\rangle-\frac{1}{2}
$$

onde $\mu_{z}$ é dado pela equação (4.84) para o caso do dipolo rotacionando em um plano perpendicular à normal da superfície da esfera. Devemos integrar com relação a $\alpha$, o que corresponde a somar sobre a distribuição dos planos na superfície da vesícula

$$
\begin{gathered}
\left\langle\mu_{z}^{2}\right\rangle=\frac{3}{4 \pi} \int_{0}^{\pi} \frac{\sin ^{5} \alpha}{2} d \alpha \int_{0}^{2 \pi} \sin ^{2} \phi d \phi+ \\
+\frac{3}{4 \pi} \int_{0}^{\pi} \frac{\sin ^{5} \alpha}{2} d \alpha \int_{0}^{2 \pi} \cos 2 \phi \sin ^{2} \phi d \phi \exp \left(-4 D_{r} t\right) \\
=\frac{2}{5}+\frac{1}{5} \exp \left(-4 D_{r} t\right)
\end{gathered}
$$

então

$$
r(t)=\frac{3}{2}\left[\frac{2}{5}+\frac{1}{5} \exp \left(-4 D_{r} t\right)\right]-\frac{1}{2}
$$

e portanto a equação (4.113) fica

$$
r(t)=\frac{1}{10}+\frac{3}{10} \exp \left(-4 D_{r} t\right)
$$

Nesse caso, a anisotropia inicial também é $r(0)=0,4$ e devido a restrição do movimento do dipolo, a anisotropia não decai a zero com o tempo, $r(\infty)=0,1$.

\subsubsection{Rotação no cone}

Da mesma forma que foi feito nos outros dois modelos de rotação, podemos determinar uma probabilidade conjunta entre a probabilidade do momento de dipolo ser excitado $\gamma\left(\alpha, \theta_{0}, \phi_{0}\right)$ e a probabilidade do momento de dipolo estar em uma configuração $(\theta, \phi)$ em $t$, 
dado que tenha uma orientação $\left(\theta_{0}, \phi_{0}\right)$ em $t=0, G\left(\theta_{0}, \phi_{0}, 0 ; \theta, \phi, t\right)$. Para uma distribuição aleatória de $\theta_{0}$, obtemos

$$
f(\theta, \phi, \alpha, t)=\int \gamma\left(\alpha, \theta_{0}, \phi_{0}\right) G\left(\theta, \phi, t ; \theta_{0}, \phi_{0}, 0\right) \sin \theta_{0} d \phi_{0}
$$

A probabilidade do momento de dipolo ser excitado é proporcional ao quadrado da sua componente paralela com relação a direção de polarização da luz incidente, eixo $Z_{L a b}$. A componente paralela ao eixo $Z_{L a b}$ do dipolo localizado no ponto $(\alpha, \beta)$ da superfície da esfera é dada pela equação (4.85), portanto a probabilidade do dipolo ser excitado é

$$
\gamma\left(\alpha, \theta_{0}, \phi_{0}\right)=C\left(-\sin \theta_{0} \sin \phi_{0} \sin \alpha+\cos \alpha \cos \theta_{0}\right)^{2}
$$

A normalização de $\gamma\left(\alpha, \theta_{0}, \phi_{0}\right)$, para uma distribuição aleatória de cones na esfera e uma distribuição aleatória de ângulos $\left(\theta_{0}, \phi_{0}\right)$ em $t=0$ dos dipolos nestes planos é dada por

$$
C=\frac{3}{8 \pi^{2}\left(1-\cos \theta_{\max }\right)}
$$

Assim, a probabilidade da molécula ser excitada fica dada por

$$
\gamma\left(\alpha, \theta_{0}, \phi_{0}\right)=\frac{3}{8 \pi^{2}\left(1-\cos \theta_{\max }\right)}\left(-\sin \theta_{0} \sin \phi_{0} \sin \alpha+\cos \alpha \cos \theta_{0}\right)^{2}
$$

A anisotropia é dada por

$$
r(t)=\iiint \mu_{z}^{2}(t) f(\theta, \phi, \alpha, t) \sin \alpha d \alpha \sin \theta d \theta d \phi
$$

$\mathrm{ou}$

$$
\begin{array}{r}
r(t)=\iiint(-\sin \theta \sin \phi \sin \alpha+\cos \alpha \cos \theta)^{2} \gamma\left(\alpha, \theta_{0}, \phi_{0}\right) \\
G\left(\theta, \phi, t ; \theta_{0}, \phi_{0}, 0\right) d \Omega_{\hat{n}}(0) d \Omega_{\hat{n}}(t) d \Omega_{L a b}
\end{array}
$$


onde

$$
d \Omega_{\hat{n}}(0)=\sin \theta_{0} d \theta_{0} d \phi_{0}, \quad d \Omega_{\hat{n}}=\sin \theta d \theta d \phi \quad e \quad d \Omega_{L a b}=\sin \alpha d \alpha d \beta .
$$

A solução, de uma forma resumida, é dada por

$$
r(t)=r(0) \sum_{m=0}^{\infty} \sum_{n=1}^{\infty} A_{n}^{m} \exp \left(-\sigma_{n}^{m} D_{r} t\right)
$$

onde $A_{n}^{m}$ e $\sigma_{n}^{m}$ tem dependência em $m, n$ e $\theta_{\text {max }}$. A expressão completa para a anisotropia explicitando todas as integrais é apresentada no apêndice (D).

A única solução analítica possível é para $m=0$ e $n=1$, que corresponde ao comportamento da anisotropia para $t \rightarrow \infty$,

$$
\frac{r(t \rightarrow \infty)}{r(0)}=\left[\frac{1}{2} \cos \theta_{\max }\left(1+\cos \theta_{\max }\right)\right]^{2}
$$

O desenvolvimento completo dos cálculos para se chegar nessa expressão é apresentado também no apêndice (D).

\section{Solução numérica}

O uso das técnicas de solução numérica deve-se ao fato de não termos solução analítica para a condição de contorno da equação (4.61) e as integrais da equação (4.123).

A função de Legendre associado com coeficientes não inteiros que aparece na solução da equação de difusão rotacional para difusão no cone, bem como suas derivadas, tem um infinito número de raízes para um dado valor de $m$ e $\theta_{\max }$ fixo. A representação da função de Legendre associado pode ser feita através de uma série hipergeométrica

$$
P_{l_{n}^{m}}^{m}(\cos \theta)=\frac{(-1)^{m} \Gamma\left(m+l_{n}^{m}+1\right) \sin ^{m} \theta}{2^{m} m ! \Gamma\left(l_{n}^{m}-m+1\right)}{ }_{2} F_{1}\left(1+m+l_{n}^{m}, m-l_{n}^{m}, 1+m ; \frac{1}{2}(1-\cos \theta)\right)
$$

onde $n$ é a ordem dos $l^{\prime} s$ que irão satisfazer as condições impostas. A série hipergeométrica 
pode ser representada da seguinte forma

$$
{ }_{2} F_{1}(a, b, c ; z)=\sum_{k=0}^{\infty} \frac{(a)_{k}(b)_{k}}{k !(c)_{k}} z^{k}
$$

então

$$
{ }_{2} F_{1}\left(1+m+l_{n}^{m}, m-l_{n}^{m}, 1+m ; \frac{1}{2}(1-\cos \theta)\right)=\sum_{k=0}^{\infty} \frac{\left(1+m+l_{n}^{m}\right)_{k}\left(m-l_{n}^{m}\right)_{k}}{k !(1+m)_{k}} \sin ^{2 k}\left(\frac{\theta}{2}\right) .
$$

Fazendo alguns ajustes, podemos escrever a equação (4.129) como

$$
{ }_{2} F_{1}\left(1+m+l_{n}^{m}, m-l_{n}^{m}, 1+m ; \frac{1}{2}(1-\cos \theta)\right)=\sum_{k=0}^{\infty} \frac{\left(1+m+l_{n}^{m}\right)_{k}\left(m-l_{n}^{m}\right)_{k} m !}{k !(k+m) !} \sin ^{2 k}\left(\frac{\theta}{2}\right) .
$$

As raízes da equação (4.127) são determinadas se levarmos em conta que

$$
\sum_{k=0}^{\infty} \frac{\left(1+m+l_{n}^{m}\right)_{k}\left(m-l_{n}^{m}\right)_{k} m !}{k !(k+m) !} \sin ^{2 k}\left(\frac{\theta}{2}\right)=0
$$

pois os demais termos da equação (4.127) só serão nulos em dois casos, quando $\theta=0$ e $\theta=\pi$. Com isso determinaremos os $l^{\prime} s$ que são raízes de $P_{l_{n}^{m}}^{m}(\cos \theta)$ para um dado $\theta$ e $m$.

Para determinarmos as raízes da primeira derivada da função de Legendre associado

$$
\frac{d P_{l_{n}^{m}}^{m}(\cos \theta)}{d(\cos \theta)}=0
$$

usamos a seguinte fórmula de recorrência

$$
\sin ^{2} \theta \frac{d P_{l_{n}^{m}}^{m}(\cos \theta)}{d(\cos \theta)}=\left(l_{n}^{m}+m\right) P_{l_{n}^{m}-1}^{m}(\cos \theta)-l_{n}^{m} \cos \theta P_{l_{n}^{m}}^{m}(\cos \theta),
$$

então

$$
\frac{d P_{l_{n}^{m}}^{m}(\cos \theta)}{d(\cos \theta)}=\frac{1}{\sin ^{2} \theta}\left[\left(l_{n}^{m}+m\right) P_{l_{n}^{m}-1}^{m}(\cos \theta)-l_{n}^{m} \cos \theta P_{l_{n}^{m}}^{m}(\cos \theta)\right]=0
$$


Se observarmos a função

$$
P_{l_{n}^{m}-1}^{m}(\cos \theta)=\frac{(-1)^{m} \Gamma\left(m+l_{n}^{m}\right) \sin ^{m} \theta}{2^{m} m ! \Gamma\left(l_{n}^{m}-m\right)}{ }_{2} F_{1}\left(m+l_{n}^{m}, m-l_{n}^{m}+1,1+m ; \frac{1}{2}(1-\cos \theta)\right)
$$

podemos usar uma das propriedades da função gama e deixa-las com o seguinte formato

$$
\Gamma(z+1)=z \Gamma(z)
$$

então as funções gama que aparecem na equação (4.135) podem ser escritas como

$$
\begin{array}{r}
\Gamma\left(m+l_{n}^{m}+1\right)=\left(m+l_{n}^{m}\right) \Gamma\left(m+l_{n}^{m}\right) \\
\Gamma\left(m+l_{n}^{m}\right)=\frac{\Gamma\left(m+l_{n}^{m}+1\right)}{\left(m+l_{n}^{m}\right)} .
\end{array}
$$

e

$$
\begin{array}{r}
\Gamma\left(l_{n}^{m}-m+1\right)=\left(l_{n}^{m}-m\right) \Gamma\left(l_{n}^{m}-m\right) \\
\Gamma\left(l_{n}^{m}-m\right)=\frac{\Gamma\left(l_{n}^{m}-m+1\right)}{\left(l_{n}^{m}-m\right)} .
\end{array}
$$

Substituindo a equação (4.137), (4.138) na equação (4.135) temos

$$
P_{l_{n}^{m}-1}^{m}(\cos \theta)=\frac{(-1)^{m}\left(l_{n}^{m}-m\right) \Gamma\left(m+l_{n}^{m}+1\right) \sin ^{m} \theta}{2^{m} m !\left(m+l_{n}^{m}\right) \Gamma\left(l_{n}^{m}-m+1\right)}{ }_{2} F_{1}\left(m+l_{n}^{m}, m-l_{n}^{m}+1,1+m ; \frac{1}{2}(1-\cos \theta)\right) .
$$

Com isso podemos substituir as equações (4.127), (4.139) em (4.134) e obtermos

$$
\begin{array}{r}
\frac{1}{\sin ^{2} \theta} \frac{(-1)^{m} \Gamma\left(m+l_{n}^{m}+1\right) \sin ^{m} \theta}{2^{m} m !\left(m+l_{n}^{m}\right) \Gamma\left(l_{n}^{m}-m+1\right)}\left[\left(l_{n}^{m}-m\right)_{2} F_{1}\left(m+l_{n}^{m}, m-l_{n}^{m}+1,1+m ; \frac{1}{2}(1-\cos \theta)\right)\right. \\
\left.\left.-l_{n}^{m} \cos \theta_{2} F_{1}\left(1+m+l_{n}^{m}, m-l_{n}^{m}, 1+m ; \frac{1}{2}(1-\cos \theta)\right)\right] \neq 40140\right)
\end{array}
$$

O termo em evidência só será nulo no caso em que $\theta=0$ e $\theta=\pi$, portanto apenas o 
termo correspondente à diferença entre as funções hipergeométricas será nulo

$$
\begin{gathered}
\left(l_{n}^{m}-m\right) \sum_{k=0}^{\infty} \frac{\left(m+l_{n}^{m}\right)_{k}\left(m-l_{n}^{m}+1\right)_{k} m !}{k !(m+k) !} \sin ^{2 k}\left(\frac{\theta}{2}\right)- \\
-l_{n}^{m} \cos \theta \sum_{k=0}^{\infty} \frac{\left(m+l_{n}^{m}+1\right)_{k}\left(m-l_{n}^{m}\right)_{k} m !}{k !(m+k) !} \sin ^{2 k}\left(\frac{\theta}{2}\right)=0 .
\end{gathered}
$$

Fazendo alguns ajustes, chegamos a seguinte expressão para determinar as raízes da derivada

$$
\begin{array}{r}
\left(l_{n}^{m}-m-l_{n}^{m} \cos \theta\right)+\sum_{k=1}^{\infty} \frac{\left(m+l_{n}^{m}+1\right)_{k-1}\left(m-l_{n}^{m}+1\right)_{k-1} m !}{k !(m+k) !} \sin ^{2 k}\left(\frac{\theta}{2}\right) \\
\cdot\left[\left(l_{n}^{m 2}-m^{2}\right)\left(m-l_{n}^{m}+k\right)-l_{n}^{m}\left(m-l_{n}^{m}\right)\left(m+k+l_{n}^{m}\right) \cos \theta\right]=0
\end{array}
$$

O método numérico usado para determinar as raízes da função de Legendre associado, foi o método da secante. Para a obtenção dos coeficientes da equação (4.125) foi usado o método numérico de integração, conhecido como método do trapézio.

\subsection{Resultados da solução numérica}

Através do método da secante, obtivemos os valores das raízes de algumas funções de Legendre associados e suas derivadas, para $\theta_{\max }$ e $m$ fixo. Esses valores são apresentados no apêndice (B) e concordam com o apresentado em [20, 21].

Apesar de no apêndice (B) apresentarmos as raízes das funções de Legendere associados e suas derivadas, o que realmente interessa para o nosso trabalho são as raízes da derivada dos polinômios, já que essa é a condição de contorno. Para observar o comportamento dessas raízes que permite calcular os coeficientes das exponenciais, $\sigma_{n}^{m}$, na equação (4.125), fizemos o gráfico de $l_{n}^{m}$ em função do ângulo $\theta_{\max }$, para diferentes ordens de raízes e valores de $m$. O gráfico da figura 4.7 mostra que a primeira raiz para $m=0, l_{1}^{0}$ é nula para qualquer valor de $\theta_{\max }$. Mostra também que, para valores de $m$ e $n$ fixos, os valores das raízes diminuem com o aumento do valor de $\theta_{\max }$.

Conhecendo os valores das raízes da derivada dos polinômios de Legendre associado, usamos esses valores nas funções de Legendre associados da equação (4.123) e pelo método 


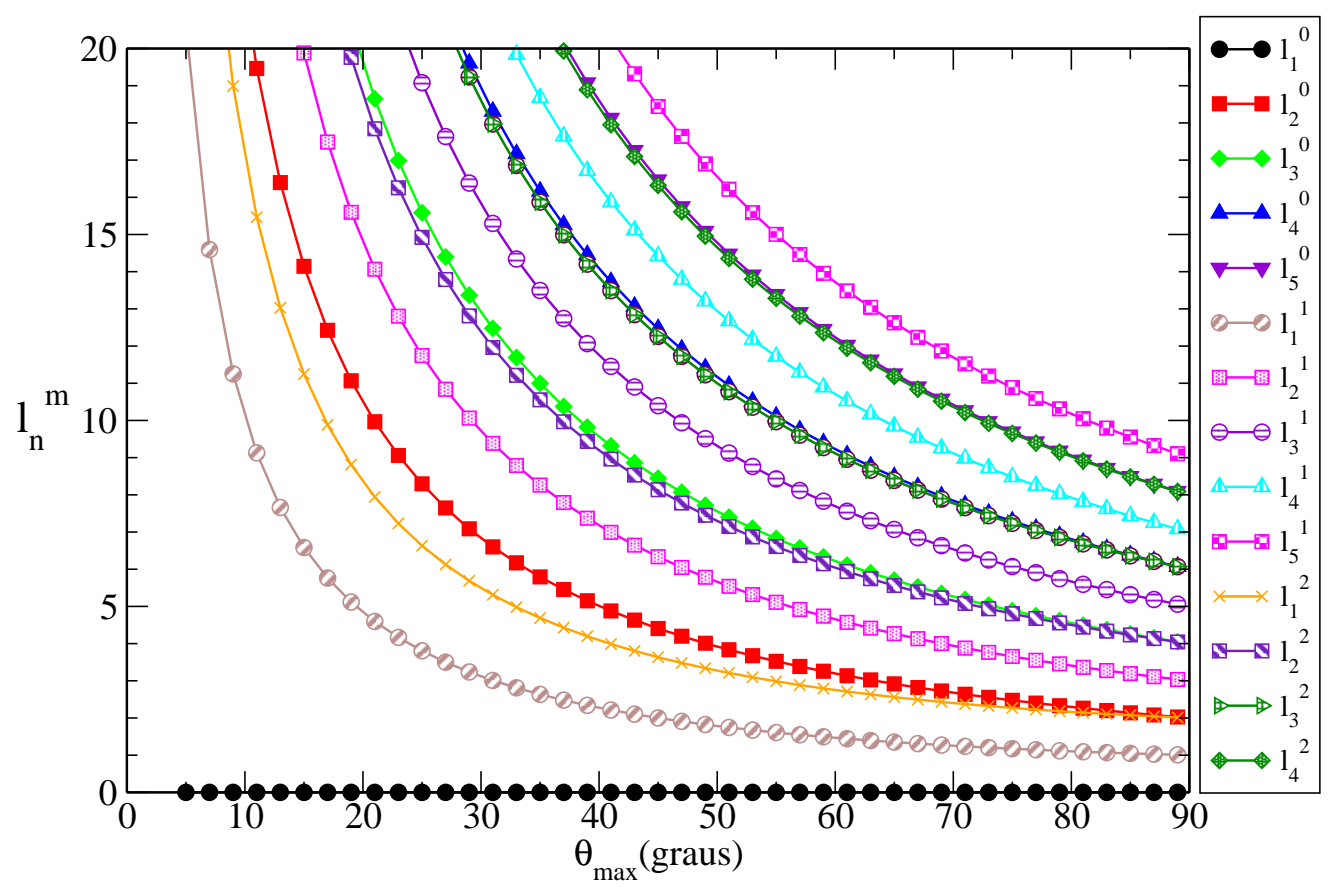

Figura 4.7: Raízes da derivada das funções de Legendre associados de coeficientes não inteiros. $\sigma_{n}^{m}$ são os coeficientes das exponenciais em (4.125)

de integração numérica calculamos os coeficientes $A_{n}^{m}$. O gráfico da figura 4.8 mostra esses coeficientes $A_{n}^{m}$ como função de $\theta_{\max }$. Podemos observar que a maioria dos coeficientes $A_{n}^{m}$ está muito próximo de zero. A figura 4.9 apresenta um detalhe da figura 4.8 e ilustra o comportamento dos coeficientes muito pequenos. Assim, dependendo da precisão que desejamos e do valor $\theta_{\max }$, apenas poucos coeficientes $A_{n}^{m}$ são relevantes na determinação da expressão teórica da anisotropia de fluorescência.

A partir dos valores das raízes da condição de contorno mostrado no gráfico da figura 4.7, podemos determinar os valores $\operatorname{dos} \sigma_{n}^{m}$, correspondentes para cada $l_{n}^{m}$. Esses coeficientes são apresentados no gráfico da figura 4.10

Conhecidos os valores dos coeficientes pré-exponenciais, figura 4.8, e os coeficientes da exponencial, figura 4.10, podemos determinar o gráfico da anisotropia de fluorescência levando em conta o $\theta_{\max }$ e os coeficientes relevantes. Por exemplo, para $\theta_{\max }<30^{\circ}$, uma excelente aproximação é

$$
r(t) \approx A_{1}^{0}+A_{1}^{1} \exp \left(-\sigma_{1}^{1} D_{r} t\right)
$$




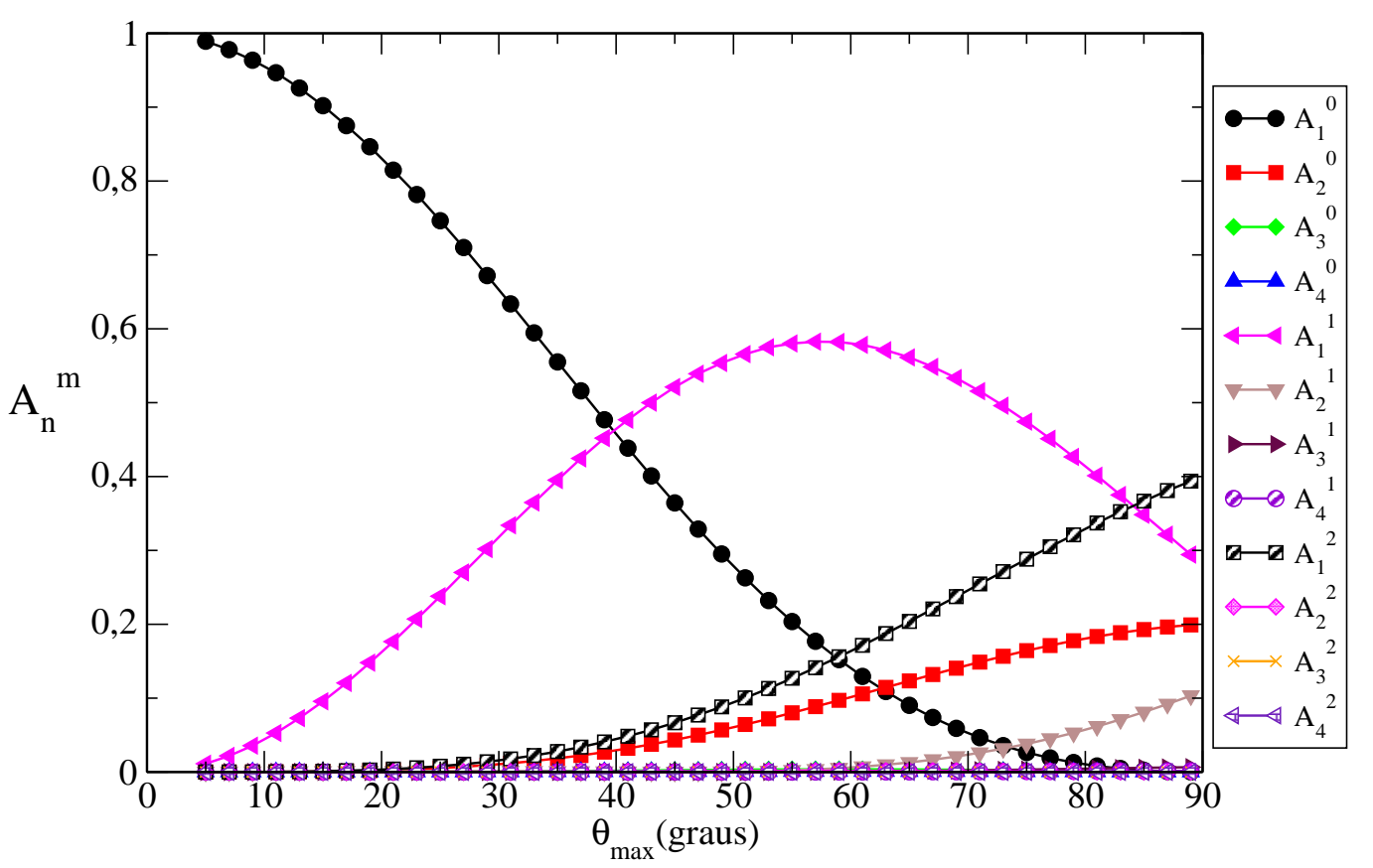

Figura 4.8: Coeficientes pré-exponenciais da solução da anisotropia para o Wobbling-cone

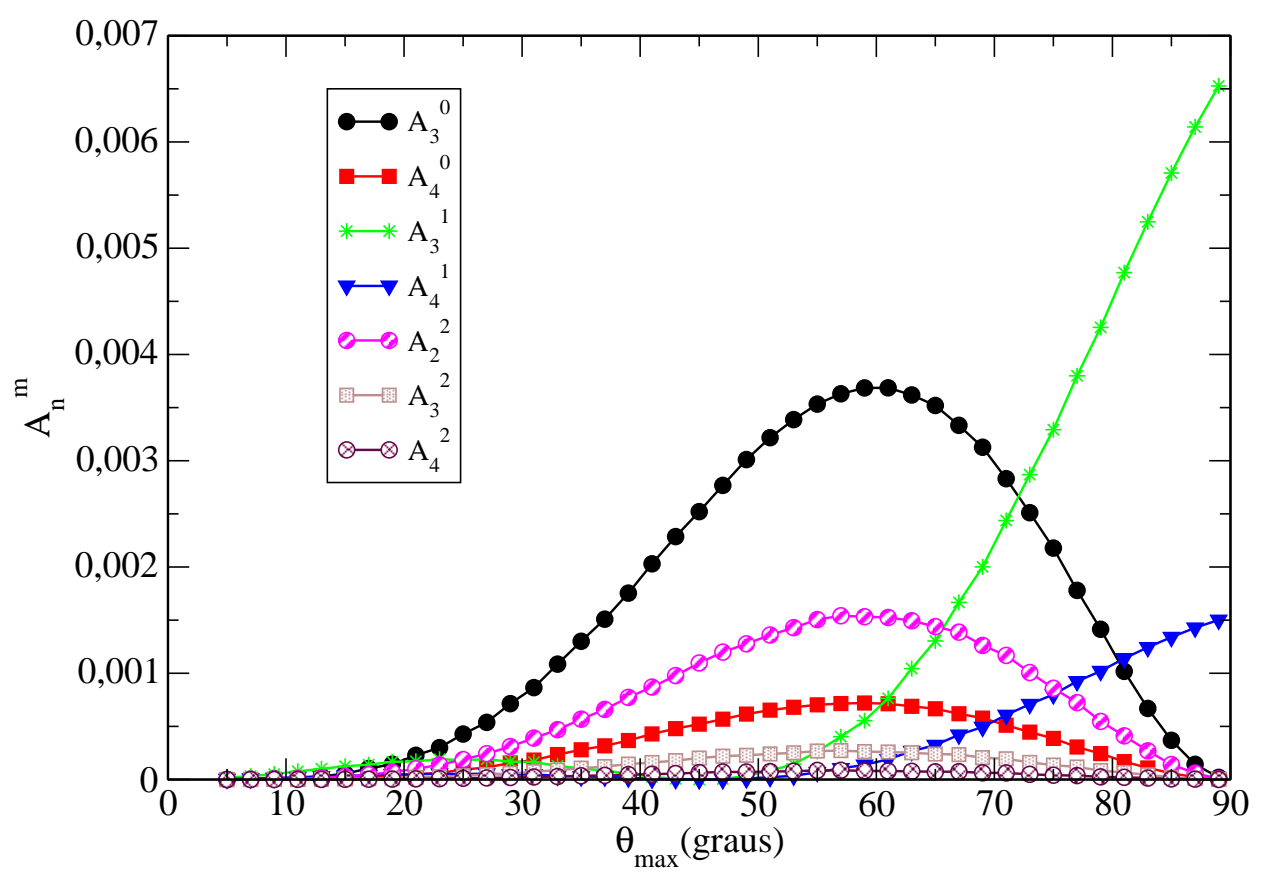

Figura 4.9: Coeficientes pré-exponenciais da solução da anisotropia para o Wobbling-cone, valores de $A_{n}^{m}$ que estão próximo de zero e não são visíveis no gráfico da figura 4.8 . 


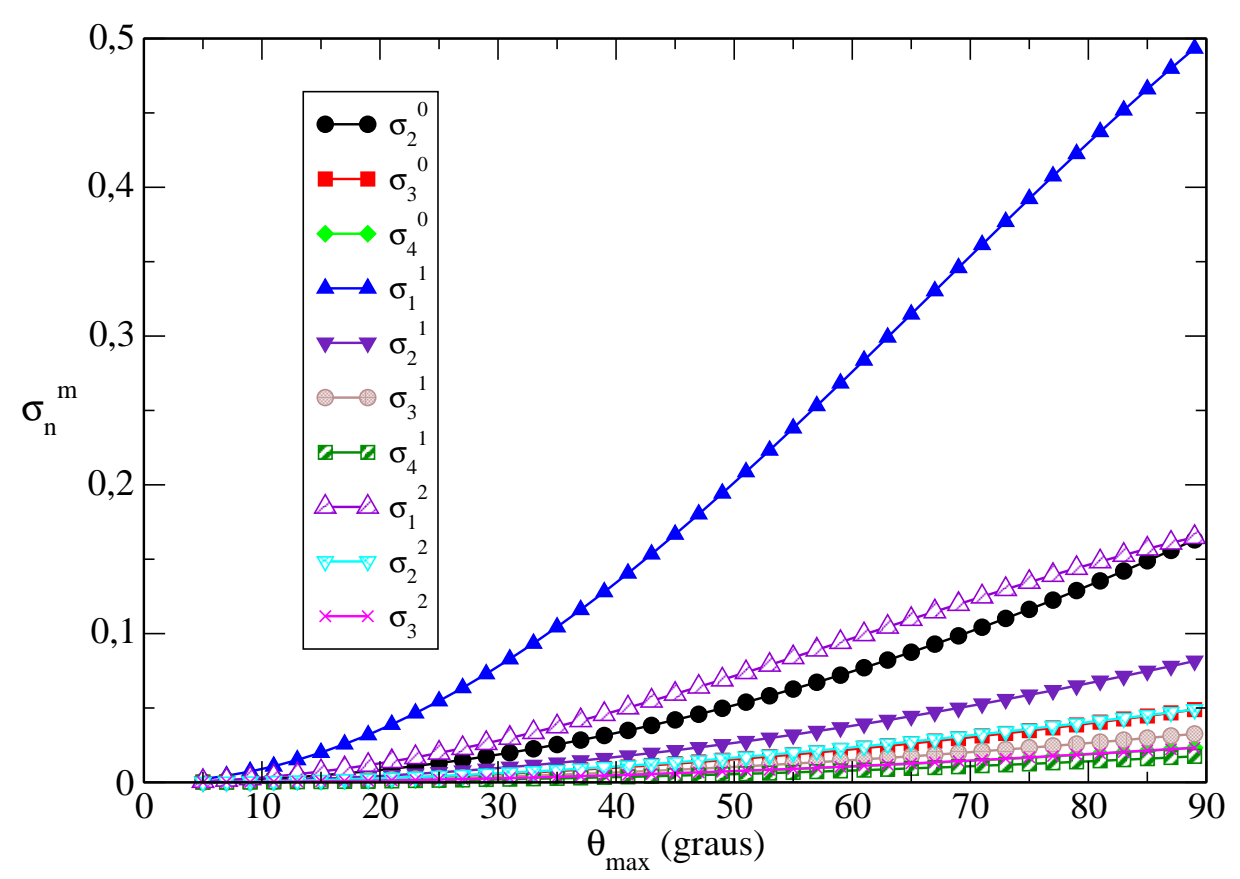

Figura 4.10: Valores dos fatores exponenciais para o wobbling-cone

e para $\theta_{\max }>50^{\circ}$

$$
r(t) \approx A_{1}^{0}+A_{1}^{1} \exp \left(-\sigma_{1}^{1} D_{r} t\right)+A_{1}^{2} \exp \left(-\sigma_{1}^{2} D_{r} t\right)
$$

A diferença entre os resultados destas aproximações e os resultados dos cálculos da expressão com até 100 termos na equação (4.125) não é visível na escala da figura. O gráfico da figura 4.11, mostra os valores numéricos para a anisotropia de fluorescência de moléculas com movimento restrito ao cone, para diferentes ângulos de restrição. 


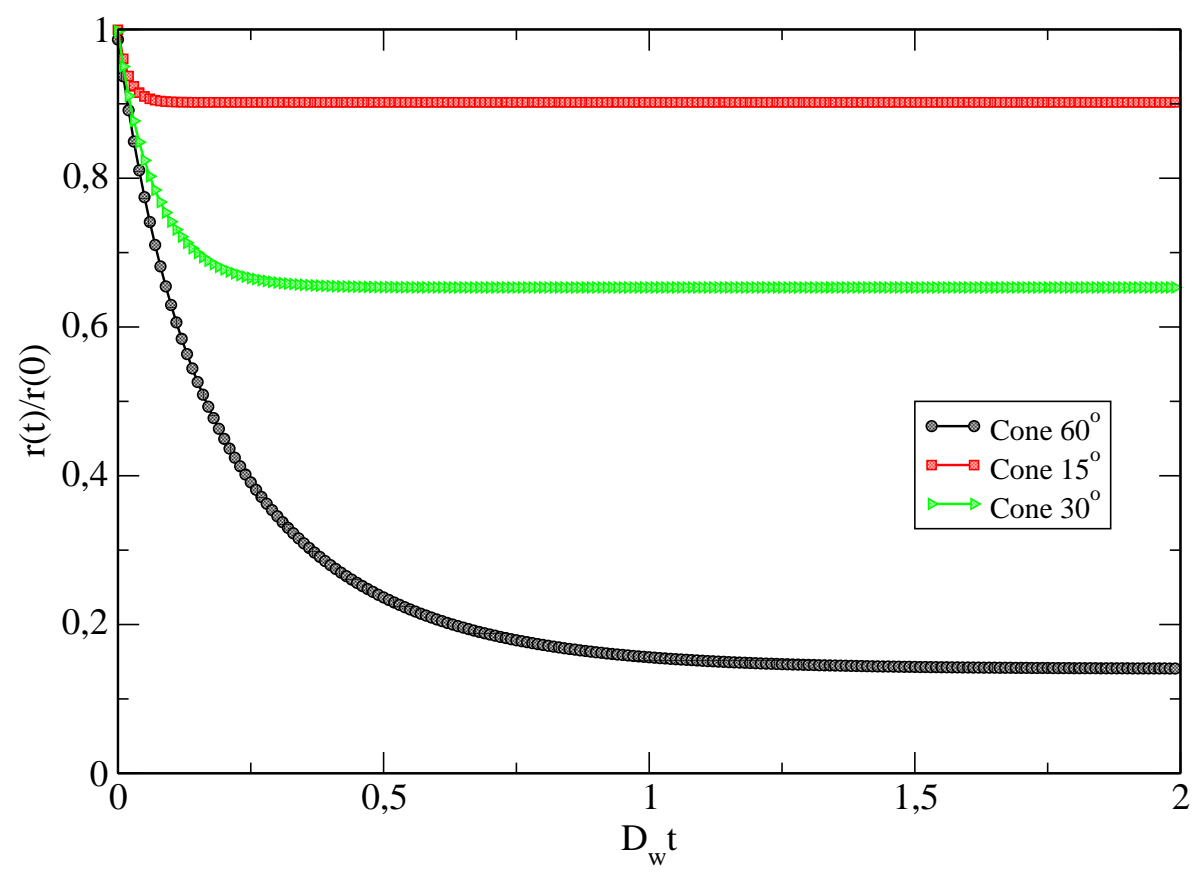

Figura 4.11: Anisotropia de fluorescência para $\theta_{\max }=15^{\circ}, \theta_{\max }=30^{\circ}$ e $\theta_{\max }=60^{\circ}$ 


\section{Capítulo 5}

\section{Difusão rotacional e anisotropia de}

\section{fluorescência - simulações numéricas}

A simulação de Monte Carlo é um dos recursos que pode ser utilizado na interpretação da anisotropia de fluorescência e é muito útil para casos em que não é possível fazer uma descrição analítica. Neste capítulo, vamos descrever a simulação de Monte Carlo utilizada, interpretação dos resultados obtidos e comparação com as expressões analíticas obtidas no capítulo anterior, para os três modelos propostos.

\subsection{Rotação livre}

No modelo de rotação livre, desejamos gerar uma distribuição inicial de dipolos com orientação aleatória $(\theta, \phi)$. Podemos descrever essa distribuição inicial em termos de uma distribuição em $\theta$, se levarmos em conta que a distribuição em $\phi$ resulta em uma densidade azimutal maior em $\theta=\frac{\pi}{2}$ do que em $\theta=0$. Utilizamos a simetria do sistema para fazer os movimentos aleatórios apenas em $\theta$. 


\subsubsection{Distribuição inicial}

A probabilidade do vetor dipolo $\vec{\mu}$ estar entre $\theta$ e $\theta+d \theta$ é dada pela razão entre a área do anel e a área da superfície da esfera, figura (5.1), então

$$
P(\theta) d \theta=\frac{R \sin \theta d \theta R d \phi}{R^{2} \int_{0}^{2 \pi} d \phi \int_{0}^{\pi} \sin \theta d \theta}=\frac{1}{2} \sin \theta d \theta .
$$

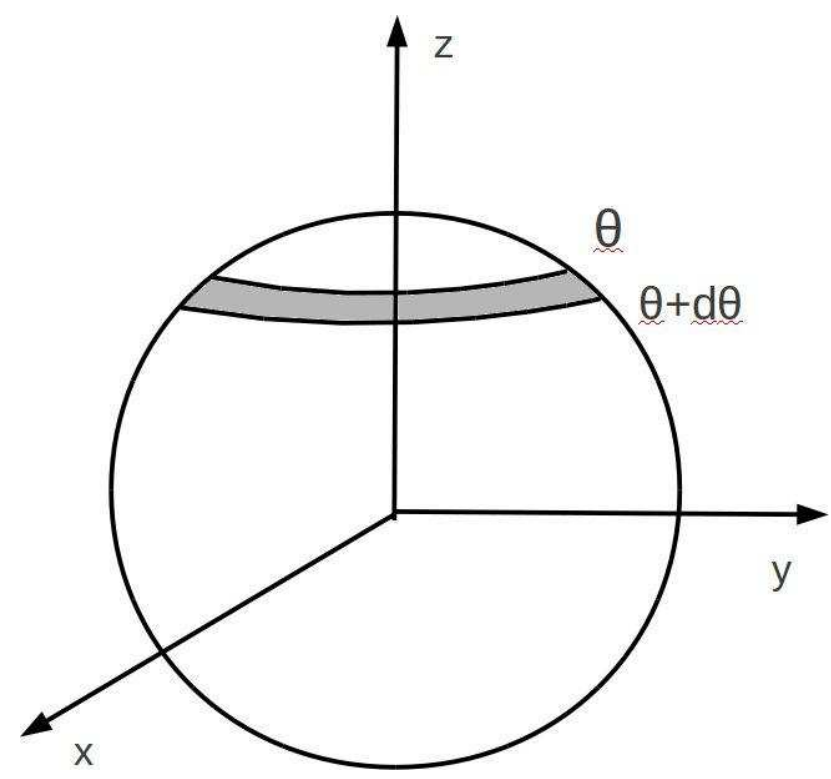

Figura 5.1: Anel da superfície da esfera para a determinação da distribuição em $\theta$

Conhecida a distribuição inicial aleatória de dipolos, desejamos saber quais desses dipolos serão excitados. A probabilidade do dipolo ser excitado é proporcional a $\cos ^{2} \theta$,

$$
\gamma\left(\theta_{0}\right)=C \cos ^{2} \theta_{0}
$$

que normalizada fica

$$
\gamma\left(\theta_{0}\right)=\frac{3}{2} \cos ^{2} \theta_{0}
$$

Queremos gerar esta distribuição através do sorteio de números aleatórios. O gerador de números aleatórios gera números reais distribuidos uniformemente entre 0 e 1 . Para conseguir a aleatoriedade, e ao mesmo tempo que a probabilidade seja satisfeita, devemos associar o ângulo $\theta$ a números aleatórios. Isso foi feito através do gerador de números aleatórios 
"ran2"[22], que gera números reais distribuídos uniformemente entre 0 e 1, porém, para fazer a relação entre a distribuição dos números aleatórios e o ângulo $\theta$, devemos fazer uma transformação [23].

Iniciamos considerando $P(x)$ a probabilidade do número aleatório distribuído uniformemente entre 0 e 1 ,

$$
P(x)=\left\{\begin{array}{cc}
1, & 0 \leq x<1 \\
0, & \text { fora }
\end{array}\right.
$$

Essa distribuição é normalizada

$$
\int_{-\infty}^{\infty} P(x) d x=\int_{0}^{1} 1 d x=1
$$

Nosso intuito é achar uma relação entre a variável aleatória $\theta$ para alguma distribuição de densidade de probabilidade $P(\theta)$, em termos de uma variável aleatória $x$ para uma distribuição uniforme.

A conservação da probabilidade desejada é

$$
|P(x) d x|=|P(\theta) d \theta|
$$

e portanto pode ser escrito

$$
\int_{x=-\infty}^{x=\infty} P(x) d x=\int_{\theta=-\infty}^{\theta=\infty} P(\theta) d \theta \quad \text { ou } \quad \int_{x=0}^{x} 1 d x=\int_{\theta=0}^{\theta} P(\theta) d \theta
$$

que fornece um resultado mais geral

$$
x=\int_{\theta=0}^{\theta} P(\theta) d \theta
$$

Para achar $\theta$, usamos a distribuição probabilidade $\gamma\left(\theta_{0}\right)$ da equação (5.3) na equação (5.8), sorteamos um número aleatório $x$ da distribuição uniforme e o valor de $\theta$ será o limite superior da integral da equação (5.8), que terá como resultado o valor do número aleatório sorteado. Assim obtivemos uma distribuição aleatória dos dipolos excitados no instante $t=0$. Isso foi feito até obtermos $10^{6}$ dipolos excitados. 


\subsubsection{Passeio aleatório}

Para a evolução dos dipolos, devemos novamente considerar o valor em $\phi$ de forma efetiva, levando em conta a simetria do problema para efetuar os movimentos aleatórios apenas em $\theta$. Com isso durante a relaxação do sistema, o ângulo $\theta$ entre o dipolo e a direção de polarização poderá aumentar, diminuir ou permanecer inalterado. Essa escolha é feita através de uma taxa de transição obtida através da equação mestra que governa a evolução temporal do ângulo $\theta$.

Seja $P(\theta, t)=P(\theta)$ a probabilidade de encontrar o sistema em uma configuração $\theta$ em um determinado instante de tempo $t$. Dessa forma temos

$$
\frac{\partial P(\theta, t)}{\partial t}=\sum_{\theta^{\prime}}\left[P\left(\theta^{\prime}\right) T\left(\theta^{\prime} \rightarrow \theta\right)-P(\theta) T\left(\theta \rightarrow \theta^{\prime}\right)\right]
$$

que é a equação mestra. Sendo que $T\left(\theta^{\prime} \rightarrow \theta\right)$ é a taxa de transição do ângulo azimutal da configuração $\theta^{\prime}$ para $\theta$ e $T\left(\theta \rightarrow \theta^{\prime}\right)$ é a taxa do modo inverso, em que o ângulo azimutal muda da configuração $\theta$ para $\theta^{\prime}$.

No estado estacionário, a probabilidade $P(\theta, t)$ não deve ser uma função explicita no tempo, ou seja

$$
\frac{\partial P(\theta, t)}{\partial t}=0
$$

Então uma condição suficiente para a condição de equilíbrio é o balanceamento detalhado, onde

$$
P\left(\theta^{\prime}\right) T\left(\theta^{\prime} \rightarrow \theta\right)=P(\theta) T\left(\theta \rightarrow \theta^{\prime}\right)
$$

para quaisquer configurações $\theta$ e $\theta^{\prime}$. Devemos ter o mesmo número de transições de $\theta$ para $\theta^{\prime}$ ou na direção contrária, de $\theta^{\prime}$ para $\theta$. Portanto temos

$$
\frac{T\left(\theta^{\prime} \rightarrow \theta\right)}{T\left(\theta \rightarrow \theta^{\prime}\right)}=\frac{P(\theta)}{P\left(\theta^{\prime}\right)}
$$

Essa probabilidade $P(\theta)$ é a mesma apresentada na equação (5.1).

A escolha de $\theta^{\prime}$ é

$$
\theta^{\prime}=\theta \pm \Delta \theta
$$


portanto a taxa de transição pode ser escrita como

$$
\frac{T(\theta \pm \Delta \theta \rightarrow \theta)}{T(\theta \rightarrow \theta \pm \Delta \theta)}=\frac{P(\theta)}{P(\theta \pm \Delta \theta)}=\frac{\sin \theta}{\sin (\theta \pm \Delta \theta)}
$$

Através da distribuição da equação (5.1) podemos observar que para o intervalo $0<$ $\theta \leq 90^{\circ}$ a probabilidade aumenta com o aumento de $\theta$ e para o intervalo $90^{\circ}<\theta \leq 180^{\circ}$ a probabilidade diminui com o aumento de $\theta$. Então devemos ter um balanceamento detalhado para cada um desses intervalos.

Para o caso em que $0 \leq \theta \leq 90^{\circ}$, escolhemos

$$
T(\theta-\Delta \theta \rightarrow \theta)=1
$$

$\mathrm{e}$

$$
T(\theta \rightarrow \theta-\Delta \theta)=\frac{\sin (\theta-\Delta \theta)}{\sin \theta}<1
$$

que satisfaz a condição (5.14).

Escolhemos um número aleatório e caso ele seja maior que 0,5, o ângulo aumentará, caso contrário escolhemos um número aleatório e comparamos com a taxa de transição da equação (5.16), se o número aleatório for menor que a taxa de transição o ângulo diminuirá, caso contário permanecerá com o mesmo valor.

Para o caso em que temos $90^{\circ}<\theta \leq 180^{\circ}$, impomos

$$
T(\theta \rightarrow \theta-\Delta \theta)=1
$$

e

$$
T(\theta-\Delta \theta \rightarrow \theta)=\frac{\sin \theta}{\sin (\theta-\Delta \theta)}<1
$$

que satisfaz a condição (5.14).

Escolhemos um número aleatório, e caso ele seja maior que 0,5, o ângulo diminuirá, caso contrário escolhemos um número aleatório e comparamos com a taxa de transição da equação (5.18), se o número aleatório for menor que a taxa de transição o ângulo aumentará, caso contrário permanecerá com o mesmo valor anterior. 


\subsubsection{Resultados}

Inicialmente, foram feitos alguns testes do algoritmo de simulação verificando se o comportamento médio de alguns parâmetros concordava com valores previstos teoricamente.

Para a difusão rotacional livre, é possível obter expressões analíticas para o ângulo médio $\langle\theta\rangle$, para o ângulo quadrático médio $\left\langle\theta^{2}\right\rangle$ e para a dispersão em função do tempo. Essas grandezas devem ser evoluídas no tempo de acordo com as expressões

$$
\begin{gathered}
\langle\theta\rangle=\frac{\pi}{2}, \\
\left\langle\theta^{2}\right\rangle=\frac{4}{9} \exp \left(-6 D_{r} t\right)+\frac{\pi^{2}}{2}-2, \\
\sigma^{2}=\frac{4}{9} \exp \left(-6 D_{r} t\right)+\frac{\pi^{2}}{4}-2,
\end{gathered}
$$

desenvolvidas no apêndice $\mathrm{A}$.

A figura 5.2 mostra o gráfico do comportamento do ângulo médio entre a direção de polarização $z$ e o vetor dipolo $\vec{\mu}$ ao longo da simulação. Podemos verificar que o ângulo

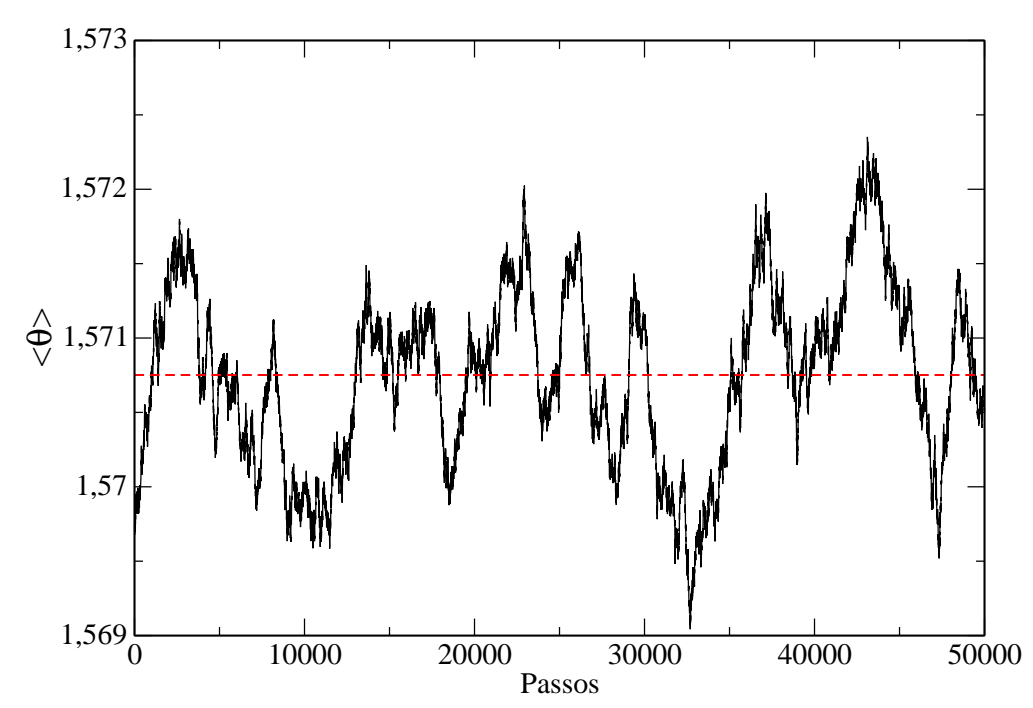

Figura 5.2: Média do ângulo entre o dipolo e a direção de polarização

medio $\langle\theta\rangle$, indicado pela linha pontilhada, concorda a expressão teórica.

Da mesma forma, $\left\langle\theta^{2}\right\rangle$ e $\sigma^{2}$, mostrados nas figuras $5.3(\mathrm{a})$ e $5.3(\mathrm{~b})$, convergem para os 
valores de equilíbrio previstos teoricamente.

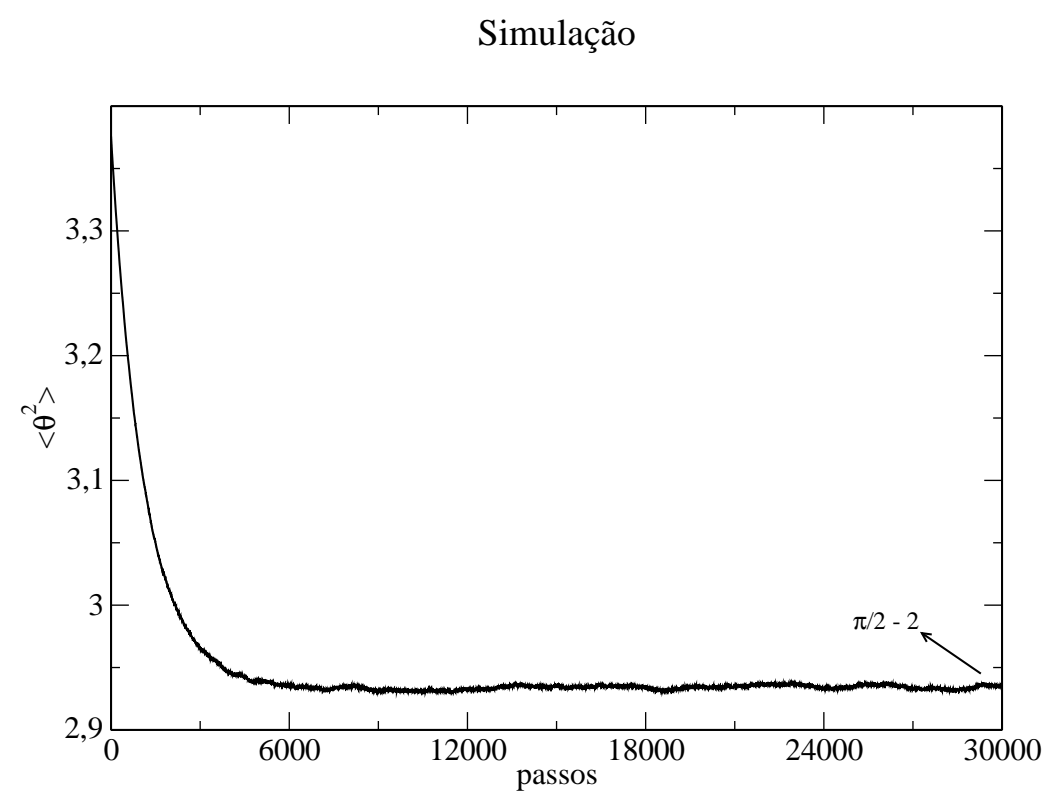

(a) Evolução do ângulo quadrático médio, $\left\langle\theta^{2}\right\rangle$, para a rotação livre

Simulação

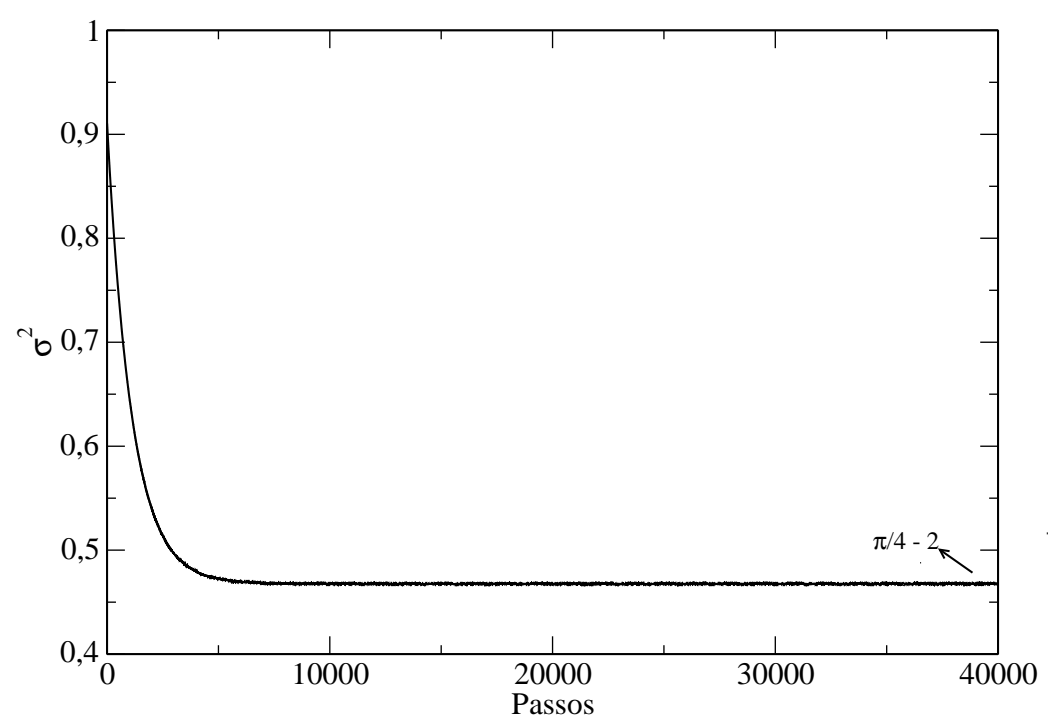

(b) Evolução da dispersão do ângulo entre a direção de polarização e o vetor dipolo

Figura 5.3

Apesar dos valores para $t=0$ e $t \rightarrow \infty$, dos gráficos da figura 5.3, concordarem com os valores analíticos apresentados nas equações (5.20) e (5.21), para que possamos fazer a comparação entre o resultado analítico e o simulado ao longo de toda a curva, é necessário saber o coeficiente de difusão da simulação. Esse coeficiente pode ser obtido através do 
ajuste dos dados iniciais a uma reta. O coeficiente de difusão é proporcional ao coeficiente angular do ajuste, e o termo de proporcionalidade é obtido através da expansão em Taylor das equações $(5.20)$ e $(5.21)$, para $t \approx 0$. As figuras 5.4(a) e 5.4(b), mostram os ajustes, para os dados iniciais da simulação, de $\left\langle\theta^{2}\right\rangle$ e $\sigma^{2}$, juntamente com os parâmetros do ajuste e o coeficiente de difusão correspondente.

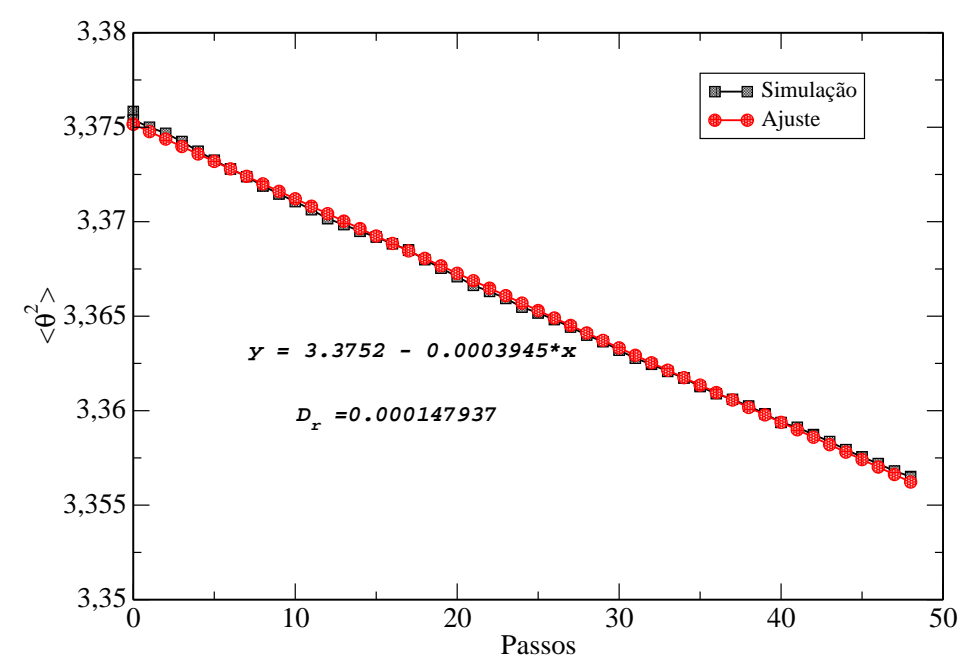

(a) Ajuste de reta dos dados iniciais da simulação de Monte Carlo do ângulo quadrado médio, para a rotação livre

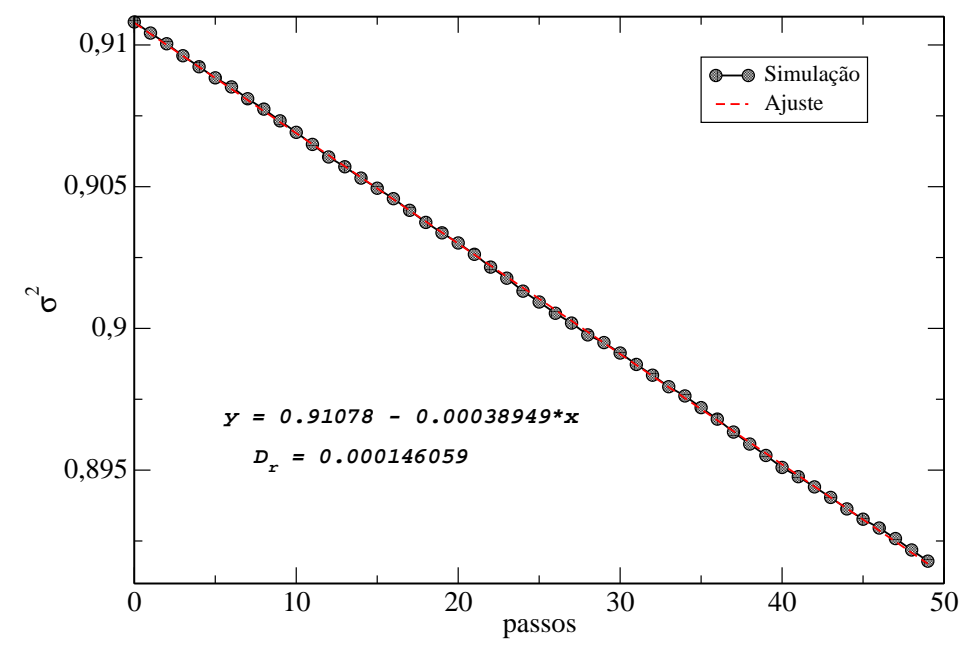

(b) Ajuste de reta dos dados iniciais da simulação de Monte Carlo da dispersão, para a rotação livre

Figura 5.4

Conhecidos os coeficientes de difusão, multiplicamos o eixo dos passos, dos gráficos da 
figura 5.3, pelo coeficiente e obtivemos o eixo em unidade adimensional. A comparação entre o resultado da simulação e a expressão analítica é apresentada na figura 5.5.

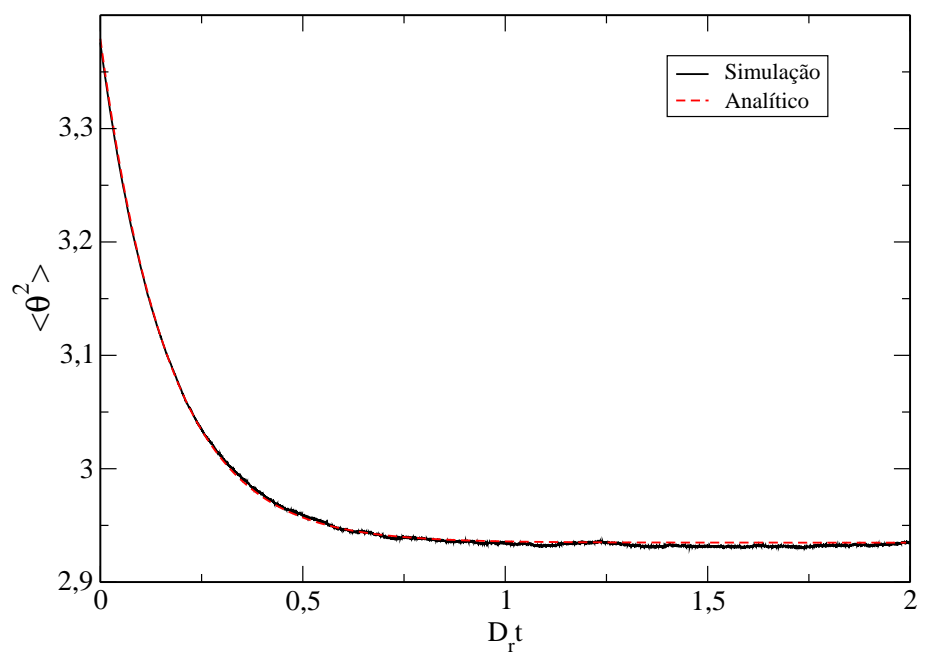

(a) Comparação entre os dados da simulação de Monte Carlo e o valor analítico, do ângulo quadrático médio $\left\langle\theta^{2}\right\rangle$ para dipolos rotacionando livremente

Dispersão Rotação Livre

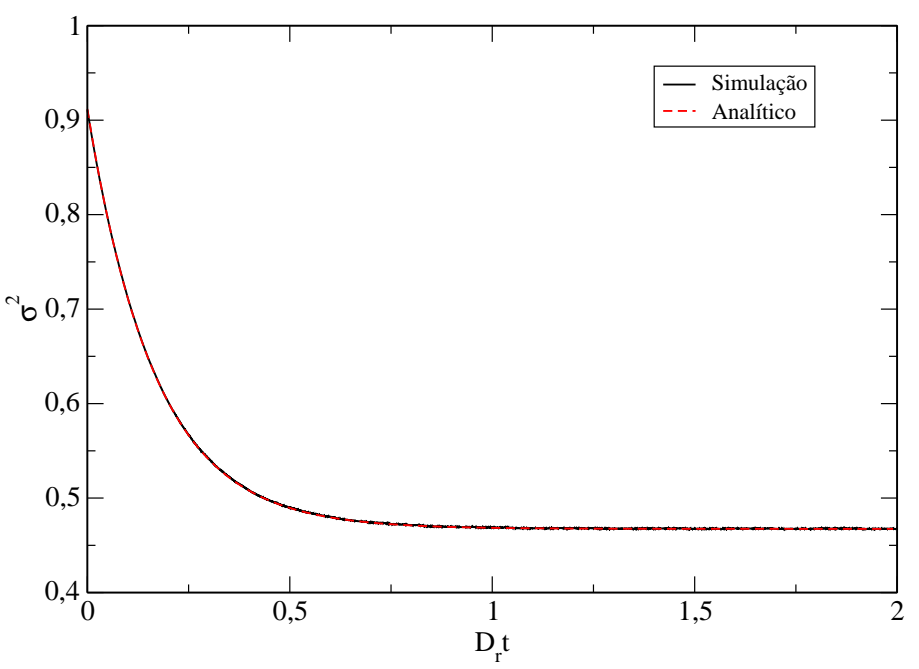

(b) Comparação entre os dados da simulação de Monte Carlo e o valor analítico, da dispersão para dipolos rotacionando livremente

Figura 5.5

A figura 5.5 mostra que os valores da simulação concordam com os valores analíticos, não somente em $t=0$ e $t \rightarrow \infty$, mas também ao longo da curva.

Um outro teste foi feito, para verificar se, a partir de uma orientação específica dos ângulos $\theta$, o sistema evoluiria para a distribuição aleatória, que respeita a distribuição da 
equação (5.1). A comparação entre a distribuição inicial aleatória dos ângulos $\theta$, a expressão (5.1) analítica, a evolução da distribuição final de ângulos a partir de uma orientação inicial dos dipolos em $\theta=0$, é feita na figura 5.6. A partir da figura (5.6), podemos observar que

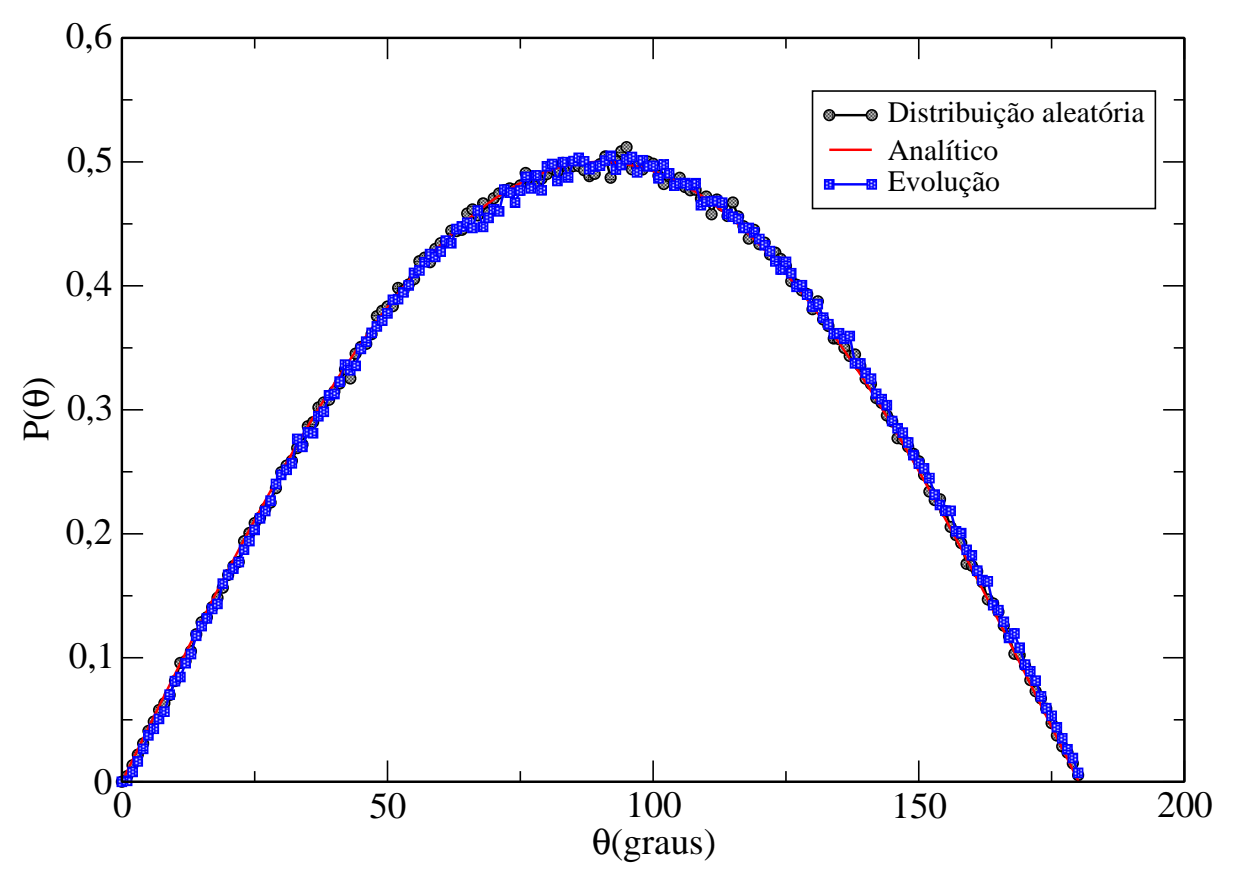

Figura 5.6: Comparação da distribuição inicial aleatória, com a expressão analítica 5.1 e a evolução da distribuição final a partir de uma orientação inicial dos dipolos em $\theta=0$

os ângulos $\theta$, iniciados em um valor específico, evoluem para a distribuição aleatória.

Com os testes do algoritmo de simulação bem estabelecidos, foi possível fazer a simulação da anisotropia de fluorescência. A figura (5.7) mostra o resultado da simulação de Monte Carlo e o ajuste, para a anisotropia de fluorescência de dipolos rotacionando livremente.

Os valores da simulação, para $t=0$ e $t=\infty$, do gráfico da figura (5.7), concordam com os valores analíticos apresentados na equação (4.104). Como já discutido anteriormente, para fazer a comparação entre o resultado analítico e o simulado ao longo de toda a curva, é necessário saber o coeficiente de difusão da simulação.

O coeficiente de difusão foi obtido através do ajuste dos dados da simulação, mostrado na figura (5.7), por uma função monoexponencial. O parâmetro da exponencial obtido no ajuste, foi dividido por 6, conforme a equação (4.104), e obtivemos o coeficiente de difusão 


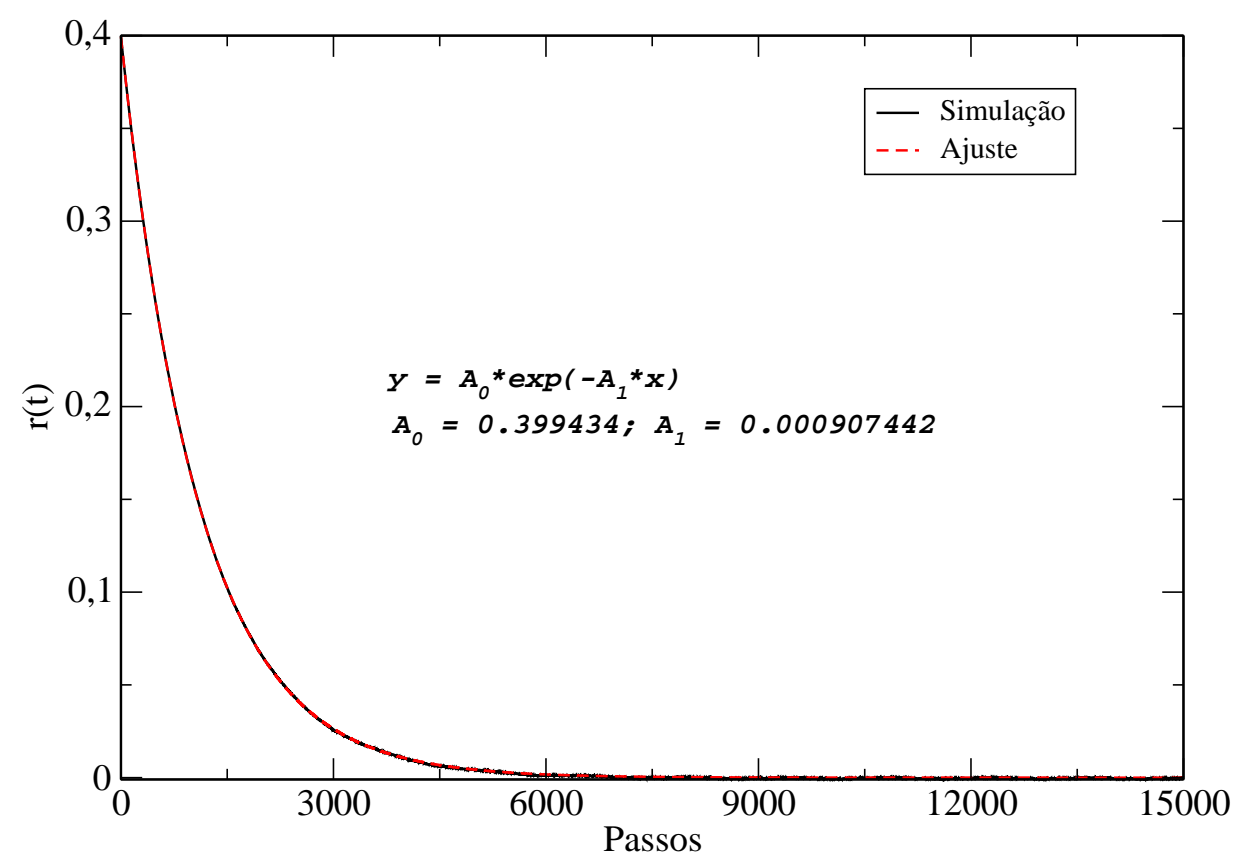

Figura 5.7: Simulação de Monte Carlo para anisotropia de fluorescência para dipolos rotacionando livremente e seu respectivo ajuste

$D_{r}=0,00015124$ passo $^{-1}$.

Conhecido o coeficiente de difusão podemos colocar no mesmo gráfico os dados analíticos e os simulados para verificar a concordância entre ambos. Multiplicamos o eixo dos passos da figura (5.7) pelo coeficiente de difusão obtidos e obtivemos o eixo em unidade adimensional. A figura (5.8) mostra essa comparação.

A figura (5.8) mostra que os valores da simulação concordam com os valores analíticos, não somente para $t=0$ e $t \rightarrow \infty$, como já haviamos observado anteriormente, mas também durante a relaxação do sistema.

\subsection{Rotação restrita}

Nos dois modelos de rotação restrita, o da rotação paralela à superfície da esfera e da rotação dentro do cone, os procedimentos para a determinação da distribuição inicial de dipolos são bastante semelhantes, na simulação. Por outro lado, o processo de difusão e de 


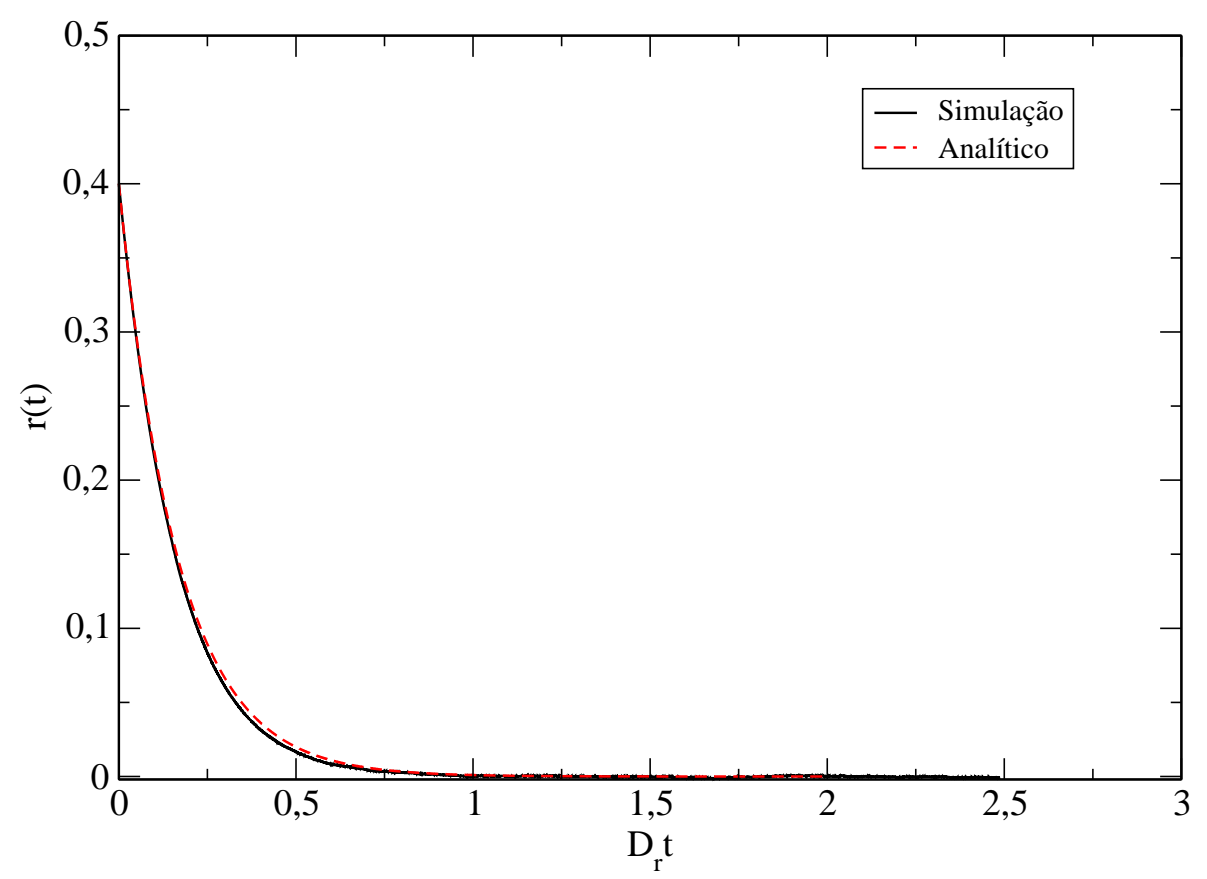

Figura 5.8: Comparação entre os valores analíticos e a simulação de Monte Carlo para anisotropia de fluorescência para dipolos rotacionando livremente

relaxação, são processos diferentes nas simulações e serão descritas separadamente a seguir.

Primeiro, selecionamos aleatoriamente vetores radiais $\hat{n}$, em seguida selecionamos aleatoriamente vetores perpendiculares aos vetores radiais, vetores $\vec{N}$. Os vetores dipolo são obtidos a partir da rotação, dos vetores radiais $\hat{n}$, usando como eixo de rotação o vetor $\vec{N}$. O ângulo de rotação envolvido leva em conta a geometria do modelo.

Por outro lado, o processo de difusão e de relaxação, são processos diferentes nas simulações e serão descritas separadamente a seguir.

\subsubsection{Distribuição inicial dos vetores dipolos}

Os algoritmos utilizados para gerar a distribuição inicial são inspirados no trabalho de Krishna et al. [8, 9].

Para a distribuição inicial dos dipolos, determinamos em primeiro lugar uma distribuição aleatória dos vetores radiais $\hat{n}$, vetores normais à superfície da esfera, que correspondem ao vetor do centro de massa do dipolo. As coordenadas dos vetores radiais $\hat{n}$ são dadas por 
$(\alpha, \beta)$, com $0 \leq \alpha \leq \pi$ e $0 \leq \beta \leq 2 \pi$, ângulos com relação ao referencial do laboratório (referencia figura 3.3a) estão fixos na vesícula.

A probabilidade para o ângulo $\alpha$ do vetor radial $\hat{n}$ é a mesma apresentada na equação (5.1), substituindo $\theta$ por $\alpha$. Usamos o mesmo raciocínio usado na rotação livre, para relacionar o ângulo $\alpha$ com números aleatórios. Para achar o ângulo $\alpha$, usamos a distribuição probabilidade $P(\alpha)$ da equação (5.1) na equação (5.8), sorteamos um número aleatório $x$ da distribuição uniforme, o valor de $\alpha$ será o limite superior da integral da equação (5.8), que terá como resultado o valor do número aleatório sorteado. A distribuição resultante já foi ilustrada na figura 5.6.

A coordenada $\beta$ do vetor radial $\hat{n}$ é obtida de uma forma mais simples, pelo fato de corresponder a uma distribuição uniforme, então basta apenas multiplicar o número aleatório por $2 \pi$. Na figura 5.9 ilustra o teste para a distribuição uniforme $P(\beta)=\frac{1}{2 \pi}$.

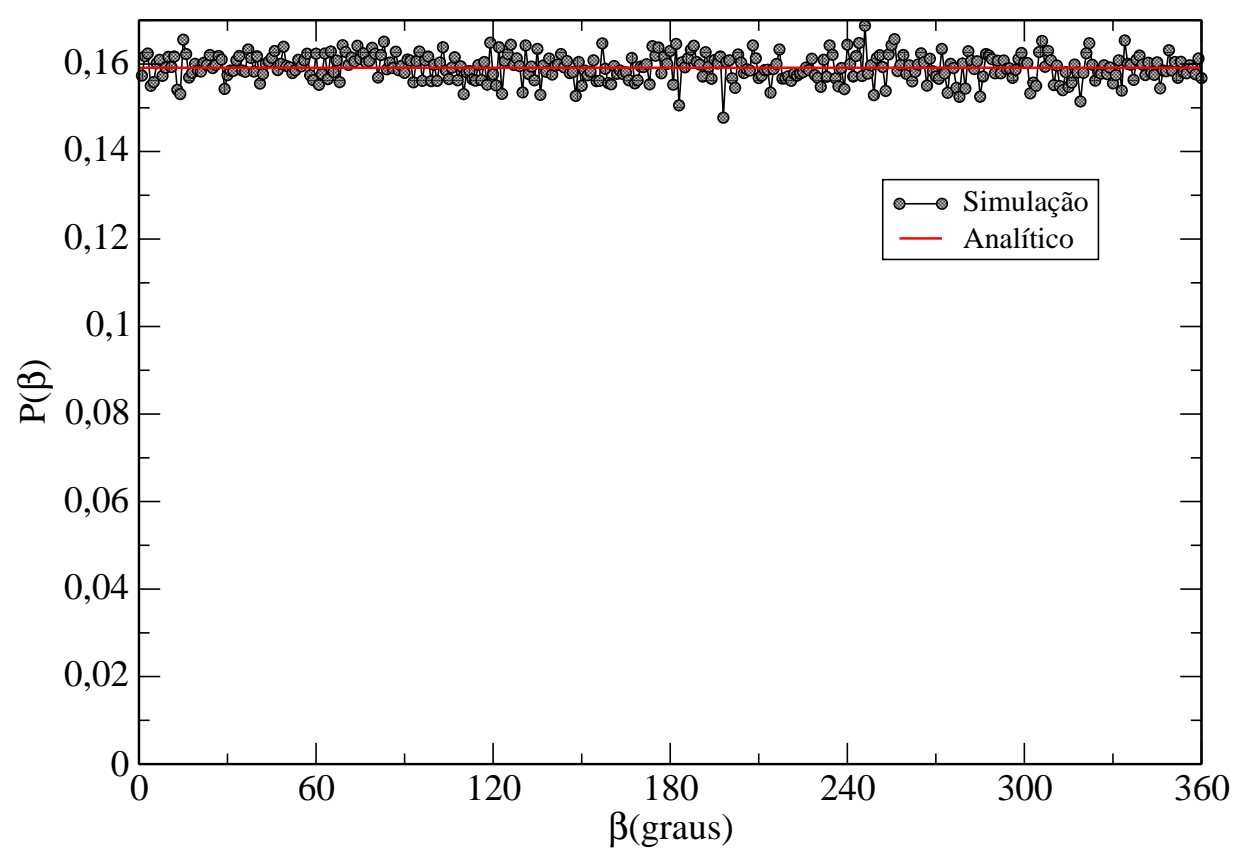

Figura 5.9: Distribuição do ângulo $\beta$

Se considerarmos $\left(n_{1}, n_{2}, n_{3}\right)$ as coordenadas cartesianas do vetor radial $\hat{n}$, então as com- 
ponentes do vetor unitário $\vec{N},\left(N_{1}, N_{2}, N_{3}\right)$ deve satisfazer as seguintes equações

$$
N_{1} n_{1}+N_{2} n_{2}+n_{3} N_{3}=0
$$

e

$$
N_{1}^{2}+N_{2}^{2}+N_{3}^{2}=1
$$

Esse vetor unitário $\vec{N}$ perpendicular ao vetor radial $\hat{n}$, servirá como eixo de rotação do vetor radial $\hat{n}$, que será rotacionado por um ângulo $\theta$, obter o vetor dipolo $\vec{\mu}$. A rotação foi feita usando a matriz de rotação tridimensional $D_{\hat{N}}(\theta)$, que tem sua dedução apresentada no apêndice $(\mathrm{C})$. Para usar a matriz de rotação tridimensional, é necessário ter um eixo de rotação, vetor a ser rotacionado e o valor do ângulo.

A seleção desse vetor unitário $\vec{N}$ usando as equações $(5.22)$ e (5.23) é feita levando em conta que estes vetores estão distribuídos aleatoriamente no plano perpendicular ao vetor radial $\hat{n}$. A escolha inicial das coordenadas do vetor unitário $\vec{N}$ foi feita com dependência das coordenadas do vetor radial $\hat{n}$. Se todas as componentes do vetor radial $\hat{n}$ forem não nulas, então o vetor normal escolhido foi $\left(-n_{2}, n_{1}, 0\right)$. Agora se alguma das componentes do vetor radial $\hat{n}$ for igual a zero, então a componente correspondente do vetor normal $\vec{N}$ será tomada como 1 e as outras demais como sendo zero. Reparem que as coordenadas do vetor $\vec{N}$ foram escolhidas à mão, mas desejamos esse vetor com direção aleatória. Para isso, escolhemos aleatoriamente um ângulo $\phi_{1}$ entre 0 e $2 \pi$, em seguida rotacionamos esse vetor normal $\vec{N}$ usando a matriz de rotação $D_{\hat{n}}\left(\phi_{1}\right)$ e como eixo de rotação o vetor radial $\hat{n}$ correspondente. Com isso obtivemos o vetor normal $\vec{N}$ com uma direção aleatória no plano perpendicular ao vetor radial $\hat{n}$. Tudo isso para que, quando rotacionarmos o vetor radial $\hat{n}$ por um ângulo $\theta$, usando como eixo de rotação o vetor normal $\vec{N}$, tenhamos o vetor dipolo $\vec{\mu}$ com orientação aleatória. Através deste procedimento, geramos as componentes do vetor $\vec{\mu}$ no referencial do laboratório, $\vec{\mu}^{L}=\left(\mu_{x}^{L}, \mu_{y}^{L}, \mu_{z}^{L}\right)$.

A escolha aleatória do ângulo $\theta$ que o vetor dipolo $\vec{\mu}$ irá fazer com o respectivo vetor radial $\hat{n}$, vai depender do modelo de rotação. 


\section{Rotação paralela à superfície da esfera}

No modelo de rotação no plano perpendicular ao vetor radial $\hat{n}$ da esfera e paralelo à superfície da esfera, o ângulo entre o vetor radial $\hat{n}$ e o vetor dipolo $\vec{\mu}$ é $90^{\circ}$. Se imaginarmos que cada vetor dipolo $\vec{\mu}$ tem como seu referencial o vetor radial $\hat{n}$ à superfície da esfera, como já temos um vetor unitário $\vec{N}$ perpendicular ao vetor radial $\hat{n}$, podemos adotar esse vetor $\vec{N}$ como sendo o próprio vetor dipolo $\vec{\mu}$.

Aplicamos o conceito de fotoseleção, com o intuito de observarmos quais dipolos dessa distribuição inicial serão considerados excitados. A probabilidade de um dipolo ser excitado é proporcional ao quadrado da componente $z$ do dipolo, $\left(\mu_{z}^{L}\right)^{2}$.

A seleção foi feita comparando o número aleatório com o quadrado da componente cartesiana $z$ do dipolo. Caso o número aleatório seja menor que o quadrado da componente $z$ do vetor dipolo $\vec{\mu}$, com relação ao referencial do laboratório, então o vetor dipolo $\vec{\mu}$ é aceito como excitado, caso contrário os passos iniciais são repetidos (escolha aleatória do vetor radial $\hat{n}$, escolha do vetor unitário $\vec{N}$ perpendicular ao vetor radial $\hat{n}$ e a fotoseleção). Esses processos foram feitos até obtermos $10^{6}$ dipolos excitados.

Este procedimento permite obter a distribuição inicial de dipolos na esfera no instante $t=0$ e a anisotropia de fluorescência $r_{0}$.

\section{Wobbling-cone}

No modelo de "Wobbling-cone"os dipolos têm seu movimento restrito a um cone. A restrição é dada pelo ângulo máximo entre o vetor radial $\hat{n}$ e o vetor dipolo $\vec{\mu}$.

Para sortearmos o ângulo entre o vetor radial $\hat{n}$ e o vetor dipolo $\vec{\mu}$, usamos o mesmo raciocínio usado no sorteio do ângulo $\alpha$, ângulo formado entre o vetor radial $\hat{n}$ e o eixo $z$ do laboratório. O número de vetores dipolo $\vec{\mu}$, que estão com orientação $\theta$ e $\theta+d \theta$ é proporcional a um elemento de superfície na esfera, $2 \pi \sin \theta d \theta$. A fração de vetores dipolos $\vec{\mu}$ com esta orientação é

$$
P(\theta) d \theta=\frac{2 \pi \sin \theta d \theta}{\int_{0}^{2 \pi} d \phi \int_{0}^{\theta_{\max }} \sin \theta d \theta}=\frac{\sin (\theta)}{1-\cos \theta_{\max }} d \theta
$$

portanto

$$
P(\theta)=\frac{\sin \theta}{1-\cos \theta_{\max }}
$$


onde $\theta_{\text {max }}$ é o ângulo máximo entre o vetor dipolo e o vetor radial. Usando a transformação apresentada na equação (5.8), chegamos na expressão que relaciona o número aleatório ao ângulo $\theta$, do movimento restrito no cone.

Para obtermos o vetor dipolo $\vec{\mu}$, rotacionamos o vetor radial $\hat{n}$ pelo ângulo $\theta$ sorteado, usando como eixo de rotação o vetor unitário $\vec{N}$ com direção aleatória, e o vetor oriundo dessa rotação é o vetor dipolo $\vec{\mu}$.

Aplicamos o conceito de fotoseleção para sabermos quais desses dipolos serão considerados excitados. A probabilidade de um dipolo ser excitado é proporcional a $\left(\mu_{z}^{L}\right)^{2}$. A seleção foi feita tomando um número aleatório e comparando com o quadrado da componente cartesiana $z$ do vetor dipolo $\vec{\mu}$, com relação à direção de polarização. Caso o número aleatório seja menor que o quadrado da componente $z$, então o vetor dipolo é aceito como excitado, caso contrário os passos iniciais são repetidos, escolha aleatória do vetor radial $\hat{n}$, escolha do vetor unitário $\vec{N}$ perpendicular ao vetor radial $\hat{n}$, escolha do vetor dipolo $\vec{\mu}$ e a fotoseleção. Esses processos foram feitos até obtermos $10^{6}$ dipolos excitados.

Com isso obtemos a distribuição inicial de dipolos na esfera no instante $t=0$ e a anisotropia de fluorescência $r_{0}$.

\subsubsection{Difusão}

Os vetores radiais $\hat{n}$, para os dipolos considerados excitados, são mantidos fixos e difundidos somente os vetores dipolos $\vec{\mu}$.

Cada um dos dois modelos tem seu processo de difusão, que leva em conta as características dos modelos.

\section{Rotação paralela à superfície da esfera}

Na difusão, o vetor dipolo $\vec{\mu}$, perpendicular ao vetor radial $\hat{n}$, rotaciona $1^{0}$ para a esquerda ou direita. Para isso escolhemos um número aleatório entre 0 e 1 , caso o número aleatório seja menor que 0,5 a rotação é para esquerda, caso contrário para a direita. Essa rotação é feita usando a matriz de rotação tridimensional $D_{\hat{n}}(\Delta \phi)$, usando como eixo de rotação o vetor radial $\hat{n}$. 
Em cada iteração, todas os vetores dipolos $\vec{\mu}$ são movimentados, e com a média da componente $z$ dos vetores dipolo, com relação à direção de polarização, é calculada a anisotropia, até que o sistema difunda completamente, isto é, até que seja atingido um regime estacionário da anisotropia.

\section{Wobbling-cone}

A difusão do vetor dipolo $\vec{\mu}$ em um cone é mais complexa, em comparação à rotação paralela à superfície da esfera. Uma iteração é composta de movimentos em $\theta$ e $\phi$.

Durante a relaxação do sistema, o ângulo entre o vetor dipolo $\vec{\mu}$ e o vetor radial $\hat{n}$, poderá aumentar, diminuir ou permanecer inalterado. A escolha é feita através da taxa de transição, apresentada na equação (5.14), e a probabilidade usada é a apresentada na equação (5.25). Sendo assim, escolhemos um número aleatório e caso ele seja maior que 0,5 o ângulo aumentará, caso contrário escolhemos um número aleatório e comparamos com a taxa de transição da equação (5.14), se o número aleatório for menor que a taxa de transição o ângulo diminuirá, caso contrário permanecerá com o mesmo valor.

Para que nessas escolhas não tivéssemos o ângulo $\theta>\theta_{\max }$ e $\theta<0$, impusemos condições de contorno nessas extremidades. Se em uma dessas escolhas, o deslocamento do vetor dipolo $\vec{\mu}$ ultrapassar $\theta_{\text {max }}$, a condição de contorno será $\theta_{N}=2 \cdot \theta_{\text {max }}-\theta_{v}$, onde $\theta_{v}$ é o ângulo maior que $\theta_{\max }$, para onde o vetor dipolo $\vec{\mu}$ deveria deslocar, e $\theta_{N}$ é o ângulo novo refletido na barreira. O tamanho do passo em $\theta$, corresponde a uma fração do comprimento de uma esfera de raio 1 , ou seja, $\Delta \theta=\frac{2 \pi}{n}$.

Caso o ângulo de deslocamento for menor que $\Delta \theta$, a condição de contorno será $\theta_{N}=$ $\Delta \theta-\theta_{v}$

A outra parte do deslocamento do vetor dipolo $\vec{\mu}$, é com relação ao ângulo $\phi$. Mas no deslocamento em $\phi$, deve ser levado em consideração o ângulo no qual o vetor dipolo $\vec{\mu}$ está. Isso porque, para cada valor de $\theta$, o deslocamento em $\phi$ será feito em uma circunferência de raio com dependência em $\theta, r=\sin \theta$. Para que o sistema tenha somente um único coeficiente de difusão, o tamanho do deslocamento linear na superfície da esfera deve ter o mesmo tamanho do deslocamento linear em $\theta$, então para que o deslocamento linear em $\phi$ tenha o mesmo tamanho que de $\theta$, devemos ter $\Delta \phi=\frac{2 \pi}{n \sin \theta}$. 
Sorteamos um número aleatório, caso seja menor que 0,5 , o deslocamento é no sentido horário, caso contrário, o deslocamento é no sentido anti-horário.

\subsubsection{Resultados}

\section{Rotação paralela à superfície da esfera}

A figura (5.10) mostra o resultado da simulação de Monte Carlo para a anisotropia de fluorescência da rotação do dipolo no plano perpendicular à normal da superfície da esfera. Os valores de $r(t)$ para $t=0$ e $t \rightarrow \infty$ concordam com os valores obtidos a partir da equação da difusão, $r(0)=0$ e $r(t \rightarrow \infty)=0,1$. Mas para podermos comparar os resultados da simulação com a expressão analítica, equação 4.34, é necessário obter o coeficiente de difusão da simulação. Fazendo uma expansão em Taylor da equação (4.34) para $t$ próximo de zero, temos

$$
r(t) \cong\left(\frac{3}{10}+\frac{1}{10}\right)-\left(4 \cdot \frac{3}{10}\right) D_{r} t
$$

que é a expressão de uma reta. O coeficiente angular dessa reta é proporcional ao coeficiente de difusão rotacional.

Usamos esse mesmo raciocínio para os dados da simulação, tomando apenas os primeiros dados e ajustando uma reta. O gráfico da figura (5.11) mostra esse ajuste. O coeficiente angular do ajuste foi dividido por $\left(4 \cdot \frac{3}{10}\right)$ conforme a equação $(5.26)$ e obtivemos o coeficiente de difusão $D_{r}=0,000153075$ passos $^{-1}$.

Com o coeficiente de difusão podemos colocar no mesmo gráfico o resultado analítico e o resultado simulado, para verificarmos se há concordância entre ambos resultados. O coeficiente de difusão é dado em unidade de passos $^{-1}$, então podemos multiplicar o eixo dos passos no gráfico da figura (5.10) pelo coeficiente obtido e termos o eixo em unidade adimensional. A figura (5.12) apresenta o confronto entre os dois resultados. Não é possível distinguir os dois resultados na figura.

\section{Wobbling-cone}

A figura 5.13 mostra os resultados das simulações de Monte Carlo para a anisotropia de fluorescência de uma distribuição de dipolos com rotação restrita a um cone. As simulações 


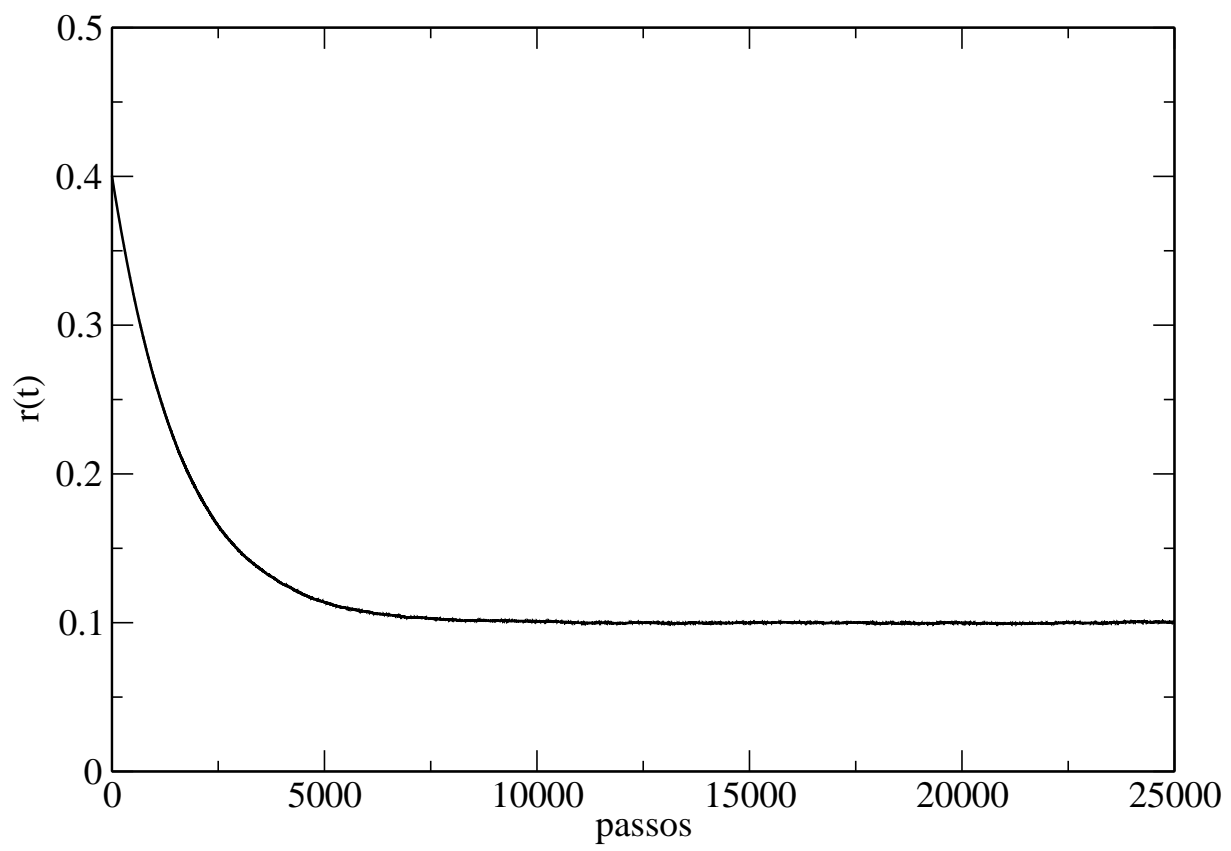

Figura 5.10: Resultdos da simulação da rotação no plano perpendicular à normal da superfície da esfera

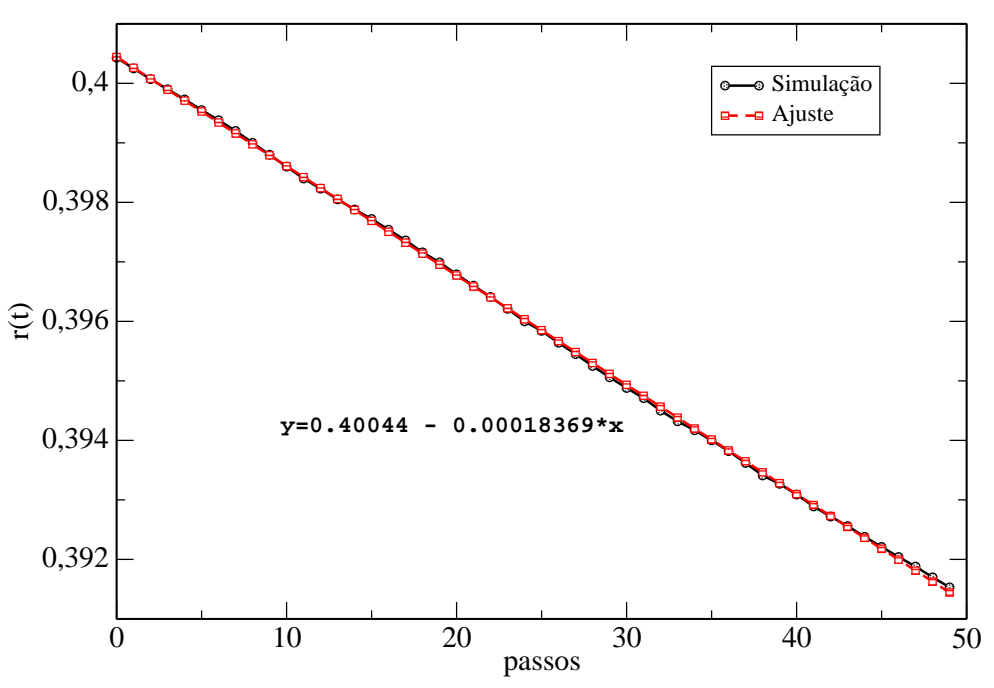

Figura 5.11: Ajuste para os dados iniciais da simulação da rotação do dipolo em um plano perpendicular à normal da superfície da esfera 


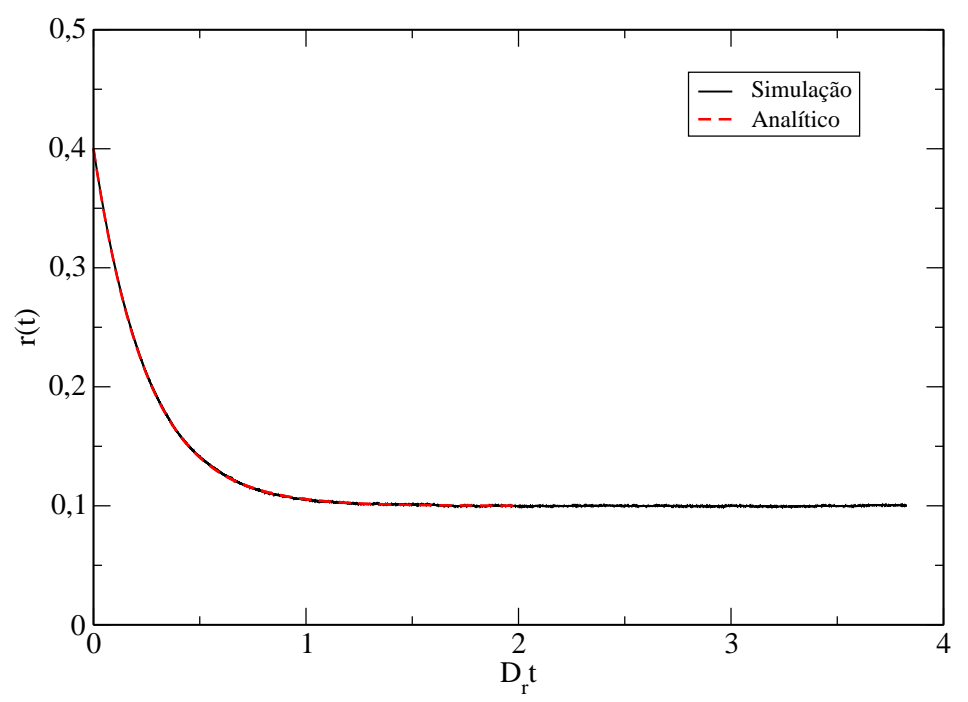

Figura 5.12: Comparação entre o resultado analítico e o resultado simulado para dipolos rotacionando no plano paralelo à superfície da esfera

foram feitas com diferentes tamanhos de passo e diferentes ângulos, $\theta_{\text {max }}$, de restrição de movimento.

As curvas da figura 5.13, para diferentes tamanhos de passos do deslocamento linear, concordam com os resultados analíticos, para $t=0$ e $t \rightarrow \infty$, para todos os ângulos $\theta_{\text {max }}$ do cone, obedecendo a relação 4.126 .

Para comparar os resultados ao longo de toda a relaxação, calculamos o coeficiente de difusão médio da simulação. Isso foi feito através do ajuste dos dados iniciais da simulação por uma reta. O coeficiente angular da reta, do ajuste, é proporcional ao coeficiente de difusão médio, sendo que, para a aproximação $t \approx 0$, a expressão da anisotropia para $o$ movimento restrito a um cone, é dada por

$$
r(t) \approx 0.4-6 \cdot 0.4\left\langle D_{r}\right\rangle t
$$

onde

$$
6\left\langle D_{r}\right\rangle=\sum_{m, n} A_{n}^{m} \sigma_{n}^{m} D_{r}
$$

Os gráficos das figuras 5.14 e 5.15, mostram os ajustes, para cada $\theta_{\text {max }}$ e os diferentes 


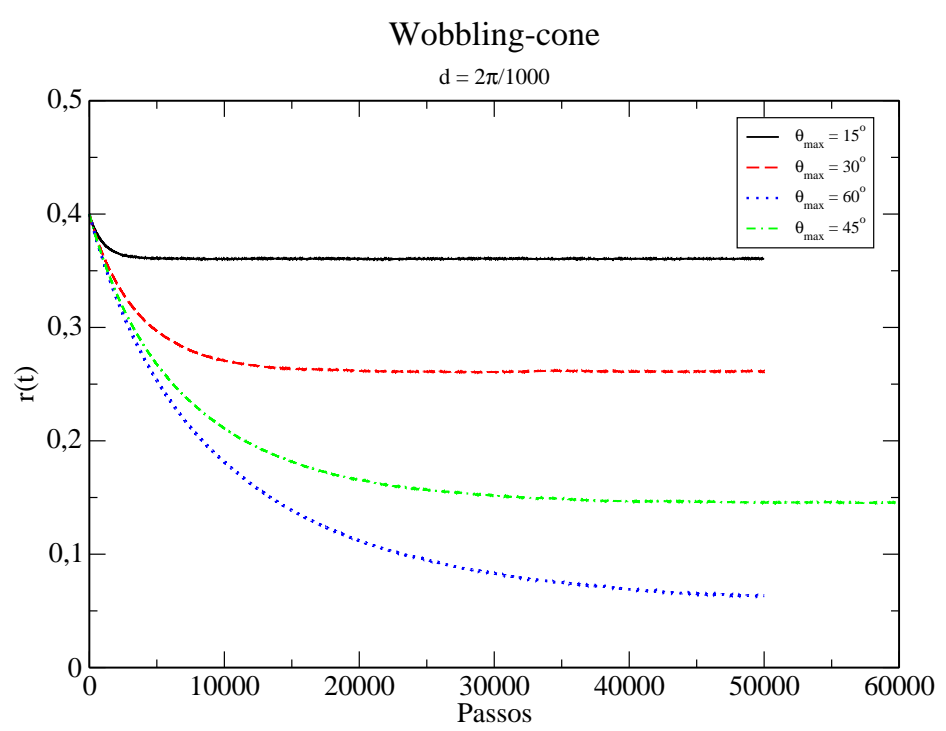

(a) Simulação de Monte Carlo para difusão restrita a um cone, com passos do tamanho $d=2 \pi / 1000$

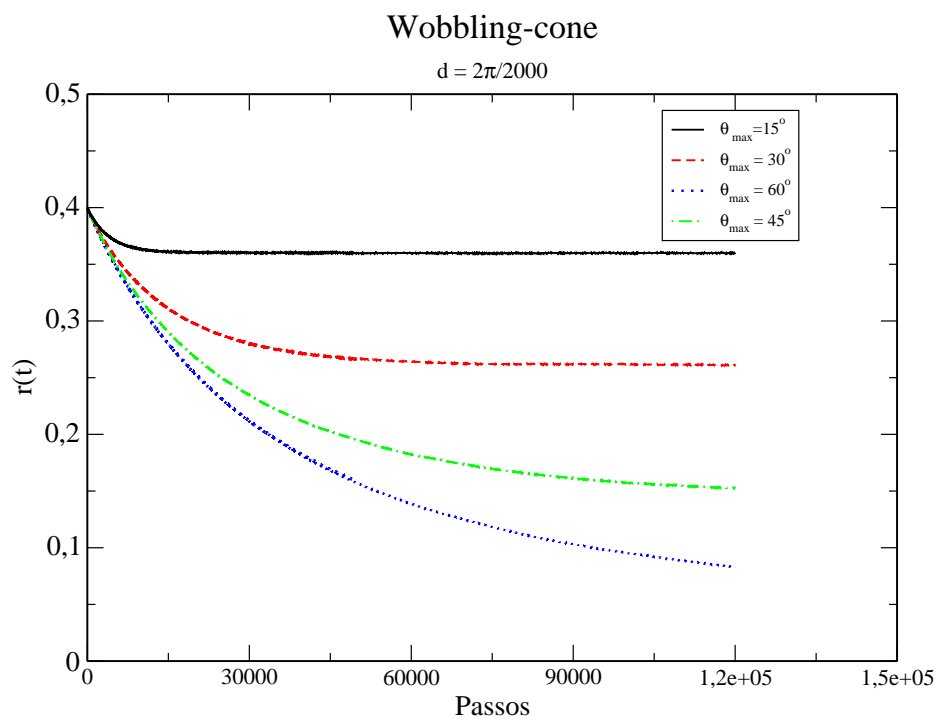

(b) Simulação de Monte Carlo para difusão restrita a um cone, com passos do tamanho $d=2 \pi / 2000$

Figura 5.13

tamanhos de deslocamento linear.

Obtidos os coeficientes de difusão, foi possível comparar os resultados das simulações, dos diferentes tamanhos de deslocamento linear, com o resultado analítico, para um dado $\theta_{\text {max }}$. Para que isso fosse possível, multiplicamos o eixo dos passos por $\left\langle D_{r}\right\rangle$, obtido dos ajustes, para que o eixo ficasse em unidade adimensional. A comparação é mostrada nos gráficos das figuras 5.16 e 5.17 , para os diferentes $\theta_{\max }$. As figuras 5.16 e 5.17 , mostram que 


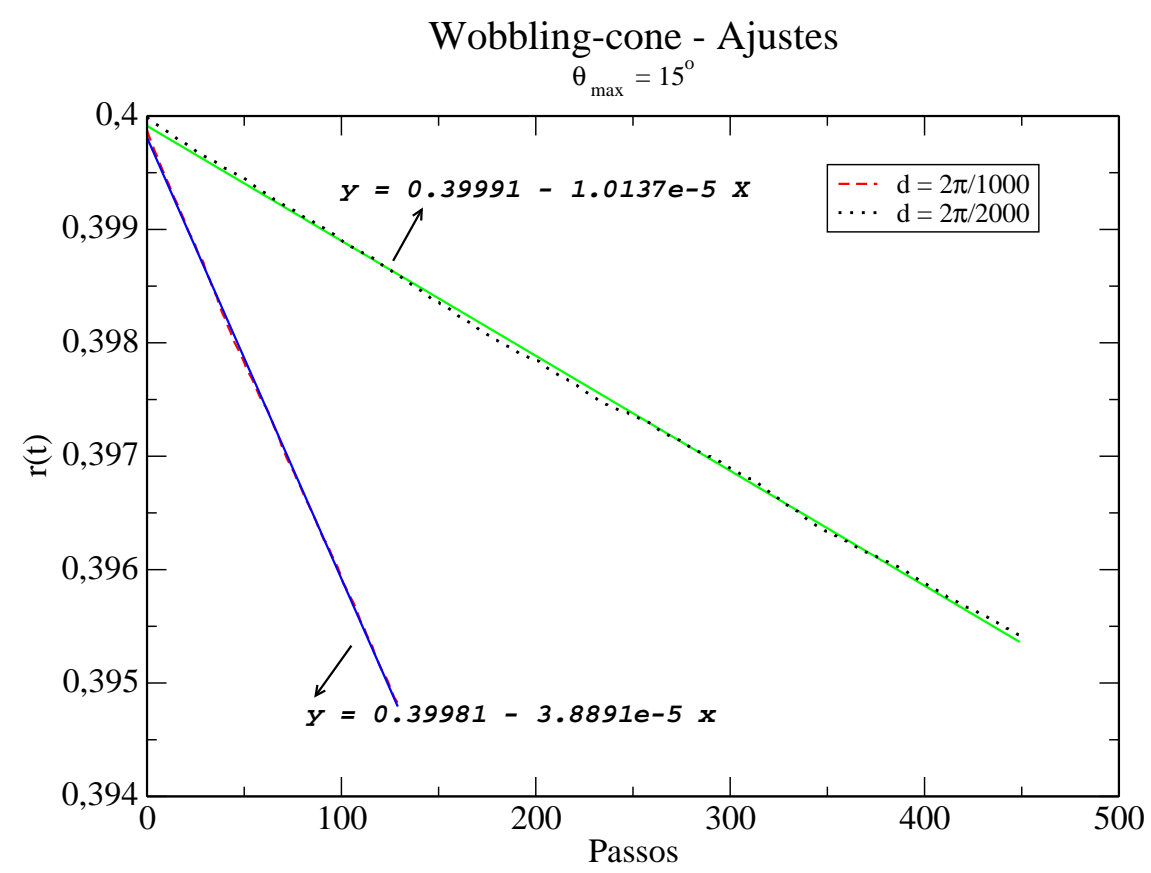

(a)

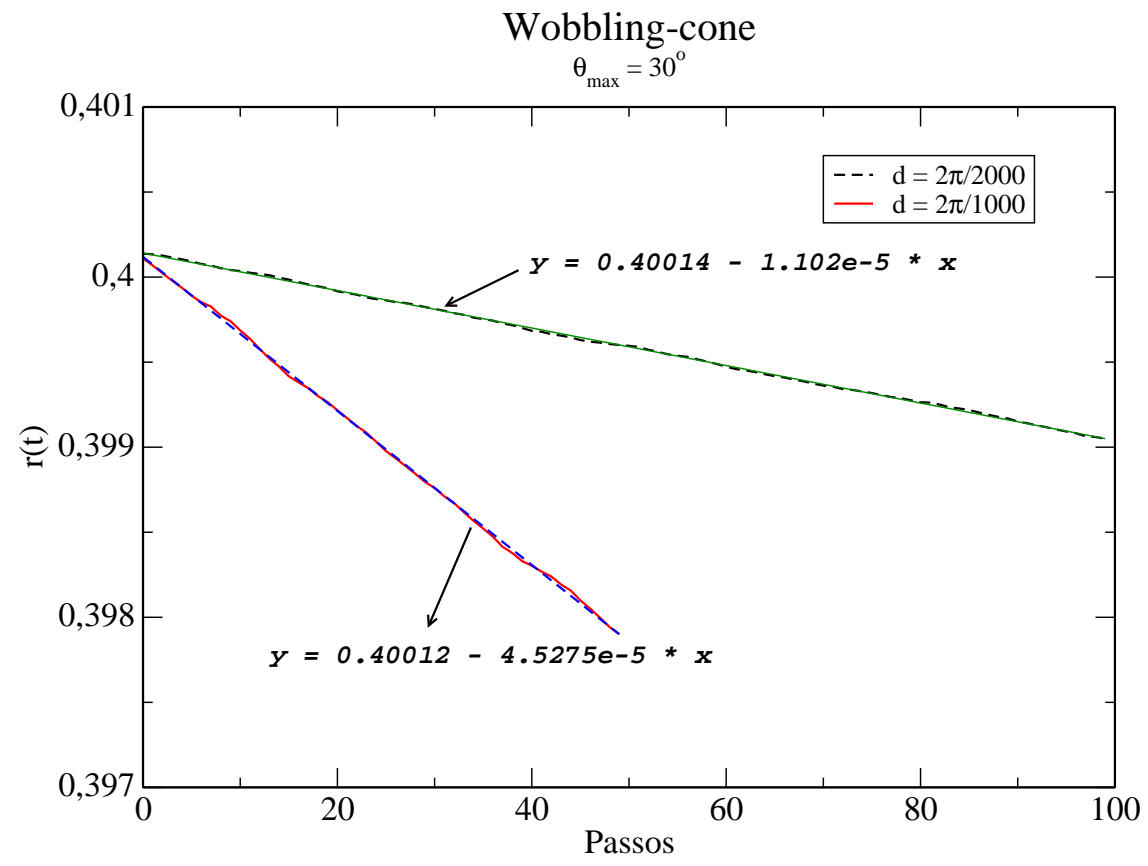

(b)

Figura 5.14: Ajuste de reta dos dados iniciais da simulação de Monte Carlo, para os dois tamanhos de deslocamento linear, em um mesmo $\theta_{\max }$

as simulações concordam com a previsão analítica, exceto no caso $\theta_{\max }=15^{0}$, para o qual há um pequena discrepância. Mas devemos levar em conta que a concordância da simulação com o valor analítico vai depender da qualidade com que é feito o ajuste de reta para os 


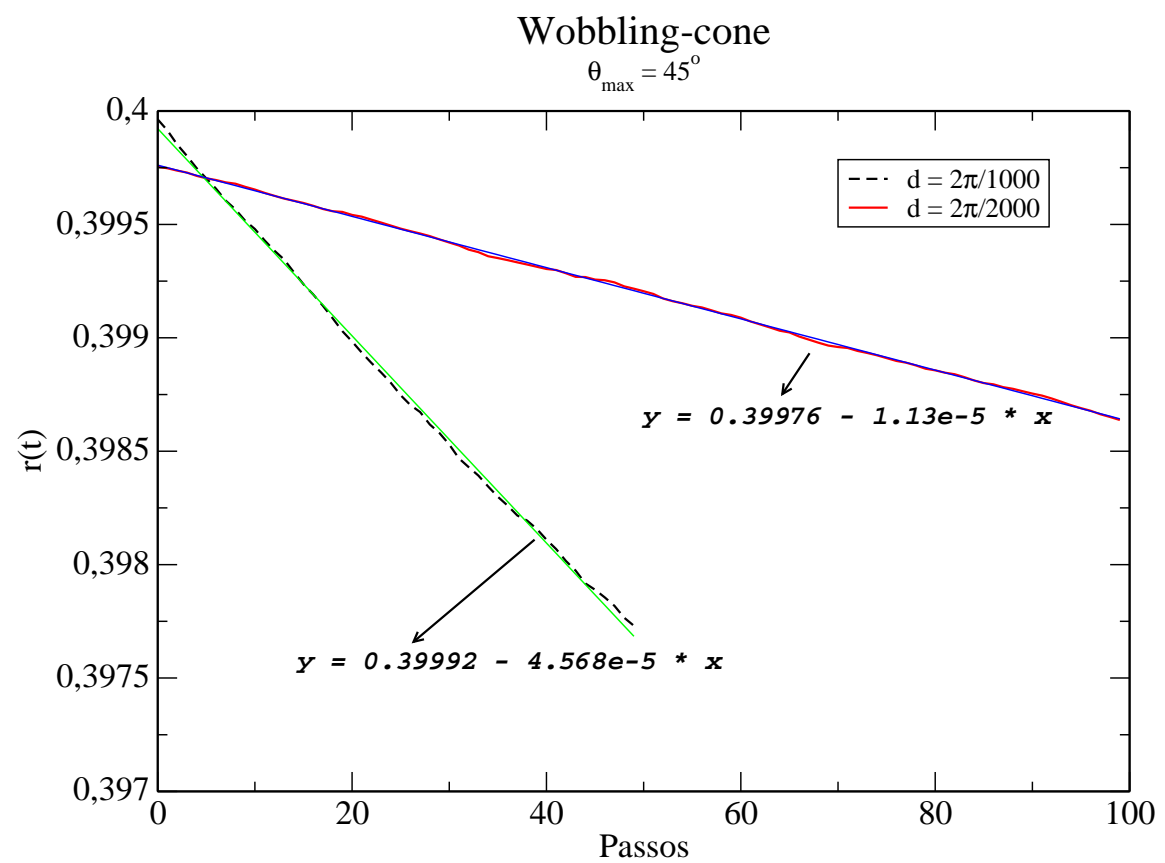

(a)

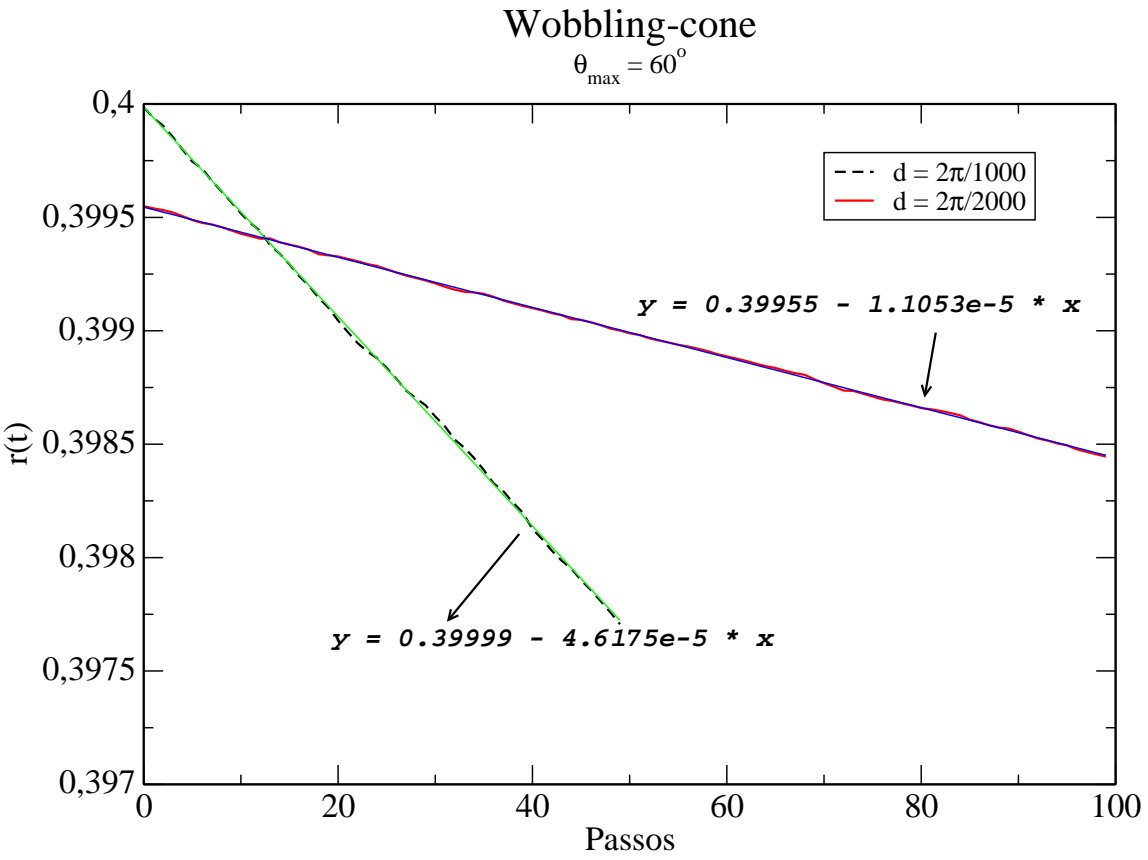

(b)

Figura 5.15: Ajuste de reta dos dados iniciais da simulação de Monte Carlo, para os dois tamanhos de deslocamento linear, em um mesmo $\theta_{\max }$

pontos iniciais.

Para fazer um estudo exploratório de interpretação dos dados experimentais para o DPH, figura 5.18, usamos como base os resultados obtidos nos modelos de rotação restrita. Supondo 


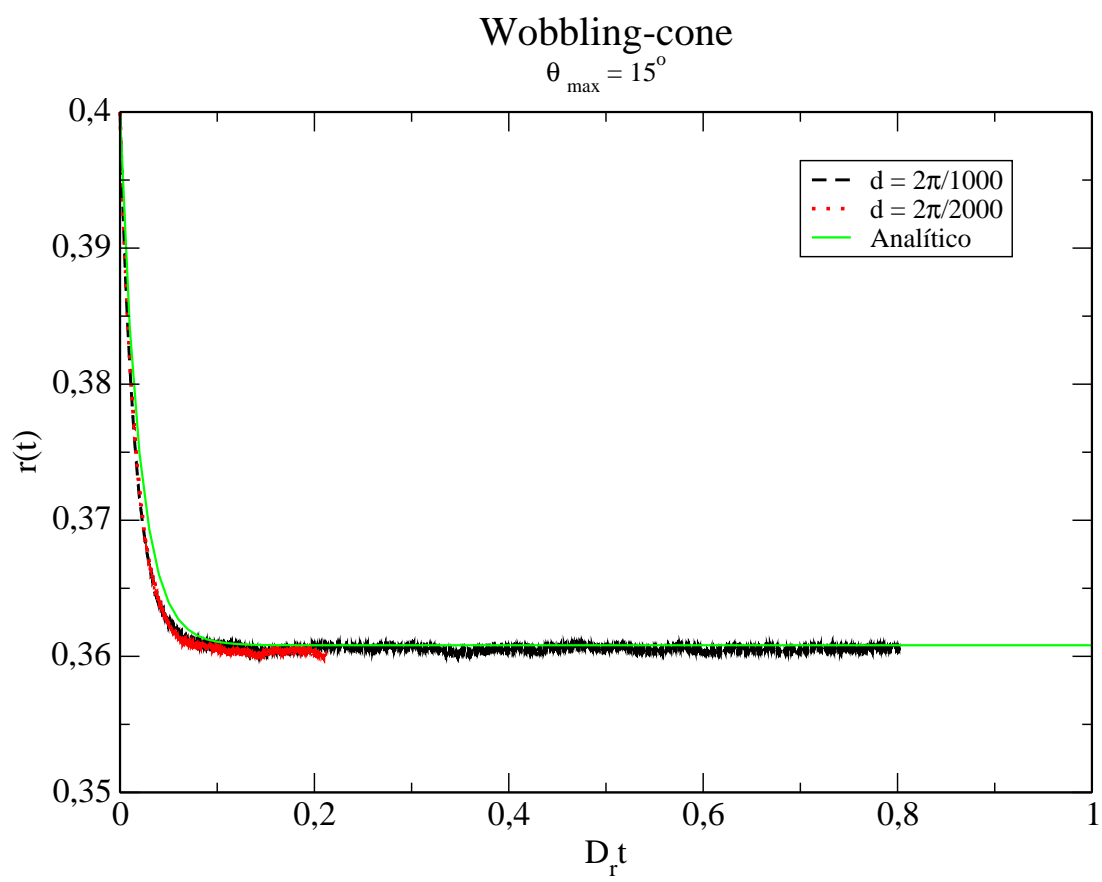

(a)

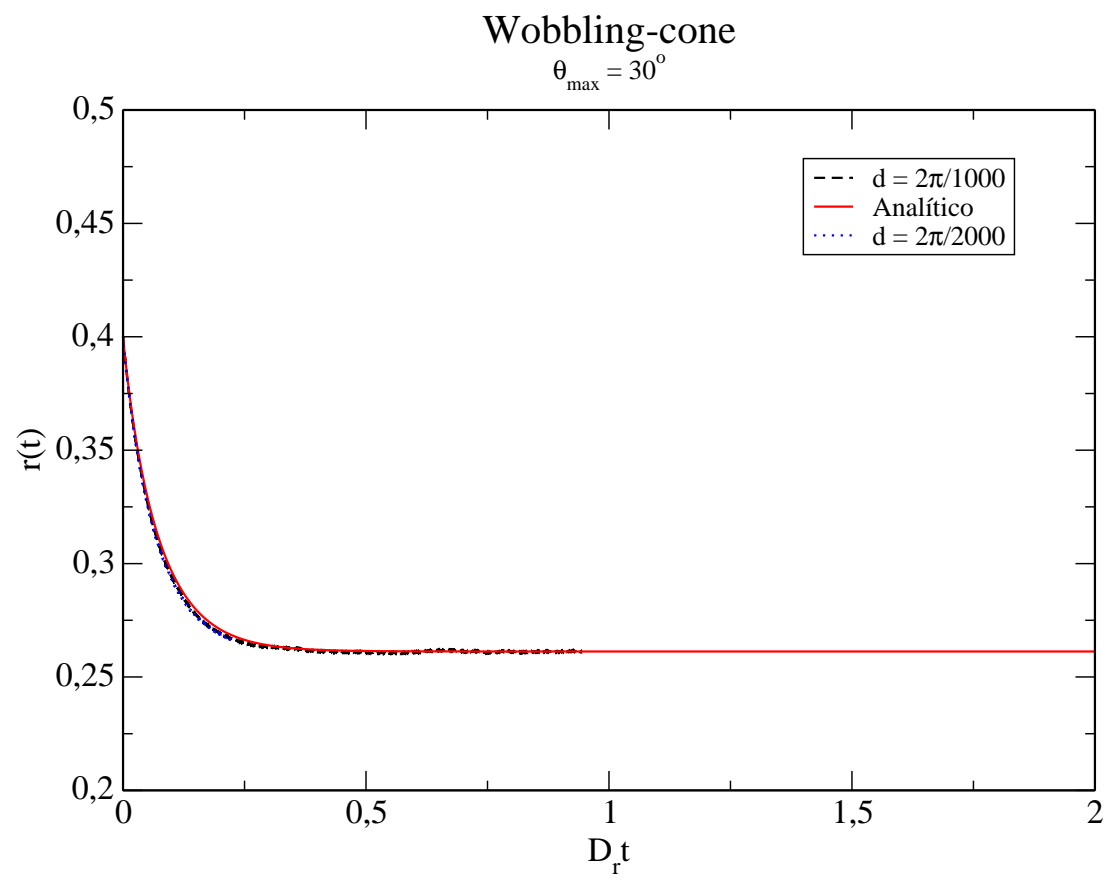

(b)

Figura 5.16: Comparação dos dados da simulação de Monte Carlo, para dois tamanhos de deslocamento linear, com o valor analítico

a existência de duas populações da molécula de DPH incorporada em membranas de DPPC, como proposto em [15, 16], somamos a anisotropia de fluorescência da rotação restrita em um cone, com $\theta_{\max }=30^{\circ}$, com a anisotropia de fluorescência da rotação paralela à superfície da 


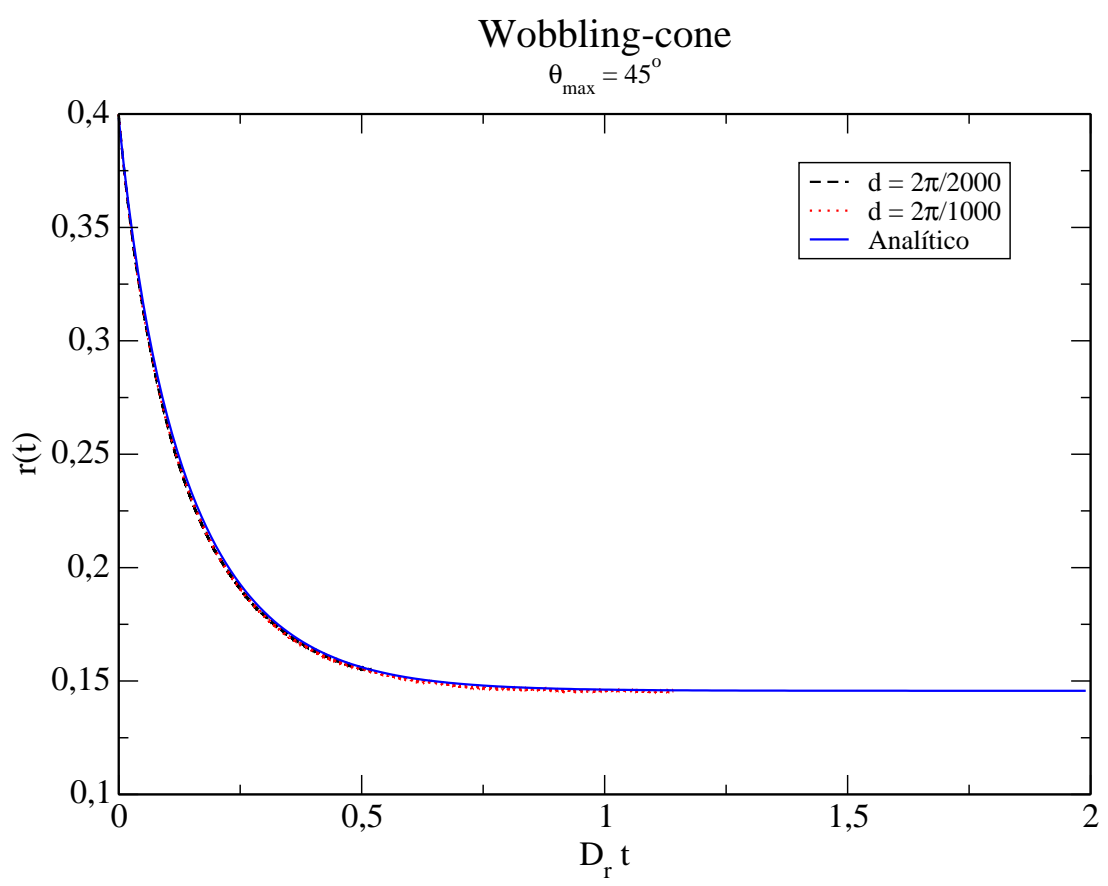

(a)

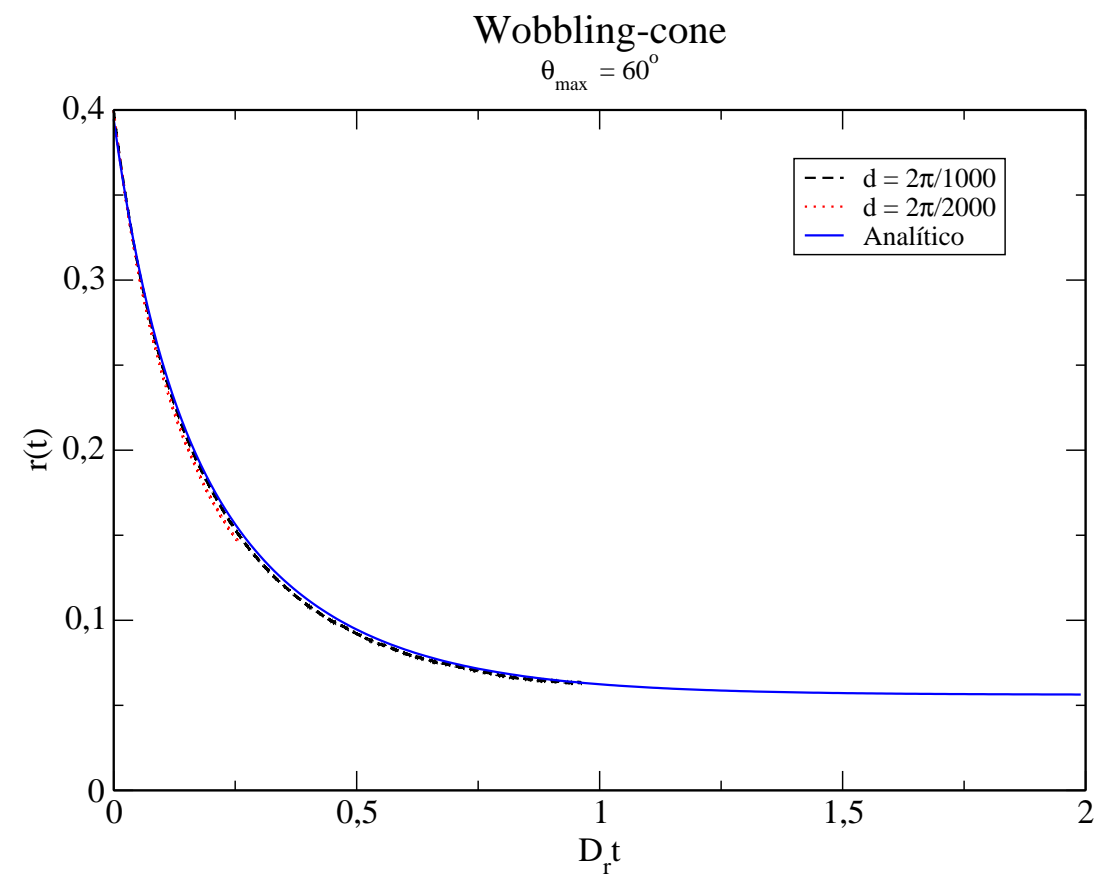

(b)

Figura 5.17: Comparação dos dados da simulação de Monte Carlo, para dois tamanhos de deslocamento linear, com o valor analítico

esfera, em diferentes proporções. Na figura 5.19, apresentamos os resultados dessa operação.

Para exemplificar a utilidade do estudo efetuado nesta dissertação, para a interpretação 


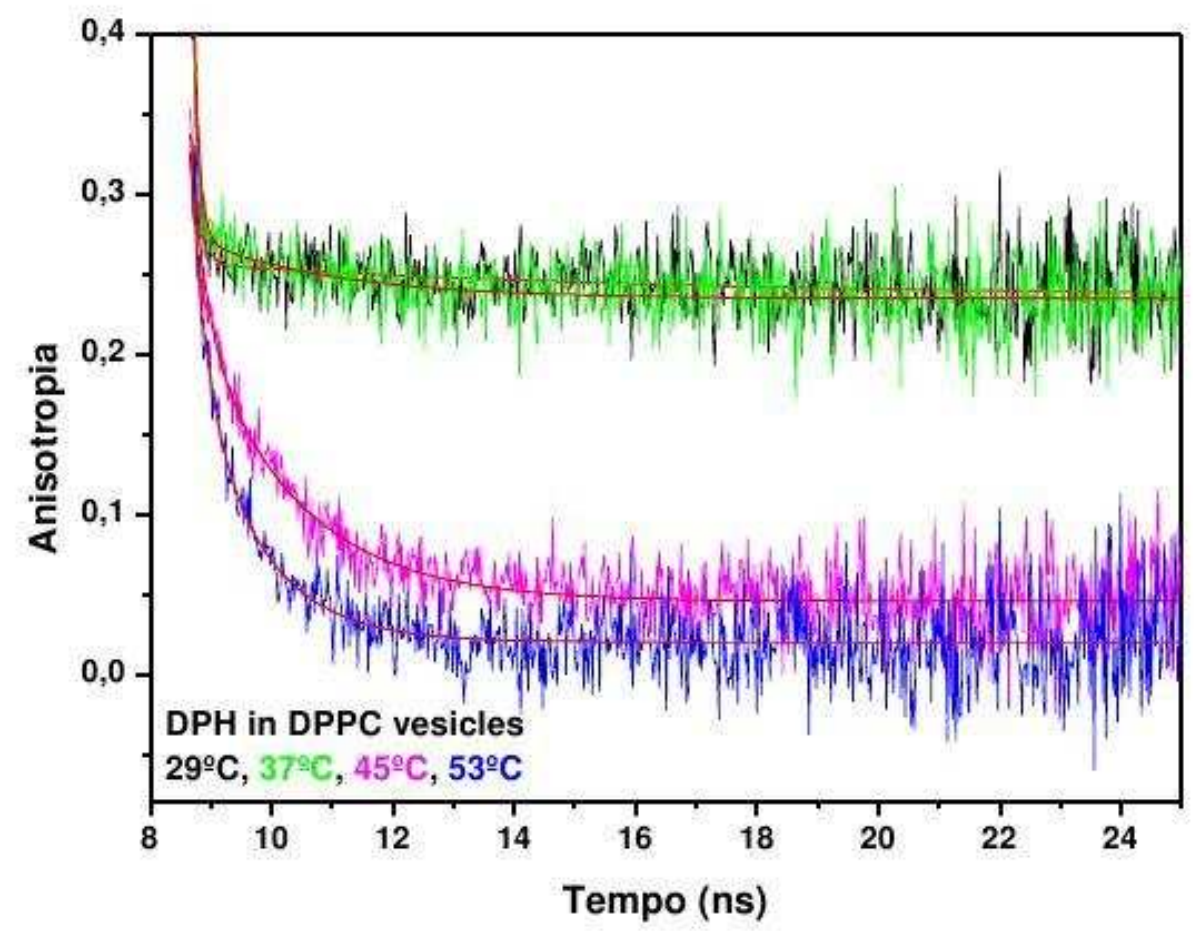

Figura 5.18: Dados experimentais da sonda DPH incorporada em membrana de DPPC

dos dados experimentais, comparamos as curvas experimentais para as temperaturas menores, com nossas curvas teóricas de composição de rotação no cone e rotação no plano paralelo à superfície. O resultado experimental para a anisotropia de fluorescência para tempos longos é $r(\infty) \approx 0,25$, para $29^{\circ} \mathrm{C}$ e $37^{\circ} \mathrm{C}$. Examinando a figura 5.19, verificamos que esse valor corresponde a aproximadamente $10 \%$ de dipolos rotacionando no plano paralelo à superfície da esfera e $90 \%$ dos dipolos em movimento dentro do cone de $\theta_{\max }=30^{\circ}$.

Podemos escrever uma relação entre o valor limite experimental $r_{\text {exp }}(\infty)$ e os valores limite teóricos das duas populações, $r_{\text {cone }}\left(\theta_{\max }, \infty\right)$ e $r_{\text {plano }}(\infty)=0.1$, na forma

$$
r_{\text {exp }}(\infty)=x \cdot r_{\text {cone }}\left(\theta_{\max }, \infty\right)+(1-x) \cdot 0,1
$$

onde $x$ é a fração de dipolos em cone e $(1-x)$ é a fração de dipolos nos planos intercamadas. Embora a equação não defina completamente os valores de $\theta_{\max }$ e de $x$, ela restringe possíveis valores para a fração de cada população e de valores $\theta_{\max }$ para os dipolos em cone. 

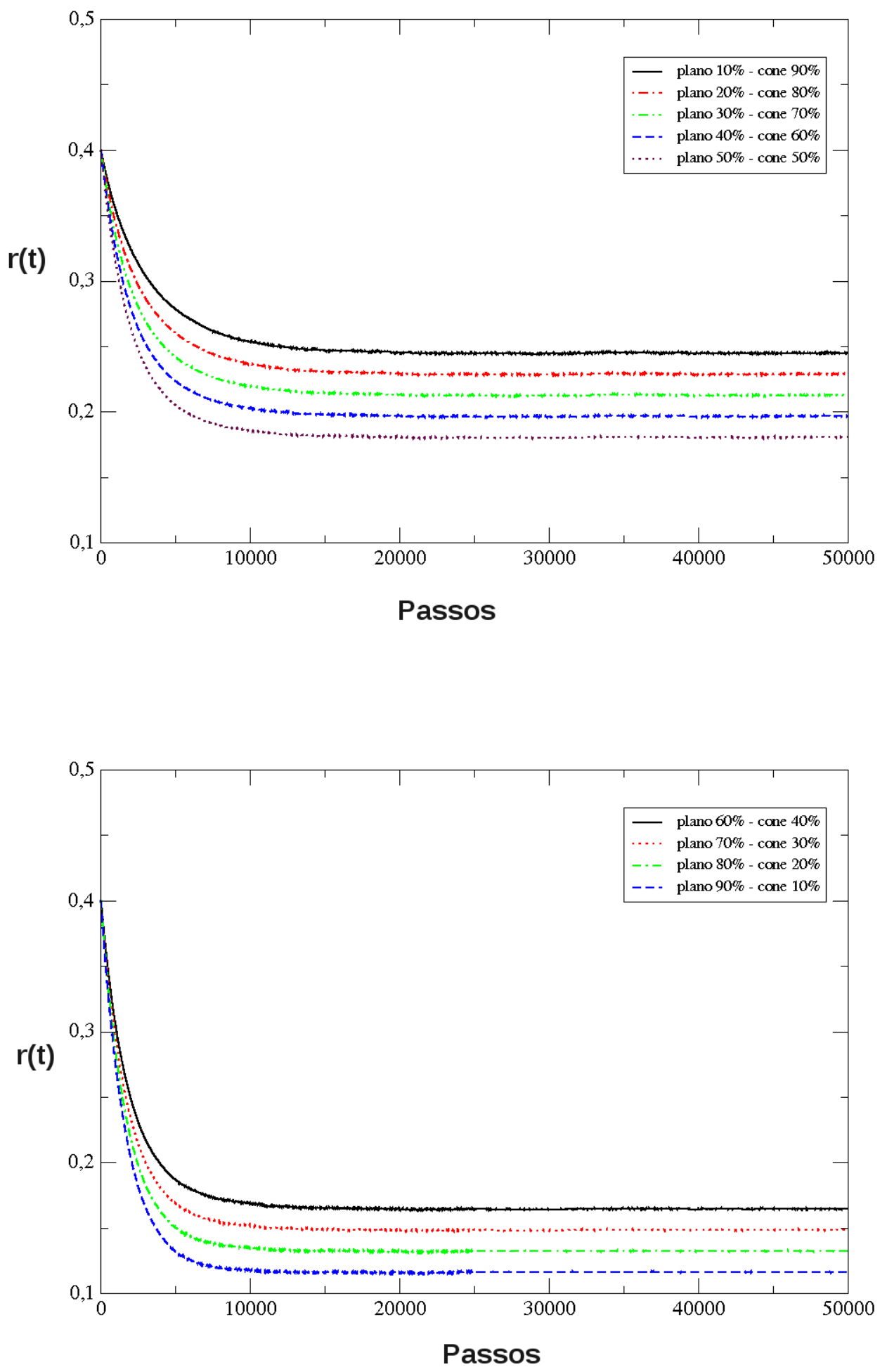

Figura 5.19: Combinação de fraçôes de sondas rotacionando no plano paralelo à superfície da membrana e rotacionando no cone 
78 DIFUSÃO ROTACIONAL E ANISOTROPIA DE FLUORESCENNCIA - SIMULAÇÕES NUMÉRICAS 


\section{Capítulo 6}

\section{Conclusão}

Neste trabalho, desenvolvemos uma metodologia para a interpretação de dados experimentais de difusão rotacional de moléculas fluorescentes em meios anisotrópicos.

Nosso estudo baseou-se em dois modelos de rotação restrita na geometria esférica, propostos em trabalhos anteriores para fluoróforos uniaxiais incorporados em membranas. Fizemos um estudo sistemático da anisotropia de fluorescência exibida por estes dois modelos, através de uma abordagem analítica, da resolução da equação de difusão, e através de simulações numéricas de passeio aleatório rotacional, baseadas no método de Monte Carlo. A comparação dos resultados obtidos através dos dois métodos permitiu estabelecer confiabilidade na abordagem de simulações numéricas, que inclui a escolha correta de parâmetros da simulação, tanto para a difusão rotacional de um dipolo, como para a distribuição na geometria do modelo escolhido do conjunto de dipolos.

A técnica desenvolvida pode facilmente ser estendida a modelos que incluam outras restrições, à composição de populações com os dois tipos de movimento, ou, ainda a movimentos compostos do mesmo fluoróforo. Como aplicação inicial dos resultados obtidos, demonstramos que propostas existentes na literatura a respeito da distribuição do fluróforo DPH em duas populações na bicamada lipídica possui restrições importantes. Essas restrições, facilmente calculadas para o regime de relaxação da anisotropia da fluorescência, inviabilizam hipóteses que atribuem à população paralela ao plano da membrana ordenada porcentagens altas, de 80 a $90 \%$, pois permitem um máximo de $10 \%$ a essa população. 


\section{Apêndice A}

\section{Parâmetros}

\section{A.1 Rotação livre}

\section{A.1.1 Ângulo polar médio}

Estamos considerando o ângulo polar como sendo o ângulo entre a direção de polarização, eixo $z$, e o vetor dipolo.

Conhecida a probabilidade obtida no capítulo (4)

$$
f(\theta, t)=P_{2}(\cos \theta) \exp \left(-6 D_{r} t\right)+\frac{1}{2}
$$

onde

$$
P_{2}(\cos \theta)=\frac{3}{2} \cos ^{2} \theta-\frac{1}{2}
$$

Desejamos calcular

$$
\langle\theta\rangle=\int_{0}^{\pi} \theta f(\theta, t) \sin \theta d \theta
$$

com isso temos

$$
\begin{array}{r}
\langle\theta\rangle=\int_{0}^{\pi} \theta\left[\left(\frac{3}{2} \cos ^{2} \theta \exp \left(-6 D_{r} t\right)-\frac{1}{2}\right)+\frac{1}{2}\right] \sin \theta d \theta= \\
=\frac{3}{2} \exp \left(-6 D_{r} t\right) \underbrace{\int_{0}^{\pi} \theta \cos ^{2} \theta \sin \theta d \theta}_{I_{1}}+\frac{1}{2}\left[1-\exp \left(-6 D_{r} t\right)\right] \underbrace{\int_{0}^{\pi} \theta \sin \theta d \theta}_{I_{2}}
\end{array}
$$


Resolvendo cada uma das integrais separadamente, começando por $I_{1}$

$$
I_{1}=\int_{0}^{\pi} \theta \cos ^{2} \theta \sin \theta d \theta=-\int_{0}^{\pi} \theta \frac{d\left(\cos ^{3} \theta\right)}{3}=\frac{\pi}{3}+\frac{1}{3} \int_{0}^{\pi} \cos ^{3} \theta d \theta=\frac{\pi}{3} .
$$

Resolvendo $I_{2}$

$$
I_{2}=\int_{0}^{\pi} \theta \sin \theta d \theta=\pi
$$

Substituindo as equações (A.6) e (A.7) na equação (A.3) temos

$$
\langle\theta\rangle=\frac{\pi}{2}
$$

\section{A.1.2 Ângulo polar médio quadrático}

Desejamos obter

$$
\begin{aligned}
\left\langle\theta^{2}\right\rangle & =\int_{0}^{\pi} \theta^{2} f(\theta, t) \sin \theta d \theta \\
& =\frac{3}{2} \exp \left(-6 D_{r} t\right) \underbrace{\int_{0}^{\pi} \theta^{2} \cos ^{2} \theta \sin \theta d \theta}_{I_{1}}+\frac{1}{2}\left(1-\exp \left(-6 D_{r} t\right)\right) \underbrace{\int_{0}^{\pi} \theta^{2} \sin \theta d \theta}_{I_{2}}
\end{aligned}
$$

Resolvendo $I_{1}$ temos

$$
I_{1}=\int_{o}^{\pi} \theta^{2} \cos ^{2} \theta \sin \theta d \theta=\frac{\pi^{2}}{3}-\frac{28}{27}
$$

Agora resolvendo $I_{2}$ temos

$$
I_{2}=\int_{0}^{\pi} \theta^{2} \sin \theta d \theta=\pi^{2}-4
$$

Usando os resultados obtidos nas equações (A.11) e (A.12) na equação (A.10) temos

$$
\left\langle\theta^{2}\right\rangle=\frac{4}{9} \exp \left(-6 D_{r} t\right)+\left(\frac{\pi^{2}}{2}-2\right)
$$




\section{A.1.3 Dispersão}

A dispersão é dada por

$$
\sigma^{2}=\left\langle\theta^{2}\right\rangle-\langle\theta\rangle^{2}
$$

Substituindo as equações (A.8) e (A.13) em (A.14), temos

$$
\sigma^{2}=\frac{4}{9} \exp \left(-6 D_{r} t\right)+\frac{\pi^{2}}{4}-2
$$


84 APÊNDICE A 


\section{Apêndice B}

\section{Coeficientes simulação numérica}

\begin{tabular}{|c|c|c|c|c|c|c|}
\hline \multicolumn{7}{|c|}{ Coeficientes $A_{n}^{m}$} \\
\hline & $\mathrm{m}=0$ & $\mathrm{~m}=1$ & $\mathrm{~m}=2$ & $\mathrm{~m}=0$ & $\mathrm{~m}=1$ & $\mathrm{~m}=2$ \\
\hline $\mathrm{n}=1$ & 0,989282 & 0,011219 & 0,000014 & 0,955774 & 0,043979 & 0,000221 \\
\hline $\mathrm{n}=2$ & 0,000006 & 0,000116 & 0,000000 & 0,000137 & 0,000426 & 0,000006 \\
\hline $\mathrm{n}=3$ & 0,000000 & 0,000018 & 0,000000 & 0,000009 & 0,000065 & 0,000001 \\
\hline $\mathrm{n}=4$ & 0,000000 & 0,000005 & 0,000000 & 0,000001 & 0,000019 & 0,000000 \\
\hline & $\theta_{\max }=15^{\circ}$ & & & $\theta_{\text {max }}=20^{\circ}$ \\
\hline $\mathrm{n}$ & $\mathrm{m}=0$ & $\mathrm{~m}=1$ & $\mathrm{~m}=2$ & $\mathrm{~m}=0$ & $\mathrm{~m}=1$ & $\mathrm{~m}=2$ \\
\hline $\mathrm{n}=1$ & 0,902027 & 0,095669 & 0,001096 & 0,831016 & 0,162195 & 0,003370 \\
\hline $\mathrm{n}=2$ & 0,000720 & 0,000825 & 0,000028 & 0,002250 & 0,001169 & 0,000084 \\
\hline $\mathrm{n}=3$ & 0,000055 & 0,000125 & 0,000006 & 0,000176 & 0,000175 & 0,000016 \\
\hline $\mathrm{n}=4$ & 0,000010 & 0,000036 & 0,000002 & 0,000036 & 0,000051 & 0,000005 \\
\hline & $\theta_{\text {max }}=25^{\circ}$ & & \multicolumn{3}{c|}{$\theta_{\text {max }}=30^{\circ}$} \\
\hline $\mathrm{n}=2$ & 0,005397 & 0,001287 & 0,000188 & 0,010542 & 0,001186 & 0,000350 \\
\hline $\mathrm{n}=3$ & 0,000428 & 0,000183 & 0,000036 & 0,000775 & 0,000173 & 0,000067 \\
\hline $\mathrm{n}=4$ & 0,000094 & 0,000051 & 0,000012 & 0,000163 & 0,000051 & 0,000022 \\
\hline
\end{tabular}




\begin{tabular}{|c|c|c|c|c|c|c|}
\hline \multicolumn{4}{|c|}{$\theta_{\text {max }}=35^{\circ}$} & \multicolumn{3}{|c|}{$\theta_{\max }=40^{\circ}$} \\
\hline & $\mathrm{m}=0$ & $\mathrm{~m}=1$ & $\mathrm{~m}=2$ & $\mathrm{~m}=0$ & $\mathrm{~m}=1$ & $\mathrm{~m}=2$ \\
\hline $\mathrm{n}=1$ & 0,555146 & 0,395204 & 0,027737 & 0,457600 & 0,464956 & 0,044580 \\
\hline $\mathrm{n}=2$ & 0,018541 & 0,000759 & 0,000568 & 0,029371 & 0,000284 & 0,000827 \\
\hline $\mathrm{n}=3$ & 0,001301 & 0,000103 & 0,000107 & 0,001880 & 0,000042 & 0,000154 \\
\hline $\mathrm{n}=4$ & 0,000280 & 0,000028 & 0,000035 & 0,000393 & 0,000013 & 0,000050 \\
\hline \multicolumn{4}{|c|}{$\theta_{\max }=45^{\circ}$} & \multicolumn{3}{|c|}{$\theta_{\max }=50^{\circ}$} \\
\hline & $\mathrm{m}=0$ & $\mathrm{~m}=1$ & $\mathrm{~m}=2$ & $\mathrm{~m}=0$ & $\mathrm{~m}=1$ & $\mathrm{~m}=2$ \\
\hline $\mathrm{n}=1$ & 0,364237 & 0,521185 & 0,066732 & 0,278765 & 0,560066 & 0,094277 \\
\hline $\mathrm{n}=2$ & 0,043461 & 0,000000 & 0,001097 & 0,060611 & 0,000458 & 0,001338 \\
\hline $\mathrm{n}=3$ & 0,002521 & 0,000000 & 0,000202 & 0,003117 & 0,000056 & 0,000242 \\
\hline $\mathrm{n}=4$ & 0,000521 & 0,000000 & 0,000065 & 0,000635 & 0,000015 & 0,000078 \\
\hline \multicolumn{4}{|c|}{$\theta_{\max }=55^{\circ}$} & \multicolumn{3}{|c|}{$\theta_{\max }=60^{\circ}$} \\
\hline & $\mathrm{m}=0$ & $\mathrm{~m}=1$ & $\mathrm{~m}=2$ & $\mathrm{~m}=0$ & $\mathrm{~m}=1$ & $\mathrm{~m}=2$ \\
\hline $\mathrm{n}=1$ & 0,203656 & 0,580194 & 0,126896 & 0,140483 & 0,580608 & 0,163863 \\
\hline $\mathrm{n}=2$ & 0,080154 & 0,002284 & 0,001505 & 0,101386 & 0,006143 & 0,001556 \\
\hline $\mathrm{n}=3$ & 0,003533 & 0,000264 & 0,000268 & 0,003692 & 0,000654 & 0,000272 \\
\hline $\mathrm{n}=4$ & 0,000703 & 0,000069 & 0,000086 & 0,000718 & 0,000163 & 0,000086 \\
\hline \multicolumn{4}{|c|}{$\theta_{\max }=65^{\circ}$} & \multicolumn{3}{|c|}{$\theta_{\max }=70^{\circ}$} \\
\hline & $\mathrm{m}=0$ & $\mathrm{~m}=1$ & $\mathrm{~m}=2$ & $\mathrm{~m}=0$ & $\mathrm{~m}=1$ & $\mathrm{~m}=2$ \\
\hline $\mathrm{n}=1$ & 0,090231 & 0,561297 & 0,203859 & 0,052670 & 0,524669 & 0,246142 \\
\hline $\mathrm{n}=2$ & 0,123343 & 0,012946 & 0,001439 & 0,144697 & 0,023498 & 0,001230 \\
\hline $\mathrm{n}=3$ & 0,003520 & 0,001304 & 0,000240 & 0,002975 & 0,002232 & 0,000206 \\
\hline $\mathrm{n}=4$ & 0,000666 & 0,000324 & 0,000073 & 0,000541 & 0,000558 & 0,000065 \\
\hline
\end{tabular}




\begin{tabular}{|c|c|c|c|c|c|c|}
\hline \multicolumn{5}{|c|}{$\theta_{\text {max }}=75^{\circ}$} & \multicolumn{3}{c|}{$\theta_{\text {max }}=80^{\circ}$} \\
\hline & $\mathrm{m}=0$ & $\mathrm{~m}=1$ & $\mathrm{~m}=2$ & $\mathrm{~m}=0$ & $\mathrm{~m}=1$ & $\mathrm{~m}=2$ \\
\hline $\mathrm{n}=1$ & 0,026448 & 0,474336 & 0,288169 & 0,010327 & 0,413963 & 0,329301 \\
\hline $\mathrm{n}=2$ & 0,164323 & 0,038015 & 0,000859 & 0,180781 & 0,057232 & 0,000487 \\
\hline $\mathrm{n}=3$ & 0,002178 & 0,003292 & 0,000136 & 0,001222 & 0,004500 & 0,000079 \\
\hline $\mathrm{n}=4$ & 0,000387 & 0,000800 & 0,000041 & 0,000210 & 0,001075 & 0,000025 \\
\hline & $\theta_{\max }=85^{\circ}$ & & & $\theta_{\text {max }}=90^{\circ}$ \\
\hline & $\mathrm{m}=0$ & $\mathrm{~m}=1$ & $\mathrm{~m}=2$ & $\mathrm{~m}=0$ & $\mathrm{~m}=1$ & $\mathrm{~m}=2$ \\
\hline $\mathrm{n}=1$ & 0,002244 & 0,348233 & 0,366772 & 0,000000 & 0,281029 & 0,400295 \\
\hline $\mathrm{n}=2$ & 0,192880 & 0,081196 & 0,000141 & 0,200131 & 0,109300 & 0,000000 \\
\hline $\mathrm{n}=3$ & 0,000369 & 0,005708 & 0,000021 & 0,000000 & 0,006709 & 0,000000 \\
\hline $\mathrm{n}=4$ & 0,000059 & 0,001340 & 0,000006 & 0,000000 & 0,001537 & 0,000000 \\
\hline
\end{tabular}




\begin{tabular}{|c|c|c|c|c|c|c|}
\hline \multicolumn{7}{|c|}{ Coeficientes $l_{n}^{m}$} \\
\hline \multicolumn{4}{|c|}{$\theta_{\max }=5^{o}$} & \multicolumn{3}{|c|}{$\theta_{\max }=10^{\circ}$} \\
\hline & $\mathrm{m}=0$ & $m=1$ & $m=2$ & $\mathrm{~m}=0$ & $m=1$ & $\mathrm{~m}=2$ \\
\hline $\mathrm{n}=1$ & 0,0000 & 20,6155 & 34,5252 & 0,0000 & 10,0835 & 17,0520 \\
\hline $\mathrm{n}=2$ & 43,4110 & 60,5987 & 76,3571 & 21,4598 & 30,0567 & 37,9444 \\
\hline $\mathrm{n}=3$ & 79,8943 & 97,3220 & 113,7487 & 39,6995 & 48,4155 & 56,6349 \\
\hline $\mathrm{n}=4$ & 116,0804 & 133,6431 & 150,4266 & 57,7918 & 66,5748 & 74,9712 \\
\hline \multicolumn{4}{|c|}{$\theta_{\max }=15^{\circ}$} & \multicolumn{3}{|c|}{$\theta_{\max }=20^{\circ}$} \\
\hline & $\mathrm{m}=0$ & $\mathrm{~m}=1$ & $\mathrm{~m}=2$ & $\mathrm{~m}=0$ & $\mathrm{~m}=1$ & $\mathrm{~m}=2$ \\
\hline $\mathrm{n}=1$ & 0,0000 & 6,5842 & 11,2452 & 0,0000 & 4,8432 & 8,3553 \\
\hline $\mathrm{n}=2$ & 14,1446 & 19,8793 & 25,1472 & 10,4885 & 14,7931 & 18,7541 \\
\hline $\mathrm{n}=3$ & 26,3023 & 32,1154 & 37,6016 & 19,6044 & 23,9669 & 28,0886 \\
\hline $\mathrm{n}=4$ & 38,3630 & 44,2202 & 49,8230 & 28,6492 & 33,0440 & 37,2516 \\
\hline \multicolumn{4}{|c|}{$\theta_{\max }=25^{\circ}$} & \multicolumn{3}{|c|}{$\theta_{\max }=30^{\circ}$} \\
\hline & $\mathrm{m}=0$ & $\mathrm{~m}=1$ & $\mathrm{~m}=2$ & $\mathrm{~m}=0$ & $\mathrm{~m}=1$ & $\mathrm{~m}=2$ \\
\hline $\mathrm{n}=1$ & 0,0000 & 3,8056 & 6,6322 & 0,0000 & 3,1196 & 5,4928 \\
\hline $\mathrm{n}=2$ & 8,2960 & 11,7434 & 14,9226 & 6,8354 & 9,7121 & 12,3720 \\
\hline $\mathrm{n}=3$ & 15,5864 & 19,0790 & 22,3837 & 12,9083 & 15,8215 & 18,5830 \\
\hline $\mathrm{n}=4$ & 22,8213 & 26,3392 & 29,7110 & 18,9364 & 21,8702 & 24,6858 \\
\hline \multicolumn{4}{|c|}{$\theta_{\max }=35^{\circ}$} & \multicolumn{3}{|c|}{$\theta_{\max }=40^{\circ}$} \\
\hline & $\mathrm{m}=0$ & $\mathrm{~m}=1$ & $\mathrm{~m}=2$ & $\mathrm{~m}=0$ & $\mathrm{~m}=1$ & $\mathrm{~m}=2$ \\
\hline $\mathrm{n}=1$ & 0,0000 & 2,6347 & 4,6872 & 0,0000 & 2,2754 & 4,0905 \\
\hline $\mathrm{n}=2$ & 5,7930 & 8,2626 & 10,5536 & 5,0120 & 7,1770 & 9,1928 \\
\hline $\mathrm{n}=3$ & 10,9958 & 13,4957 & 15,8704 & 9,5619 & 11,7522 & 13,8381 \\
\hline $\mathrm{n}=4$ & 16,1619 & 18,6787 & 21,0980 & 14,0813 & 16,2857 & 18,4088 \\
\hline
\end{tabular}




\begin{tabular}{|c|c|c|c|c|c|c|}
\hline \multicolumn{4}{|c|}{$\theta_{\max }=45^{\circ}$} & \multicolumn{3}{|c|}{$\theta_{\max }=50^{\circ}$} \\
\hline & $\mathrm{m}=0$ & $\mathrm{~m}=1$ & $\mathrm{~m}=2$ & $\mathrm{~m}=0$ & $\mathrm{~m}=1$ & $\mathrm{~m}=2$ \\
\hline $\mathrm{n}=1$ & 0,0000 & 2,0000 & 3,6332 & 0,0000 & 1,7834 & 3,2740 \\
\hline $\mathrm{n}=2$ & 4,4053 & 6,3339 & 8,1373 & 3,9207 & 5,6607 & 7,2956 \\
\hline $\mathrm{n}=3$ & 8,4471 & 10,3970 & 12,2592 & 7,5556 & 9,3136 & 10,9979 \\
\hline $\mathrm{n}=4$ & 12,4633 & 14,4251 & 16,3186 & 11,1692 & 12,9371 & 14,6477 \\
\hline \multicolumn{4}{|c|}{$\theta_{\max }=55^{\circ}$} & \multicolumn{3}{|c|}{$\theta_{\max }=60^{\circ}$} \\
\hline & $\mathrm{m}=0$ & $m=1$ & $\mathrm{~m}=2$ & $\mathrm{~m}=0$ & $m=1$ & $\mathrm{~m}=2$ \\
\hline $\mathrm{n}=1$ & 0,0000 & 1,6096 & 2,9863 & 0,0000 & 1,4680 & 2,7526 \\
\hline $\mathrm{n}=2$ & 3,5249 & 5,1110 & 6,6095 & 3,1957 & 4,6542 & 6,0404 \\
\hline $\mathrm{n}=3$ & 6,8267 & 8,4279 & 9,9677 & 6,2195 & 7,6905 & 9,1109 \\
\hline $\mathrm{n}=4$ & 10,1107 & 11,7203 & 13,2820 & 9,2288 & 10,7067 & 12,1452 \\
\hline \multicolumn{4}{|c|}{$\theta_{\max }=65^{\circ}$} & \multicolumn{3}{|c|}{$\theta_{\max }=70^{\circ}$} \\
\hline & $\mathrm{m}=0$ & $\mathrm{~m}=1$ & $\mathrm{~m}=2$ & $\mathrm{~m}=0$ & $\mathrm{~m}=1$ & $\mathrm{~m}=2$ \\
\hline $\mathrm{n}=1$ & 0,0000 & 1,3513 & 2,5608 & 0,0000 & 1,2543 & 2,4025 \\
\hline $\mathrm{n}=2$ & 2,9178 & 4,2688 & 5,5614 & 2,6803 & 3,9396 & 5,1535 \\
\hline $\mathrm{n}=3$ & 5,7062 & 7,0674 & 8,3876 & 5,2665 & 6,5340 & 7,7694 \\
\hline $\mathrm{n}=4$ & 8,4829 & 9,8497 & 11,1846 & 7,8438 & 9,1156 & 10,3625 \\
\hline \multicolumn{4}{|c|}{$\theta_{\max }=75^{\circ}$} & \multicolumn{3}{|c|}{$\theta_{\max }=80^{\circ}$} \\
\hline & $\mathrm{m}=0$ & $\mathrm{~m}=1$ & $\mathrm{~m}=2$ & $\mathrm{~m}=0$ & $m=1$ & $\mathrm{~m}=2$ \\
\hline $\mathrm{n}=1$ & 0,0000 & 1,1731 & 2,2712 & 0,0000 & 1,1050 & 2,1626 \\
\hline $\mathrm{n}=2$ & 2,4751 & 3,6556 & 4,8026 & 2,2962 & 3,4083 & 4,4983 \\
\hline $\mathrm{n}=3$ & 4,8859 & 6,0724 & 7,2354 & 4,5532 & 5,6693 & 6,7700 \\
\hline $\mathrm{n}=4$ & 7,2902 & 8,4799 & 9,6513 & 6,8061 & 7,9243 & 9,0305 \\
\hline
\end{tabular}




\begin{tabular}{|c|c|c|c|c|c|c|}
\hline \multicolumn{3}{|c|}{$\theta_{\text {max }}=85^{\circ}$} & \multicolumn{3}{c|}{$\theta_{\max }=90^{\circ}$} \\
\hline & $\mathrm{m}=0$ & $\mathrm{~m}=1$ & $\mathrm{~m}=2$ & $\mathrm{~m}=0$ & $\mathrm{~m}=1$ & $\mathrm{~m}=2$ \\
\hline $\mathrm{n}=1$ & 0,0000 & 1,0478 & 2,0730 & 0,0000 & 1,0000 & 2,0000 \\
\hline $\mathrm{n}=2$ & 2,1390 & 3,1914 & 4,2328 & 2,0000 & 3,0000 & 4,0000 \\
\hline $\mathrm{n}=3$ & 4,2601 & 5,3145 & 6,3612 & 4,0000 & 5,0000 & 6,0000 \\
\hline $\mathrm{n}=4$ & 6,3792 & 7,4346 & 8,4841 & 6,0000 & 7,0000 & 8,0000 \\
\hline
\end{tabular}




\section{Apêndice C}

\section{Matriz de rotação}

Introduziremos vetores unitários $\vec{p}, \vec{q}$ ao longo dos eixos $x$ e $y$ respectivamente, figura C.1, onde os vetores $\vec{p}$ e $\vec{q}$ são ortogonais formando uma base $\{\vec{p}, \vec{q}\}$.

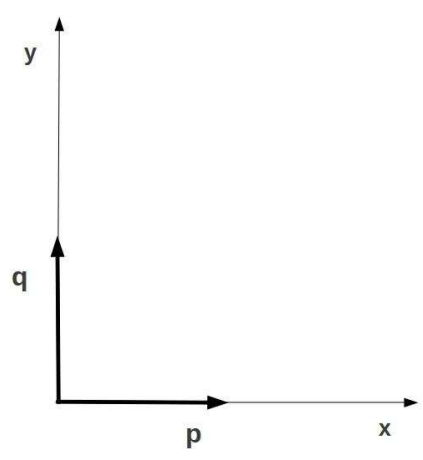

Figura C.1: Eixo de coordenadas com os vetores $\vec{p}$ e $\vec{q}$.

Faremos uma rotação dos eixos fixos na origem, por um ângulo $\theta$, mostrado na figura C.2.

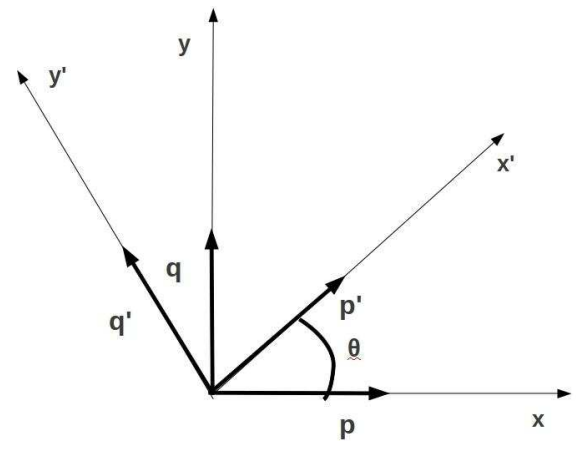

Figura C.2: Eixo de coordenadas rotacionado por um ângulo $\theta$. 
Com a rotação dos eixos passamos a ter uma nova base $\left\{\overrightarrow{p^{\prime}}, \overrightarrow{q^{\prime}}\right\}$ e as suas projeções ficam

$$
\begin{aligned}
\overrightarrow{p^{\prime}} & =\cos \theta \vec{p}+\sin \theta \vec{q} \\
\overrightarrow{q^{\prime}} & =-\sin \theta \vec{p}+\cos \theta \vec{q} .
\end{aligned}
$$

$\mathrm{Na}$ forma matricial a equação (C.2) fica

$$
\left(\begin{array}{c}
\overrightarrow{p^{\prime}} \\
\overrightarrow{q^{\prime}}
\end{array}\right)=\left(\begin{array}{rr}
\cos \theta & \sin \theta \\
-\sin \theta & \cos \theta
\end{array}\right)\left(\begin{array}{l}
\vec{p} \\
\vec{q}
\end{array}\right) .
$$

Agora faremos a mesma rotação do plano, apresentada anteriormente, porém em um espaço tridimensional. O raciocínio é o mesmo desenvolvido no espaço bidimensional, só que na origem teremos um eixo perpendicular ao plano no qual a rotação estará sendo feita em torno dele. A figura C.3 mostra essa rotação.

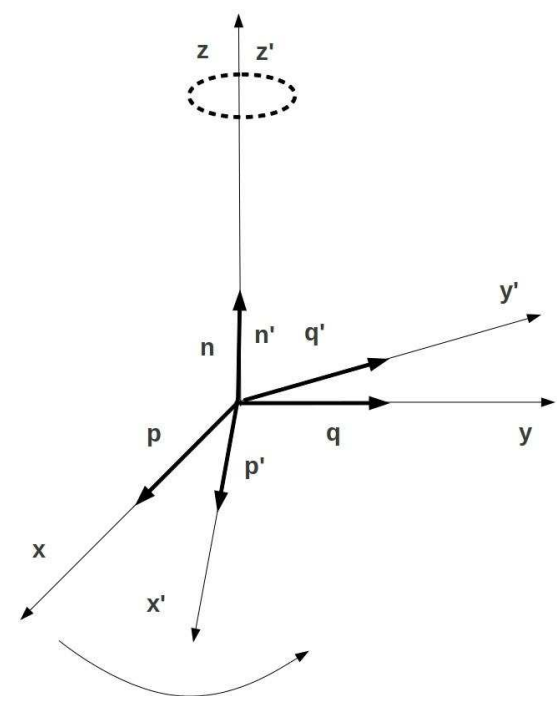

Figura C.3: Rotação do plano em torno do eixo perpendicular.

Como o eixo $z$ é fixo, ele tem a mesma forma nas duas bases. Assim temos

$$
\begin{aligned}
\overrightarrow{p^{\prime}} & =\cos \theta \vec{p}+\sin \theta \vec{q} \\
\overrightarrow{q^{\prime}} & =-\sin \theta \vec{p}+\cos \theta \vec{q} \\
\overrightarrow{n^{\prime}} & =\vec{n}
\end{aligned}
$$


ou ainda na forma matricial

$$
\left(\begin{array}{c}
\overrightarrow{p^{\prime}} \\
\overrightarrow{q^{\prime}} \\
\overrightarrow{n^{\prime}}
\end{array}\right)=\left(\begin{array}{rrr}
\cos \theta & \sin \theta & 0 \\
-\sin \theta & \cos \theta & 0 \\
0 & 0 & 1
\end{array}\right)\left(\begin{array}{c}
\vec{p} \\
\vec{q} \\
\vec{n}
\end{array}\right)
$$

Agora desejamos fazer a rotação de um vetor unitário no espaço tridimensional, juntamente com o plano, usando o eixo $z$ como eixo de rotação. A figura C.4 mostra a disposição do vetor no espaço.

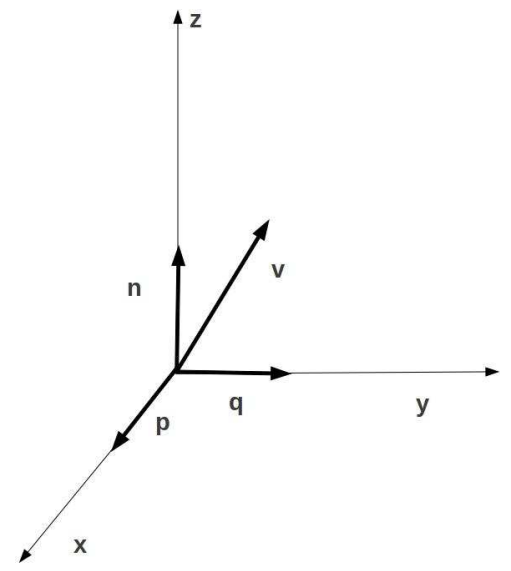

Figura C.4: Vetor unitário que será rotacionado no espaço

A projeção do vetor $\vec{v}$ nas direções $\vec{p}, \vec{q}$ e $\vec{n}$ é

$$
\vec{v}=(\vec{v} \cdot \vec{p}) \vec{p}+(\vec{v} \cdot \vec{q}) \vec{q}+(\vec{v} \cdot \vec{n}) \vec{n}
$$

Procuramos uma transformação

$$
\overrightarrow{v^{\prime}}=\mathbf{A} \vec{v}
$$

onde A é uma matriz de transformação.

A projeção de $\vec{v}$ é

$$
\vec{v}=\left(\vec{v} \cdot \overrightarrow{p^{\prime}}\right) \overrightarrow{p^{\prime}}+\left(\vec{v} \cdot \overrightarrow{q^{\prime}}\right) \overrightarrow{q^{\prime}}+\left(\vec{v} \cdot \overrightarrow{n^{\prime}}\right) \overrightarrow{n^{\prime}}
$$


e substituíndo a equação (C.6) na equação (C.10) temos

$$
\begin{aligned}
\vec{v} & =\left(\vec{v} \cdot \overrightarrow{p^{\prime}}\right)[\cos \theta \vec{p}+\sin \theta \vec{q}]+\left(\vec{v} \cdot \overrightarrow{q^{\prime}}\right)[-\sin \theta \vec{p}+\cos \theta \vec{q}]+\left(\vec{v} \cdot \overrightarrow{n^{\prime}}\right) \vec{n} \\
& =\cos \theta\left[\left(\vec{v} \cdot \overrightarrow{p^{\prime}}\right) \vec{p}+\left(\vec{v} \cdot \overrightarrow{q^{\prime}}\right) \vec{q}\right]+\sin \theta\left[\left(\vec{v} \cdot \overrightarrow{p^{\prime}}\right) \vec{q}-\left(\vec{v} \cdot \overrightarrow{q^{\prime}}\right) \vec{p}\right]+\left(\vec{v} \cdot \overrightarrow{n^{\prime}}\right) \vec{n}
\end{aligned}
$$

Podemos reescrever a equação (C.10) como sendo

$$
\left(\vec{v} \cdot \overrightarrow{p^{\prime}}\right) \vec{p}+\left(\vec{v} \cdot \overrightarrow{q^{\prime}}\right) \vec{q}=\vec{v}-\left(\vec{v} \cdot \overrightarrow{n^{\prime}}\right) \vec{n}
$$

podemos ainda reescrever a equação (C.10) de uma outra maneira

$$
\left(\vec{v} \cdot \overrightarrow{p^{\prime}}\right) \vec{q}-\left(\vec{v} \cdot \overrightarrow{q^{\prime}}\right) \vec{p}=(\vec{p} \wedge \vec{q}) \wedge \vec{v}=\vec{n} \wedge \vec{v}
$$

Subistituindo as equações (C.13) e (C.14) na equação (C.12) temos

$$
\begin{aligned}
\vec{v} & =\cos \theta\left[\vec{v}-\left(\vec{v} \cdot \overrightarrow{n^{\prime}}\right) \vec{n}\right]+\sin \theta[\vec{n} \wedge \vec{v}]+\left(\vec{v} \cdot \overrightarrow{n^{\prime}}\right) \vec{n} \\
& =\cos \theta \vec{v}+(1-\cos \theta)\left(\vec{v} \cdot \overrightarrow{n^{\prime}}\right) \vec{n}+\sin \theta[\vec{n} \wedge \vec{v}]
\end{aligned}
$$

então podemos escrever a equação (C.16) como uma soma de matrizes

$$
=\cos \theta\left(\begin{array}{c}
v_{1} \\
v_{2} \\
v_{3}
\end{array}\right)+(1-\cos \theta)\left(\begin{array}{ccc}
n_{1}^{2} & n_{2} n_{1} & n_{3} n_{1} \\
n_{1} n_{2} & n_{2}^{2} & n_{3} n_{2} \\
n_{1} n_{2} & n_{2} n_{3} & n_{3}^{2}
\end{array}\right)\left(\begin{array}{c}
v_{1}^{\prime} \\
v_{2}^{\prime} \\
v_{3}^{\prime}
\end{array}\right)+\sin \theta\left(\begin{array}{c}
n_{2} v_{3}-n_{3} v_{2} \\
n_{3} v_{1}-n_{1} v_{3} \\
n_{1} v_{2}-n_{2} v_{1}
\end{array}\right)
$$

Agora comparando com

$$
\overrightarrow{v^{\prime}}=\mathbf{A} \vec{v}
$$

chegamos a matriz de rotação $\mathbf{A}(\theta)$

$$
\left(\begin{array}{rrr}
\cos \theta+(1+\cos \theta) n_{1}^{2} & (1-\cos \theta) n_{1} n_{2}-n_{3} \sin \theta & (1-\cos \theta) n_{1} n_{3}+n_{2} \sin \theta \\
(1-\cos \theta) n_{1} n_{2}+n_{3} \sin \theta & \cos \theta+(1-\cos \theta) n_{2}^{2} & (1-\cos \theta) n_{2} n_{3}-n_{1} \sin \theta \\
(1-\cos \theta) n_{3} n_{1}-n_{2} \sin \theta & (1-\cos \theta) n_{3} n_{2}+n_{1} \sin \theta & \cos \theta+(1-\cos \theta) n_{3}{ }^{2}
\end{array}\right)
$$




\section{Apêndice D}

\section{Anisotropia do Wobbling-cone}

Dada a expressão para a anisotropia de uma distribuição na esfera de dipolos com movimento restrito a um cone

$r(t)=\iiint(-\sin \theta \sin \phi \sin \alpha+\cos \alpha \cos \theta)^{2} \gamma\left(\alpha, \theta_{0}, \phi_{0}\right) G\left(\theta, \phi, t ; \theta_{0}, \phi_{0}, 0\right) d \Omega_{\hat{n}}(0) d \Omega_{\hat{n}}(t) d \Omega_{L a b}$

onde

$$
\gamma\left(\theta_{0}, \phi_{0}, \alpha\right)=\frac{3}{8 \pi^{2}\left(1-\cos \theta_{\max }\right)}(-\sin \theta \sin \phi \sin \alpha+\cos \alpha \cos \theta)^{2},
$$

vamos explicitar cada um dos termos correspondentes a expressão. Com isso temos

$$
\begin{aligned}
= & \frac{3}{2} \frac{3}{8 \pi^{2}\left(1-\cos \theta_{\text {max }}\right)}\left\{\sum _ { m = 0 } ^ { \infty } \sum _ { n = 1 } ^ { \infty } \left[\int_{0}^{2 \pi} \frac{\cos m \phi(t) \sin ^{2} \phi(t)}{\pi H_{n}^{m} \epsilon} d \phi(t) \int_{0}^{2 \pi} \cos m \phi_{0} \sin ^{2} \phi_{0} d \phi_{0}\right.\right. \\
& \left.+\int_{0}^{2 \pi} \frac{\sin m \phi(t) \sin ^{2} \phi(t)}{\pi H_{n}^{m}} d \phi(t) \int_{0}^{2 \pi} \sin m \phi_{0} \sin ^{2} \phi_{0} d \phi_{0}\right] \int_{0}^{\theta_{\max }} P_{l_{n}^{m}}^{m}(\mu(t)) \sin ^{2} \theta(t) \sin \theta(t) d \theta(t) \\
& \int_{0}^{\theta_{\max }} P_{l_{n}^{m}}^{m}(\mu(0)) \sin ^{2} \theta_{0} \sin \theta_{0} d \theta_{0} \int_{0}^{2 \pi} d \beta \int_{0}^{\pi} \sin ^{4} \alpha \sin \alpha d \alpha \\
& -2\left[\int_{0}^{2 \pi} \frac{\cos m \phi(t) \sin \phi(t)}{\pi H_{n}^{m} \epsilon} d \phi(t) \int_{0}^{2 \pi} \cos m \phi_{0} \sin ^{2} \phi_{0} d \phi_{0}+\int_{0}^{2 \pi} \frac{\sin m \phi(t) \sin \phi(t)}{\pi H_{n}^{m}} d \phi(t)\right. \\
& \left.\cdot \int_{0}^{2 \pi} \sin m \phi_{0} \sin ^{2} \phi_{0} d \phi_{0}\right] \int_{0}^{\theta_{\max }} P_{l_{n}^{m}}^{m}(\mu(t)) \sin ^{2} \theta(t) \cos \theta(t) d \theta(t) \int_{0}^{\theta_{\max }} P_{l_{n}^{m}}^{m}(\mu(0)) \sin ^{3} \theta_{0} d \theta_{0}
\end{aligned}
$$




$$
\begin{aligned}
& \int_{0}^{2 \pi} d \beta \int_{0}^{\pi} \sin ^{4} \alpha \cos \alpha d \alpha \\
& +\left[\int_{0}^{2 \pi} \frac{\cos m \phi(t)}{\pi H_{n}^{m} \epsilon} d \phi(t) \int_{0}^{2 \pi} \cos m \phi_{0} \sin ^{2} \phi_{0} d \phi_{0}+\int_{0}^{2 \pi} \frac{\sin m \phi(t)}{\pi H_{n}^{m}} d \phi(t) \int_{0}^{2 \pi} \sin m \phi_{0} \sin ^{2} \phi_{0} d \phi_{0}\right] \\
& \int_{0}^{\theta_{\max }} P_{l_{n}^{m}}^{m}(\mu(t)) \cos ^{2} \theta(t) \sin \theta(t) d \theta(t) \int_{0}^{\theta_{\max }} P_{l_{n}^{m}}^{m}(\mu(0)) \sin ^{2} \theta_{0} d \theta_{0} \int_{0}^{2 \pi} d \beta \int_{0}^{\pi} \sin ^{3} \alpha \cos ^{2} \alpha d \alpha \\
& -2\left[\int_{0}^{2 \pi} \frac{\cos m \phi(t) \sin ^{2} \phi(t)}{\pi H_{n}^{m} \epsilon} d \phi(t) \int_{0}^{2 \pi} \cos m \phi_{0} \sin \phi_{0} d \phi_{0}+\int_{0}^{2 \pi} \frac{\sin m \phi(t) \sin ^{2} \phi(t)}{\pi H_{n}^{m}} d \phi(t)\right. \\
& \left.\int_{0}^{2 \pi} \sin m \phi_{0} \sin \phi_{0} d \phi_{0}\right] \int_{0}^{\theta_{\max }} P_{l_{n}^{m}}^{m}(\mu(t)) \sin ^{2} \theta(t) \sin \theta(t) d \theta(t) \int_{0}^{\theta_{\max }} P_{l_{n}^{m}}^{m}(\mu(0)) \sin ^{2} \theta_{0} \cos \theta_{0} d \theta_{0} \\
& \int_{0}^{2 \pi} d \beta \int_{0}^{\pi} \sin ^{4} \alpha \cos \alpha d \alpha+4\left[\int_{0}^{2 \pi} \frac{\cos m \phi(t) \sin \phi(t)}{\pi H_{n}^{m} \epsilon} d \phi(t) \int_{0}^{2 \pi} \cos m \phi_{0} \sin \phi_{0} d \phi_{0}\right. \\
& \left.+\int_{0}^{2 \pi} \frac{\sin m \phi(t) \sin \phi(t)}{\pi H_{n}^{m}} d \phi(t) \int_{0}^{2 \pi} \sin m \phi_{0} \sin \phi_{0} d \phi_{0}\right] \int_{0}^{\theta_{\max }} P_{l_{n}^{m}}^{m}(\mu(t)) \sin ^{2} \phi(t) \cos \phi(t) d \phi(t) \\
& \int_{0}^{\theta_{\max }} P_{l_{n}^{m}}^{m}(\mu(0)) \sin ^{2} \phi_{0} \cos \phi_{0} d \phi_{0} \int_{0}^{2 \pi} d \beta \int_{0}^{\pi} \sin ^{3} \alpha \cos ^{2} \alpha d \alpha-2\left[\int_{0}^{2 \pi} \frac{\cos m \phi(t)}{\pi H_{n}^{m} \epsilon} d \phi(t)\right. \\
& \left.\int_{0}^{2 \pi} \cos m \phi_{0} \sin \phi_{0} d \phi_{0}+\int_{0}^{2 \pi} \frac{\sin m \phi(t)}{\pi H_{n}^{m}} d \phi(t) \int_{0}^{2 \pi} \sin m \phi_{0} \sin \phi_{0} d \phi_{0}\right] \\
& \int_{0}^{\theta_{\max }} P_{l_{n}^{m}}^{m}(\mu(t)) \cos \theta(t) \sin \theta(t) d \theta(t) \int_{0}^{\theta_{\max }} P_{l_{n}^{m}}^{m}(\mu(0)) \sin ^{2} \theta_{0} \cos \theta_{0} d \theta_{0} \int_{0}^{2 \pi} d \beta \int_{0}^{\pi} \sin ^{2} \alpha \cos ^{3} \alpha d \alpha \\
& +\left[\int_{0}^{2 \pi} \frac{\cos m \phi(t) \sin ^{2} \phi(t)}{\pi H_{n}^{m} \epsilon} d \phi(t) \int_{0}^{2 \pi} \cos m \phi_{0} d \phi_{0}+\int_{0}^{2 \pi} \frac{\sin m \phi(t) \sin ^{2} \phi(t)}{\pi H_{n}^{m}} d \phi(t) \int_{0}^{2 \pi} \sin m \phi_{0} d \phi_{0}\right] \\
& \int_{0}^{\theta_{\max }} P_{l_{n}^{m}}^{m}(\mu(t)) \sin ^{3} \theta(t) d \theta(t) \int_{0}^{2 \pi} P_{l_{n}^{m}}^{m}(\mu(0)) \cos ^{2} \theta_{0} \sin \theta_{0} d \theta_{0} \int_{0}^{2 \pi} d \beta \int_{0}^{\pi} \sin ^{3} \alpha \cos ^{2} \alpha d \alpha \\
& -2\left[\int_{0}^{2 \pi} \frac{\cos m \phi(t) \sin \phi(t)}{\pi H_{n}^{m} \epsilon} d \phi(t) \int_{0}^{2 \pi} \cos m \phi_{0} d \phi_{0}+\int_{0}^{2 \pi} \frac{\sin m \phi(t) \sin \phi(t)}{\pi H_{n}^{m}} d \phi(t) \int_{0}^{2 \pi} \sin \phi_{0} d \phi_{0}\right] \\
& \int_{0}^{\theta_{\max }} P_{l_{n}^{m}}^{m}(\mu(t)) \sin ^{2} \theta(t) \cos \theta(t) d \theta(t) \int_{0}^{\theta_{\max }} P_{l_{n}^{m}}^{m}(\mu(0)) \cos ^{2} \theta_{0} \sin \theta_{0} d \theta_{0} i n t_{0}^{2 \pi} d \beta \int_{0}^{\pi} \sin ^{2} \alpha \cos ^{3} \alpha d \alpha \\
& +\left[\int_{0}^{2 \pi} \frac{\cos m \phi(t)}{\pi H_{n}^{m} \epsilon} d \phi(t) \int_{0}^{2 \pi} \cos m \phi_{0} d \phi_{0}+\int_{0}^{2 \pi} \frac{\sin m \phi(t)}{\pi H_{n}^{m}} d \phi(t) \int_{0}^{2 \pi} \sin m \phi_{0} d \phi_{0}\right] \\
& \int_{0}^{\theta_{\max }} P_{l_{n}^{m}}^{m}(\mu(t)) \cos ^{2} \theta(t) \sin \theta(t) d \theta(t) \int_{0}^{\theta_{\max }} P_{l_{n}^{m}}^{m}(\mu(0)) \cos ^{2} \theta_{0} \sin \theta_{0} d \theta_{0} \int_{0}^{2 \pi} d \beta \\
& \left.\int_{0}^{\pi} \cos ^{4} \alpha \sin \alpha d \alpha\right\} \exp \left(-l_{n}^{m}\left(l_{n}^{m}+1\right) D_{w} t\right)-\frac{1}{2}
\end{aligned}
$$

A equação (D.3) só pode ser resolvida analiticamente para $m=0$ e $n=1$, o que proporciona $P_{l_{1}^{0}}^{0}=1$ e $l_{1}^{0}=0$, isso para qualquer valor de $\theta_{\max }$. 


$$
\begin{aligned}
r(t \rightarrow \infty)= & \frac{3}{2} \frac{3}{8 \pi^{2}\left(1-\cos \theta_{\max }\right)} \frac{1}{2 \pi H_{1}^{0}}\left\{\int_{0}^{2 \pi} \sin ^{2} \phi(t) d \phi(t) \int_{0}^{2 \pi} \sin ^{2} \phi_{0} d \phi_{0}\right. \\
& \int_{0}^{\theta_{\max }} \sin ^{3} \theta(t) d \theta(t) \int_{0}^{\theta_{\max }} \sin ^{3} \phi_{0} d \phi_{0} \int_{0}^{2 \pi} d \beta \int_{0}^{\pi} \sin ^{5} \alpha d \alpha \\
& \int_{0}^{2 \pi} d \phi(t) \int_{0}^{2 \pi} \sin \phi_{0} d \phi_{0} \int_{0}^{\theta_{\max }} \cos ^{2} \theta(t) \sin \theta(t) d \theta(t) \int_{0}^{\theta_{\max }} \sin ^{3} \theta_{0} d \theta_{0} \\
& \int_{0}^{2 \pi} d \beta \int_{0}^{\pi} \sin ^{3} \alpha \cos ^{2} \alpha d \alpha+\int_{0}^{2 \pi} \sin \phi(t) d \phi(t) \int_{0}^{2 \pi} d \phi_{0} \int_{0}^{\theta_{\max }} \sin ^{2} \theta(t) d \theta(t) \\
& \int_{0}^{\theta_{\max }} \cos ^{2} \theta_{0} \sin \theta_{0} d \theta_{0} \int_{0}^{2 \pi} d \beta \int_{0}^{\pi} \sin ^{2} \alpha \cos ^{2} \alpha d \alpha+\int_{0}^{2 \pi} d \phi(t) \int_{0}^{2 \pi} d \phi_{0} \\
& \int_{0}^{\theta_{\max }} \cos ^{2} \theta(t) \sin \theta(t) d \theta(t) \int_{0}^{\theta_{\max }} \cos ^{2} \theta_{0} \sin \theta_{0} d \theta_{0} \\
& \left.\int_{0}^{2 \pi} d \beta \int_{0}^{2 \pi} \cos ^{4} \alpha \sin \alpha d \alpha\right\}+\frac{1}{2}
\end{aligned}
$$

resolvendo cada uma das integrais temos

$$
r(t \rightarrow \infty)=\frac{2}{5}\left[\frac{1}{2} \cos \theta_{\max }\left(1+\cos \theta_{\max }\right)\right]^{2}
$$




\section{Apêndice E}

\section{Trigonometria esférica}

Dada uma esfera de raio arbitrário, se um plano passar pelo centro da esfera, irá dividila em dois hemisférios idênticos. A circunferência interceptada pelo plano é a circunferência máxima. Quando duas circunferências máximas se interceptam em um ponto, na superfície da esfera, forma entre si um ângulo chamado de esférico. A medida do ângulo esférico é igual a medida do ângulo plano, formado entre duas retas tangentes aos dois arcos e contida no mesmo plano de cada uma das circunferências máximas.

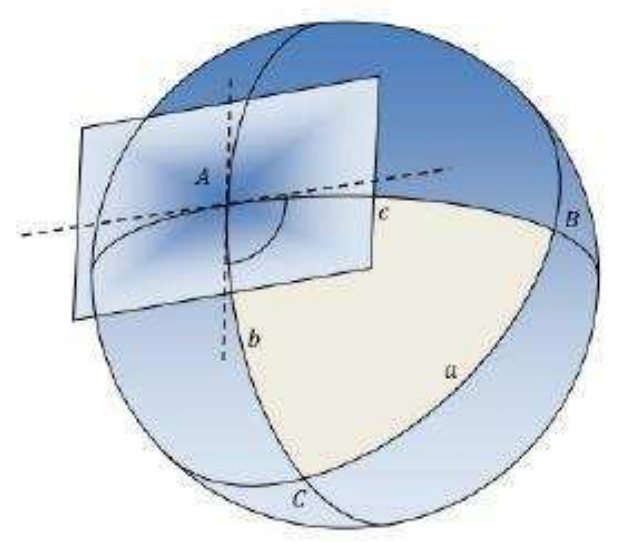

Figura E.1: Retas tangentes a esferas máximas.

Se tivermos três circunferências máximas na esfera, teremos três ângulos esféricos e consequentemente um triângulo esférico. Os lados do triângulo esférico são arcos esféricos, cuja medida é o ângulo correspondente entre as extremidades do lado do triângulo e o centro da circunferência máximas. A figura E.2, mostra uma esfera com três circunferências máximas, 
os ângulos esféricos $(A, B, C)$ formados com a interceptação das circunferência máxima, o triângulo esférico e os arcos esféricos $(a, b, c)$.
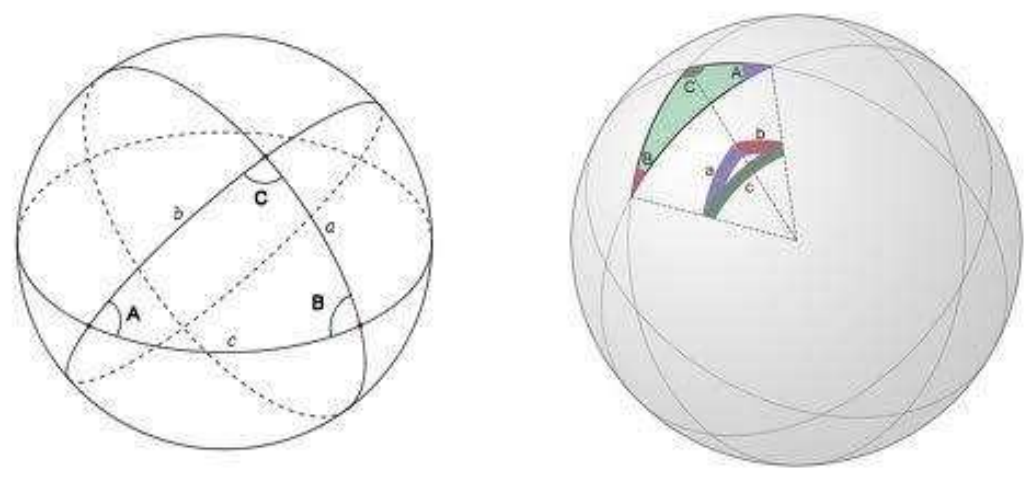

Figura E.2

No triângulo esférico mostrado na figura (E.2), o lado a mede o ângulo $B O C$, o lado $b$ mede o ângulo $A O C$ e o lado $c$ mede o ângulo $A O B$. Se desejarmos, por exemplo, calcular o arco esférico $a$, traçamos uma reta tangente em $A$, a reta deve estar no plano da circunferência máxima, que está contido os pontos $A$ e $B$. Uma outra reta tangente no ponto, a reta deve estar no plano da circunferência máxima, que está contido os pontos $A$ e $C$, figura (E.3).

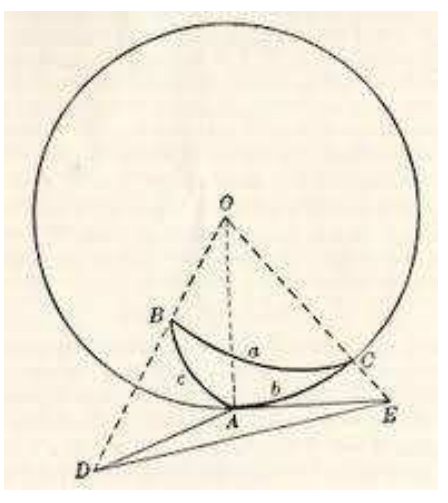

Figura E.3

A reta $O A$ é perpendicular às duas retas tangentes no ponto A. Prolongando a reta $O B$, até interceptar a reta tangente em um ponto $D$, temos o triângulo retângulo $D O A$, figura (E.4), cujas relações trigonométricas são

$$
\begin{aligned}
\tan c=\frac{A D}{O A} & =\frac{\sin c}{\cos c}, \quad A D \cos c=O A \sin c \\
\cos c=\frac{O A}{O D}, \quad O A & =O D \cos c, \quad O D=O A \sec c .
\end{aligned}
$$

Prolongando a reta OC, até interceptar a outra reta tangente no ponto E, temos o 


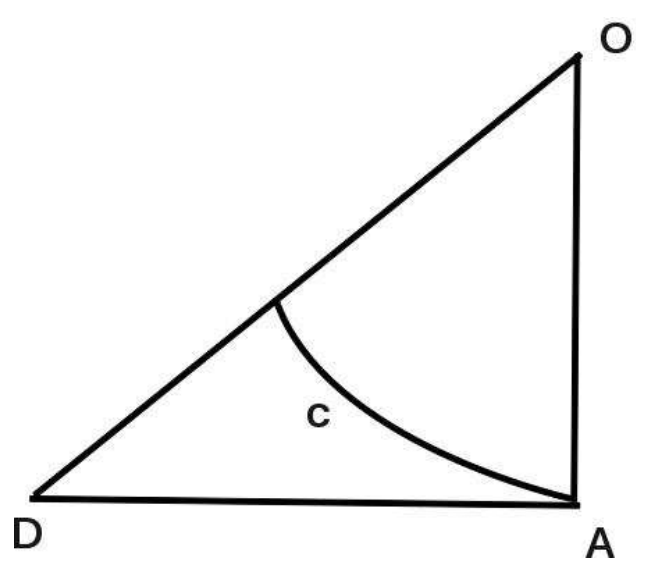

Figura E.4: Triângulo DOA

triângulo AOE, figura (E.5), cujas relações trigonométricas são

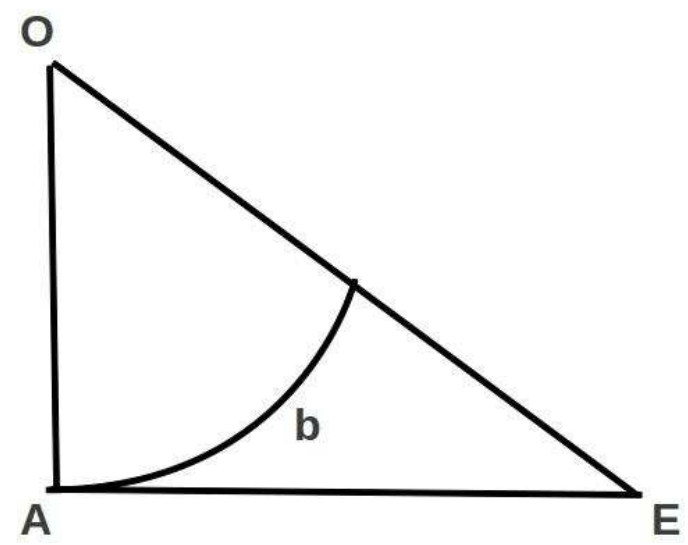

Figura E.5: Triângulo $A O E$

$$
\begin{array}{r}
\tan b=\frac{A E}{O A}=\frac{\sin b}{\cos b}, \quad A E \cos c=O A \sin b \\
\cos b=\frac{O A}{O E}, \quad O A=O E \cos b, \quad O E=O A \sec b
\end{array}
$$

Observando a figura (E.3), temos outros dois triângulos, o triângulo DOE e o triângulo DAE. O triângulo DOE, figura (E.6), através da lei dos cosseno, proporciona a seguinte expressão

$$
D E^{2}=O D^{2}+A E^{2}-2(D A)(O E) \cos a
$$

e o triângulo DAE, figura (E.7), também através da lei dos cossenos, fornece a expressão

$$
D E^{2}=D A^{2}+A E^{2}-2(D A)(A E) \cos A .
$$




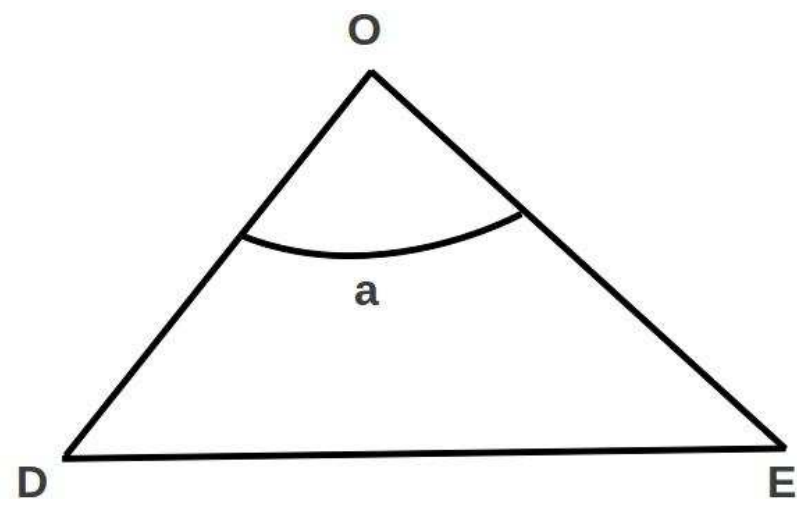

Figura E.6: Triângulo DOE

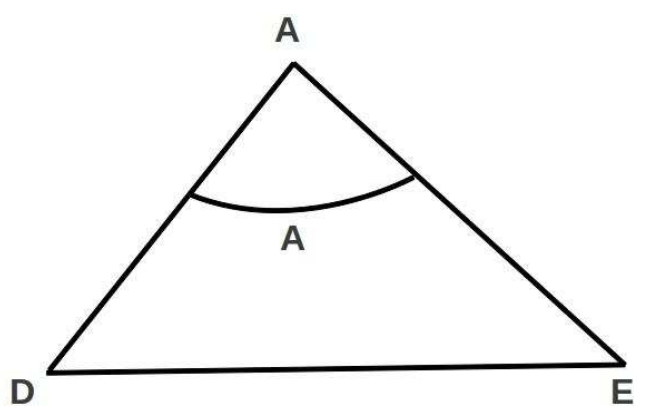

Figura E.7: Triãngulo DAE

Substituindo as equações (E.1), (E.2) em (E.3) temos

$$
D E^{2}=O A^{2}\left[\tan ^{2} c+\tan ^{2} b-2 \tan c \tan b \cos A\right] .
$$

Substituindo as equações (E.1), (E.2) em (E.4) temos

$$
D E^{2}=O A^{2}\left[\sec ^{2} c+\sec ^{2} b-2 \sec b \sec c \cos a\right] .
$$

igualando a equação (E.5) com a equação (E.6) temos que

$$
\sec ^{2} c+\sec ^{2} b-2 \sec b \sec c \cos a=\tan 2 c+\tan 2 b-2 \tan b \tan c \cos A
$$

Das relações trigonométricas fundamentais em triângulos planos temos que

$$
\sec ^{2} c=1+\tan ^{2} c \quad \sec ^{2} b=1+\tan ^{2} b
$$


Obtemos

$$
\cos a=\cos b \cos c+\sin b \sin c \cos A
$$

O mesmo raciocínio é feito para calcular os outros arcos esféricos $b$ e $c$

$$
\begin{aligned}
& \cos b=\cos a \cos c+\sin a \sin c \cos B \\
& \cos c=\cos a \cos b+\sin a \sin b \cos C .
\end{aligned}
$$


104 APÊNDICE E 


\section{Referências Bibliográficas}

[1] J. R. Lakowicz, Principles of Fluorescence Spectroscopy. Plenum Press, 1983. 1, 13, 14

[2] R. F. Turchiello, M. T. Lamy-Freund, I. Y. Hirata, L. Juliano, and A. S. Ito, "Orthoaminobenzoic acid as a fluorescent probe for the interaction between peptides and micelles," Biophysical chemistry, vol. 73, pp. 217-225, 1998. 2

[3] R. F. Turchiello, M. T. Lamy-Freund, I. Y. Hirata, L. Juliano, and A. S. Ito, "Orthoaminobenzoic acid-labeled bradykinins in interaction with lipid visicle: fluorescence study.," Biopolymers, vol. 65, pp. 338-346, 2002. 2

[4] L. R. Montaldi, M. Berardi, E. S. Souza, L. Juliano, and A. S. Ito, "End-to-end distance distribution in fluorescent derivatve of bradykinin in interaction with lipid vesicles," $J$. of fluorescence, vol. 22, pp. 1151-1158, 2012. 2

[5] M. T. Lamy-Freund and K. A. Riske, "The peculiar thermo-structural behavior of the anionic lipid dmpg.," Chemistry and Physics of lipids, vol. 1, pp. 19-32, 2003. 2

[6] W. M. Pazin, "Anisotropia de fluorescencia: aplicacao em membranas modelos," Master's thesis, Universidade de SÃ£o Paulo - USP - FFCLRP, 2012. 2

[7] K. Kinosita, S. Kawato, and D. A. Ikegami, "A theory of fluorescence polarization decay in membrane," Biophysical j., vol. 20, pp. 289-305, 1977. 2, 14

[8] M. Krishna, R. Das, N. Periasamy, and R. Nityananda, "Translational diffusion of fluorescent probes on spherical: Monte carlo simulations, theory and fluorescence anisotropy experiment," J. Chem. Phys., vol. 112, pp. 8502-8514, 2000. 2, 62 
[9] M. Krishna, A. Srivastava, and N. Periasamy, "Rotational dynamics of surface probes in lipid vesicles," Biophysical Chemistry, vol. 90, pp. 123-133, 2001. 2, 62

[10] J. Garcia de La Torres and C. L. Martinez, "Brownian dynamics simualation of restricted rotational diffusion," Biophys. J., vol. 52, pp. 303-310, 1987. 2

[11] B. Valeur, Molecular Fluorescence: principles and applications. Wiley-VCH, 2002. 5

[12] P. A. van Paridon, J. K. Shute, K. W. A. Wirtz, and A. J. W. G. Visser, "A fluorescence decay study of parinaroyl-phosphatidylinositol incorpored into artificial and natural membranes," Eur. Biophys. J., vol. 16, pp. 53-63, 1988. 9

[13] S. Kawato and K. K. J. A. Ikegami, "Dynamic structure of lipid bilayers studies by nanosecond fluorescence techniques," Biochemistry, vol. 16, pp. 2319-2324, 1977. 13, 14

[14] J. R. Lakowicz, I. Gryczynski, and E. Dnielsen, "Anomalous differential polarized phase angles for two-photon excitation with isotropic depolarizing rotations," Chemical Physics Letters, vol. 191, pp. 47-53, 1992. 14

[15] U. van der Heide, G. van Ginkel, and Y. Levine, "Dph is localised in two distinct population in lipid vesicle," Chemical Physics Letters, vol. 253, pp. 118-122, 1996. 14, 74

[16] E. Pebay-Peyroula, E. J. Dufourc, and A. G. Szabo, "Location of diphenyl-hexatriene and trimethylammonium-diphenyl-hexatriene in dipalmitoylphosphatidylcholine bilayer by neutron diffraction," Biophysical chemistry, vol. 53, pp. 45-56, 1994. 14, 15, 74

[17] I. Konopasek, J. Vecer, K. Strzalka, and E. Amler, "Short-lived fluorescence component of dph reports on lipid-water interface of biological membranes," chemistry and physics of lipids, vol. 130, pp. 135-144, 2004. 15

[18] H. J. Arfken, George B Weber, Mathematical methods for physicists. San Diego Academic Press, 4a ed., 1995. 20 
[19] C. Wang and R. Pecora, "Time-correlation function for restricted rotationl diffusional," J. Chem. Phys., vol. 72(10), pp. 533-5340, 1980. 29

[20] H. F. Bauer, "Tables of the roots of the associated legendre function with respect to the degree," Mathematics of computation, vol. 46, pp. 61-62, 1986. 45

[21] H. F. Bauer, "Tables of the roots of the associated legendre function with respect to the degree," Mathematics of computation, vol. 46(174), pp. S29-S41, 1986. 45

[22] W. H. Press, S. A. Teukolsky, W. T. Vetterling, and B. P. Flannery, Numerical Recipes in $C$; The art of scientific computing. Cambridge University Press, Cambridge, 2nd ed., 1992. 53

[23] P. R. Bevington and D. K. Robinson, Data Reduction and Error Analysis for the Physical Sciences. McGraw-hill, New York, 2nd ed., 1994. 53 